University of Rhode Island

DigitalCommons@URI

Open Access Dissertations

2019

\title{
ULTRAFAST COHERENT SPECTROSCOPIES AND LASER SOURCES BASED ON THIRD-ORDER NONLINEAR OPTICAL PHENOMENA
}

Adam D. Card

University of Rhode Island, card.adam@gmail.com

Follow this and additional works at: https://digitalcommons.uri.edu/oa_diss

\section{Recommended Citation}

Card, Adam D., "ULTRAFAST COHERENT SPECTROSCOPIES AND LASER SOURCES BASED ON THIRDORDER NONLINEAR OPTICAL PHENOMENA" (2019). Open Access Dissertations. Paper 876. https://digitalcommons.uri.edu/oa_diss/876

This Dissertation is brought to you for free and open access by DigitalCommons@URI. It has been accepted for inclusion in Open Access Dissertations by an authorized administrator of DigitalCommons@URI. For more information, please contact digitalcommons-group@uri.edu. 
ULTRAFAST COHERENT SPECTROSCOPIES AND LASER SOURCES BASED ON THIRD-ORDER NONLINEAR OPTICAL PHENOMENA BY

ADAM D CARD

A DISSERTATION SUBMITTED IN PARTIAL FULFILLMENT OF THE REQUIREMENTS FOR THE DEGREE OF DOCTOR OF PHILOSOPHY IN PHYSICS

UNIVERSITY OF RHODE ISLAND 2019 


\title{
DOCTOR OF PHILOSOPHY DISSERTATION \\ OF
}

\author{
ADAM D CARD
}

\section{APPROVED:}

Dissertation Committee:

Major Professor Feruz Ganikhanov

David Heskett

Tao Wei

Gerhard Muller

Nasser H. Zawia

DEAN OF THE GRADUATE SCHOOL

UNIVERSITY OF RHODE ISLAND

2019 


\begin{abstract}
This document is composed of four different research efforts. In the first chapter of this dissertation, I present my experimental work on seeded Yb-doped Q-switched fiber laser based on stimulated Brillouin scattering (SBS). I began by experimenting with different types of fiber laser cavity configurations in order to find an ideal cavity that would successfully generate the free-running stochastic nonlinear SBS mirror as well as to reinforce the injected light mode. Experimentally, the ideal configuration involved a diffraction grating as the cavity back-mirror in the Littrow configuration with added cavity losses to enhance the SBS effect. Single SBS pulses exhibited the cascaded SBS effect and pulse width (FWHM) typically less than 2 ns. Continuous sine wave modulated light, continuous triangular wave modulated light and a dynamic pulsed halfsine modulated light injections successfully synchronized the SBS repetition rate, and to some degree the pulse-to-pulse intensity, to the waveform frequency. The injected waveforms effectively seeded the SBS Q-switching mechanism and stabilized the laser repetition rate in a novel way. Output spectrum showed the injected waveforms nearly, but not completely, suppressed the free-running cavity modes due to fiber terminations and the output pulse wavelength was slightly blue-shifted compared to the injection spectrum. This was a curious result and is under further investigation.

In the second part of this work, I show experimentation regarding the resolution of fine spectral features within several Raman active vibrational modes in potassium titanyl phosphate (KTP) crystal. Measurements are performed using a femtosecond timedomain coherent anti-Stokes Raman scattering spectroscopy technique that is capable of
\end{abstract}


delivering equivalent spectral resolution of $0.1 \mathrm{~cm}^{-1}$. The Raman spectra retrieved from my measurements show several spectral components corresponding to vibrations of different symmetry with distinctly different damping rates. In particular, linewidths for unassigned optical phonon mode triplet centered at around $820 \mathrm{~cm}^{-1}$ are found to be $7.5 \pm 0.2 \mathrm{~cm}^{-1}, 9.1 \pm 0.3 \mathrm{~cm}^{-1}$, and $11.2 \pm 0.3 \mathrm{~cm}^{-1}$. Results of these experiments will ultimately help to design an all-solid-state source for sub-optical-wavelength waveform generation that is based on stimulated Raman scattering.

In the third section of this document, I demonstrate and analyze a series of experiments in traditional and soft condensed matter using coherent optical spectroscopy and microscopy with ultrafast time resolution. I will show capabilities of resolving both real and imaginary parts of the third-order nonlinearity in the vicinity of Raman resonances from a medium probed within microscopic volumes with an equivalent spectral resolution of better than $0.1 \mathrm{~cm}^{-1}$. I can differentiate between vibrations of various types within unit cells of crystals, as well as perform targeted probes of areas within biological tissue. Vibrations within TiO6 octahedron and the ones for the Ti-O-P intergroup were studied in potassium titanyl phosphate crystal to reveal multiline structure within targeted phonon modes with closely spaced vibrations having distinctly different damping rates ( $\sim 0.5 \mathrm{ps}-1$ versus $\sim 1.1 \mathrm{ps}-1)$. I also detected 1.7-2.6 ps-1 decay of C-C stretching vibrations in fat tissue and compared that with the corresponding vibration in oil.

I lastly demonstrate an effective microspectroscopy technique by tracing the dispersion of second order nonlinear susceptibility $\left(\chi^{(2)}\right)$ in a monolayer tungsten diselenide $\left(\mathrm{WSe}_{2}\right)$. The $\chi^{(2)}$ dispersion obtained with better than $3 \mathrm{meV}$ photon energy 
resolution showed peak value being within $6.3-8.4 \times 10^{-19} \mathrm{~m}^{2} / \mathrm{V}$ range. I estimate the fundamental bandgap to be at $2.2 \mathrm{eV}$. Sub-structure in the $\chi^{(2)}$ dispersion reveals a contribution to the nonlinearity due to exciton transitions with exciton binding energy estimated to be at $0.7 \mathrm{eV}$. 


\section{ACKNOWLEDGEMENTS}

I would like to express my sincere gratitude to the University of Rhode Island for giving me the opportunity to continue my education and allowing me to fulfill my dream of attaining my doctorate in physics. I would also like to thank the Department of Physics and its staff for its ongoing support, without which attaining this doctorate would not be possible.

I would like to thank my advisor, Dr. Feruz Ganikhanov, for his support and guidance throughout my research efforts and subsequent publications. If it had not been for his immense support, patience and guidance, this thesis would not have been possible. It has been a great privilege to work and learn under Dr. Ganikhanov's tutelage.

I would like to thank my graduate committee members, Dr. Heskett, Dr. Wei, Dr. Hayes, and Dr. Muller, for their continued effort, patience and support throughout this process.

I also would like to thank my fellow graduate students of the Department of Physics for their support, patience and kindness during my five years here at the University of Rhode Island.

Lastly, I would like to take the opportunity to thank my parents, my sister and my loving wife, Jennifer. Without their love, wisdom, patience, encouragement and unceasing support this would not have been possible. I cannot describe how grateful I am to have them in my life. 


\section{PREFACE}

The goal of this work is to develop and construct a novel Q-switched fiber laser based upon stimulated Brillouin scattering (SBS) as a Q-switching mechanism, to apply ultrafast coherent spectroscopy techniques based upon third-order nonlinear optical phenomena and to study second-harmonic generation of an atomically thin crystal sample. All work presented in the body of this document was performed at the University of Rhode Island.

This dissertation is written in the 'Manuscript Format' using the Thesis/Dissertation template of the University of Rhode Island. This document will include four chapters. Each chapter corresponds to a manuscript that has been previously published, accepted for publication or soon to be submitted. The figures of each manuscript are listed under the corresponding chapter on the list of figures. Two appendices have been included for ancillary information on the background of the work presented and the experimental methods used in all contained research efforts.

The results of our studies presented in this document were published or are soon to be published in four papers:

1. A. Card and F. Ganikhanov, "Seeded Yb-doped Q-switched fiber laser based on stimulated Brillouin scattering (SBS)," OSA Continuum. Soon to be submitted.

2. A. Card, M. Mokim and F. Ganikhanov, "Resolving fine spectral features in lattice vibrational modes using femtosecond coherent spectroscopy," AIP Advances 6, 025115 (2016). 
3. A. Card, M. Mokim, F. Ganikhanov, "Quantitative ultrafast spectroscopy and microscopy of traditional and soft condensed matter," Applied Sciences 8, 1317 (2018).

4. M. Mokim, A. Card, B. Sah, F. Ganikhanov, "Dispersion of the resonant second order nonlinearity in 2D semiconductors probed by femtosecond continuum pulses," AIP Advances 7, 105121 (2017). 


\section{TABLE OF CONTENTS}

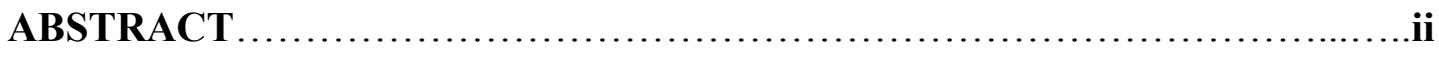

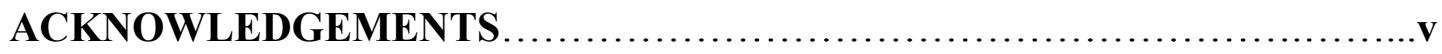

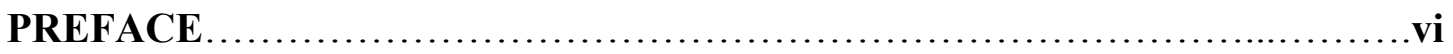

TABLE OF CONTENTS ................................................

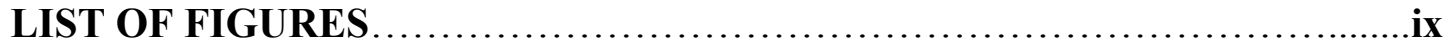

LIST OF ACRONYMS ............................................

CHAPTER 1. Seeded Yb-doped Q-switched fiber laser based on stimulated Brillouin

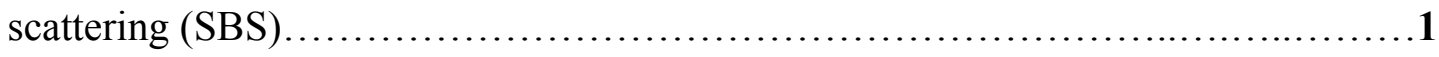

CHAPTER 2. Resolving fine spectral features in lattice vibrational modes using

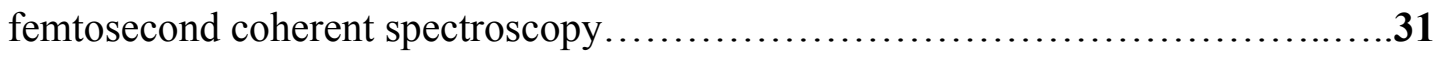

CHAPTER 3. Quantitative ultrafast spectroscopy and microscopy of traditional and

soft condensed matter......................................................

CHAPTER 4. Dispersion of the resonant second order nonlinearity in 2D

semiconductors probed by femtosecond continuum pulses...................80

APPENDIX A. Sources of light and supercontinuum generation................94

APPENDIX B. Experimental techniques and procedures....................100

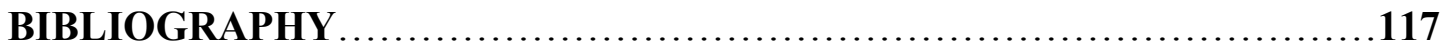




\section{LIST OF FIGURES}

FIGURE

PAGE

Figure 1.1. Theoretical interpretation of study: (a) $1064 \mathrm{~nm}$ waveform (orange) is injected into inverted fiber and is amplified to SBS threshold as it passes through the fiber generating the nonlinear SBS mirror. Acoustic sound wave is in grey and Stokes light is in red; (b) The cascaded SBS effect is shown where multiple Stokes orders exist creating acoustic sound waves (grey) at either end of the double-clad fiber

Figure 1.2. Experimental setup. MZ: Mach-Zehnder; ISO: isolator; VA: variable attenuator; PM: power meter; OSA: optical spectrum analyzer; BS: 50/50 beamsplitter; LD: laser diode; DM: dichroic mirror; AL: aspheric lens; TM: transverse magnetic......21 Figure 1.3. Oscilloscope traces of free-running SBS with HR mirror configuration with varying pumpo power. (a) Pump power $433 \mathrm{~mW}$ (b) Pump power 1.08W (c) Pump power

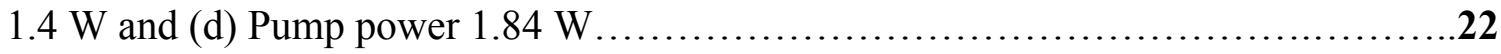
Figure 1.4. Output measurements for the HR mirror cavity configuration: (a) Output power as a function of pump power (b) Output spectrum for low pump power $433 \mathrm{~mW}$ (c) Output spectrum for high pump power $1.84 \mathrm{~W}$

Figure 1.5. Oscilloscope traces of free-running SBS with bare-fiber cavity configuration with varying pump power. (a) Pump power $762 \mathrm{~mW}$ (b) Pump power $1.08 \mathrm{~W}$ (c) Pump power $1.4 \mathrm{~W}$ (d) Pump power $2.03 \mathrm{~W}$.

Figure 1.6. Output measurements for the free-running SBS lasing with the bare-fiber cavity configuration. (a) Output power as a function of pump power (b) Spectral output for pump power $954 \mathrm{~mW}$ (c) Spectral output for pump power $1.72 \mathrm{~W}$ 
Figure 1.7. (a) Injection spectrum (b) ASE spectrum slightly above threshold, $1064 \mathrm{~nm}$ (dotted red), $1072 \mathrm{~nm}$ (dotted blue).

Figure 1.8. Output laser spectra with $\mathrm{CW}$ injection and partial mirror with varying losses:

(a) maximum loss (b) moderate loss (c) minimum loss .24

Figure 1.9. Oscilloscope traces in the Littrow configuration: (a) Pump power $\sim 824 \mathrm{~mW}$

(b) Pump power $\sim 1.08 \mathrm{~W}$ (c) Pump power $\sim 1.47 \mathrm{~W}$ (d) Pump power $\sim 1.84 \mathrm{~W}$. .25

Figure 1.10. Output measurements for the Littrow configuration configuration: (a) Output power as a function of pump power (b) Output spectrum wide span at pump power $2.1 \mathrm{~W}$ (c) Output spectrum narrow span at pump power $2.1 \mathrm{~W}$. .25

Figure 1.11. Oscilloscope traces for Littrow configuration with cavity losses: (a) Pump power $\sim 824 \mathrm{~mW}$ (b) Pump power $\sim 1.08 \mathrm{~W}$ (c) Pump power $\sim 1.47 \mathrm{~W}$ (d) Pump power $\sim 1.84 \mathrm{~W}$

Figure 1.12. Output measurements for the free-running SBS with grating with cavity losses. (a) Output power as a function of pump power (b) (c) Output spectra for pump greater than threshold

Figure 1.13. Details of the SBS single-pulse shape without injection: (a) (b) Bare-fiber termination configuration (c) (d) Littrow configuration with loss....

Figure 1.14. Oscilloscope traces for sine wave modulated light injection. (a) (b) Typical output pulse train sine wave injection (blue) and SBS pulses (yellow). (c) (d) Individual SBS pulses with sine wave injection .27

Figure 1.15. Output measurements for continuous sine wave modulated light injection. (a) Output power as a function of pump power (b) (c) Output spectrum (blue) injection spectrum (dotted red) 
Figure 1.16. Oscilloscope traces for the continuous triangular wave modulated light injection. (a) (b) Typical pulse train. (c) (d) Single SBS pulse with injection............28

Figure 1.17. Output measurements for the triangular wave modulated light injection. (a) Output power as a function of pump power (b) (c) Output spectrum (blue) and injection spectrum (dotted red)

Figure 1.18. Oscilloscope traces for half-sine modulated light injection. (a) (b) Typical pulse train with injection (blue) and SBS pulses (yellow) (c) (d) Individual SBS

pulses

Figure 1.19. Output measurements for the half-sine pulse modulated light injection. (a) Output power as a function of pump power (b) (c) Output spectrum (blue) and injection spectrum (dotted red)

Figure 2.1. Experimental diagram and layout for three-color time-domain CARS spectroscopy experiments that employ tunable optical parametric oscillators. Pair of transform-limited $110-150$ fs pulses at $\lambda_{1}$ and $\lambda_{2}$ wavelengths are used to coherently drive Raman active vibrational modes in the vicinity of corresponding $\omega_{1}-\omega_{2}$ frequency shifts. A pulse at $\lambda_{\mathrm{pr}}=765 \mathrm{~nm}$ is time delayed and probes the resulting coherent excitation at different delay times. CARS transients at anti-Stokes frequency are detected within five orders of magnitude. Polarizations of all the three beams were made parallel to each other and aligned within XY-plane of the KTP crystal under study .45

Figure 2.2. (a) Time-domain CARS system instrument response obtained in quartz glass (black curve). Simulated CARS transient for Raman active vibrational mode with dephasing time $T_{2}=150$ fs (red curve). CARS transient detected in KTP crystal under the conditions specified below (blue curve). The broadband OPO pulses were tuned to 1020 
$\mathrm{nm}$ and $1100 \mathrm{~nm}$ center wavelengths to coherently drive the main $v_{l}\left(A_{l g}\right)$ high frequency

Raman active mode at $\sim 700 \mathrm{~cm}^{-1}$. (b) CARS signal spectrum ( ${ }^{S_{a s}(\omega)}$ ) showing a narrower spectral feature on the background of a broadband pedestal corresponding to the transient signal's fast rise time. (c) Time-domain response function $(G(t))$ that was obtained by solving Equations (1) and (2) using Fourier transform method. (d) Dispersion of real (red open circles) and imaginary (blue open circles) parts of the resonant third order optical nonlinearity $\left(\chi^{(3)}(\omega)\right.$ ) in the vicinity of the coherently driven Raman active modes. The part corresponding to Raman spectra (i.e. $\operatorname{Im}\left(\chi^{(3)}(\omega)\right.$ ) is also shown fitted with solid black curve representing a sum of three Lorentz-shaped envelopes with bandwidths of $21 \mathrm{~cm}^{-1}, 17 \mathrm{~cm}^{-1}$, and $25 \mathrm{~cm}^{-1}$ starting from the red shifted $v_{2}\left(E_{g}\right)$-mode (cyan color peak)

Figure 2.3. (a) CARS transient (blue circles) obtained from KTP crystal when unassigned Raman active vibrational modes at $\sim 820 \mathrm{~cm}^{-1}$ are coherently driven and probed. A proper wavelength combination was chosen for the OPOs $\left(\lambda_{1}=967 \mathrm{~nm}\right.$ and $\lambda_{2}=1051 \mathrm{~nm}$ ) so that the corresponding shift is targeted at its center. Simulated CARS signal (black curve) obtained under amplitude, damping rate, spectral shift parameter values for the vibrational modes using retrieved Raman spectra data. (b) Retrieved Raman spectra (blue curve), simulated Lorentz-shape curves for the three spectral components that yield in cumulative fitting curve (black). (c) Corresponding real part of the third order optical nonlinearity .............................................48

Figure 3.1. (a) Experimental set up. The three-color pulses at $\omega_{1}, \omega_{2}, \omega_{3}$ are provided from lab built tunable femtosecond optical parametric oscillator $\left(\mathrm{OPO}_{1,2}\right)$ sources and 
fixed wavelength Ti:sapphire oscillator (Coherent Mira) respectively. GR- diffraction grating 1200 1/mm, BP-bandpass filter, CL-collimating lens objective. Galvo-scanner is from Cambridge Technology, Inc. (model \#6210), photomultiplier tube (PMT) is from Hamamatsu (model \#H9305), data acquisition card (DAQ) is from National Instruments (model 6201); (b) $\mathrm{OPO}_{1,2}$ spectra for nearly transform limited $\mathrm{E}_{1,2}$ pulses used for CARS experiments; (c) Time-domain CARS signal (open circles) obtained from microscope glass when the OPO wavelengths were set to $967 \mathrm{~nm}$ and $1152 \mathrm{~nm}$. The transient signal represents instrument function that allows to measure dephasing times $\left(T_{2}\right)$ shorter than 150 fs. Solid curves are simulated CARS signals that make use Equations (3) and (4) and assuming single exponential decay for $G(t)$ with $150 \mathrm{fs}$ (black) and $250 \mathrm{fs}$ (red) dephasing times.

Figure 3.2. (a) Time-domain CARS signal (open circles) obtained from KTP crystal when the OPO wavelengths were set to $1019 \mathrm{~nm}$ and $1098 \mathrm{~nm}\left(\sim 706 \mathrm{~cm}^{-1}\right)$. Red solid line represents theoretical fit that assumes single exponential decay for $G(t)$ with the dephasing time of 495 fs; (b) Time-domain CARS signal (open circles) obtained from KTP crystal when the OPO wavelengths were set to $967 \mathrm{~nm}$ and $1041 \mathrm{~nm}$. Solid line represents theoretical fit that assumes Raman doublet with $62 \mathrm{~cm}^{-1}$ frequency difference and decaying with the dephasing times of 495 fs and $515 \mathrm{fs}$; (c, d) Corresponding spectra of the resonant third order nonlinearity obtained with the help of the formalism presented in the text. Real part of $\chi^{(3)}$ is shown in black open circles while the imaginary part is shown in blue circles. Solid lines show results of simulations for both parts of the nonlinearity assuming Lorentzian profile for the two components with parameters




Figure 3.3. (a) CARS transients (open circles) obtained from KTP crystal when the OPO wavelengths were set to $967 \mathrm{~nm}$ and $1052 \mathrm{~nm}\left(\sim 830 \mathrm{~cm}^{-1}\right.$ vibration). Solid line represents theoretical fit that assumes three spectral components and is obtained using software algorithm that varies multiple parameters for each component (amplitude, spectral position, dephasing time) variation that assumes Raman doublet with $62 \mathrm{~cm}^{-1}$ frequency difference and decaying with the dephasing times of $950 \mathrm{fs}$ and $1160 \mathrm{fs}$; (b) Corresponding spectrum of the resonant third order nonlinearity $\left(\chi^{(3)}(\omega)\right)$ obtained using experimental CARS transient and applying Equation (5). Imaginary part of $\chi^{(3)}$ is shown in blue open circles. Solid black line is the best fit to the spectrum assuming three components shown in separate colors and with parameters provided in the text; (c) Real

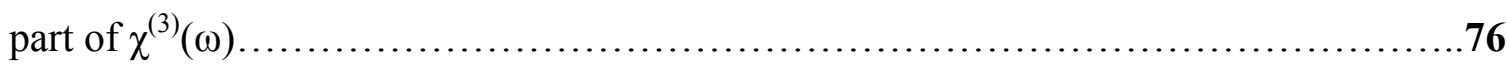
Figure 3.4. (a) CARS transients (open circles) measured in olive oil while vibrations at $\approx 1072 \mathrm{~cm}^{-1}$ were driven and probed. The best fit to the data is given by solid red line for which Equations (3)-(4) have been employed. Corresponding line component parameters are listed in the text; (b) Real (black circles) and imaginary (blue circles) parts of $\chi^{(3)}(\omega)$ obtained using experimental CARS transient and Equation (5) $\ldots \ldots \ldots \ldots \ldots \ldots \ldots \ldots \ldots 77$

Figure 3.5. (a) CARS image obtained from the mouse dermis area at zero time delay between the driving $E_{1}, E_{2}$ pulsed fields and the probe field $E_{3}$. Vibration at $1072 \mathrm{~cm}^{-1}$ has been targeted; (b) CARS image the same area when the probe pulse was delayed by 600 fs; (c) Time-domain CARS signal obtained from the $\sim 20 \mu \mathrm{m}$ diameter fat area located at the center of the mouse tissue for the image shown in Fig. 3.5(a). Red line represents the best fit to the data obtained by using formulae (3)-(4) and varying the corresponding line parameters (see text); (d) Real (black circles) and imaginary (blue circles) parts of $\chi^{(3)}(\omega)$ 
obtained using the experimental CARS transient shown in part (c) and Equation (5). Red line is theoretical fit that makes use two-component Lorentzian function $\left(\Delta v_{1,2}=4.1,6.2\right.$ $\mathrm{cm}^{-1}, 28 \mathrm{~cm}^{-1}$ separation between components, $\left.\mathrm{A}_{1} / \mathrm{A}_{2}=13: 4\right) \ldots \ldots \ldots \ldots \ldots \ldots \ldots \ldots \ldots . . . \ldots 8$ Figure 4.1. (a) Photon diagram SHG process in monolayer semiconductor. (b) Setup: ISO-optical isolator, PCF - photonic crystal fiber, DCM- dichroic mirror, OBJ-high numerical aperture objective lens, SPF-short pass filter. Broadband near-IR pulse derived from femtosecond continuum serves as fundamental beam. Spectra of the generated second harmonic pulses carrying resonant features are analyzed. (c) Fundamental



Figure 4.2. (a) SHG image of a single layer $\mathrm{WSe}_{2}$ flake. Lower left corner of the sample contains three $\mathrm{WSe}_{2}$ layers and therefore show four times smaller signal. (b) Photoluminescence data obtained from the flake when it was excited by $532 \mathrm{~nm}$ continuous wave beam, (c) detected second harmonic beam power versus power of the fundamental beam, (d) SHG signal change across the image along the dashed line

Figure 4.3. (a) Second harmonic signal spectrum $\left(P_{2}^{2 D}\right)$ detected from monolayer part of $\mathrm{WSe}_{2}$ sample (blue), relative transmission $\left({ }^{T_{\omega}}\right)$ of the optical system versus wavelength (cyan), calculated enhancement factor ${ }^{\zeta}$ (black dash); (b) resulting absolute value of the second order nonlinearity $\left(\chi^{(2)}\right)$ obtained by power relationship (blue curve) and the comparative method using KTP crystal (red curve) ...............................92

Figure 4.4. (a) Normalized $\chi_{2 D}^{(2)}$ versus SH photon energy (black curve). Green solid curve is the result of $\chi_{2 D}^{(2)}$ calculations for $E_{g}=2.21 \mathrm{eV}$ while changing the parameter to 
$2.15 \mathrm{eV}$ results in the blue dash-dotted curve. (b) Experimental $\chi_{2 D}^{(2)}$ dispersion (black line) and result of calculations accounting for contributions due to exciton states with energies $E_{n}$ below the bandgap. Colored (dashed) lines show contributions to the nonlinearity from broadened ( $\Delta v_{n}=35-42 \mathrm{meV}$ ) exciton lines with the main quantum

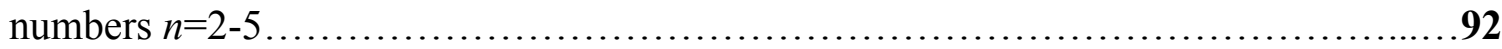

Figure A.1. OPO output pulse characteristics: (a) shows OPO pulse spectra obtained throughout available tuning range for the OPO. (b) (c) Shows typical pulse autocorrelations for signal (b) and idler (c) beams. (d) Shows idler pulse spectrum at $\sim 4.5$ micron while OPO can still deliver $\sim 50 \mathrm{~mW}$ of average power in idler branch..........98 Figure A.2. The characteristic curve of a broadband pulse generated by PCF: (a) Fundamental pulse of TiS at $750 \mathrm{~nm}$. (b) The output broadband pulse when the $750 \mathrm{~nm}$ pulse with energy $150 \mathrm{~mW}$ passes through PCF .................................99

Figure A.3. Actual setup configuration for the supercontinuum generation setup.........99

Figure B.1. Typical cross-sectional geometry of a PANDA fiber. The core is a singlemode waveguide and the inner cladding is multi-mode waveguide. The stress induced birefringence (dark blue) allows for polarization maintaining properties for linearly

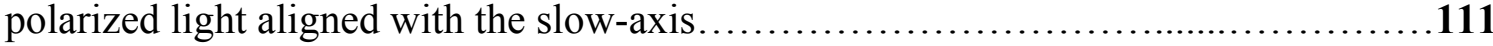
Figure B.2. Schematic diagram of cladding-pumping. (a) Pump light propagates through multi-mode inner cladding waveguide and is absorbed by $\mathrm{Yb}$ ions upon contact increasing population inversion. (b) Light reflection in double-clad fiber at a perpendicular fiber termination and at an angled fiber termination. Incident light is in solid red and reflected light dotted red..........................................111 
Figure B.3. Schematic diagram of a Mach-Zehnder modulator. Seed DBR laser light (red) enters modulator, the waveguide splits into to two paths and recombined onto an output waveguide generating intensity modulation at output. Generated waveguide (orange) driven by arbitrary function generator and DC bias voltage at quadrature point

Figure B.4. Schematic diagram of the grating in the Littrow configuration. Free-running lasing mode at $1072 \mathrm{~nm}$ (red) are incident on the grating and the reflection (dotted red) are scattered and not spectrally selected to propagate back into the active fiber. The seeded mode at $1064 \mathrm{~nm}$ (orange) is reflected back off of the grating and tuned to propagate back

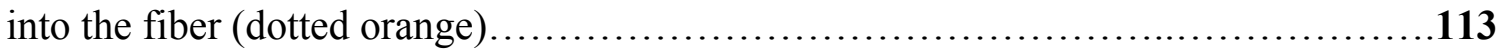

Figure B.5. Schematic of experimental CARS setup. ISO: isolator; WP: wave plate; GT: Glan-Thompson polarizer; BS: beamsplitter; HR: high reflector mirror; M: mirror; CR: crystal; OC: output coupler; DCM: dichroic mirror..............................113 Figure B.6. Image of actual CARS experimental setup.............................114 Figure B.7. Schematic diagram of image/data acquisition system. GR: grating; OBJ: objective; CC: corner cube; CL: collimating lens; BP: bandpass filter...................115 Figure B.8. Schematic diagram of a SHG process. An incident pump pulse (solid red) with optical frequency $\omega$ passes through a nonlinear crystal generating forward- and backward-propagating light (dotted blue) with frequency doubled $2 \omega$ and residual pump

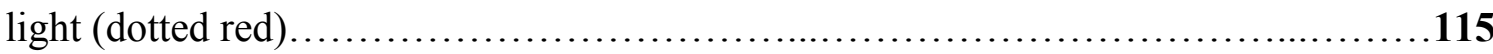




\section{LIST OF ACRONYMS}

\section{CHAPTER 1}

SBS

SBS-QS

FWHM

DCF

SMF

FBG

$\mathrm{AOM}$

$\mathrm{Cr}^{4+}: \mathrm{YAG}$

SA

PANDA

DBR

MZ

$\mathrm{dB}$

Vpi

RF

DC

HR

CW

ASE

ISO
Stimulated Brillouin scattering

Stimulated Brillouin scattering based Q-switched

Full width at half max

Double-clad fiber

Single-mode fiber

Fiber Bragg grating

Acousto-optic modulator

Yttrium aluminum garnet doped with chromium

Saturable absorber

Polarization maintaining and absorption reducing

Distributed Bragg reflector laser

Mach-Zehnder

Decibel

Voltage $\pi$

Radio frequency

Direct current

High reflecting

Continuous wave

Amplified spontaneous emission

Isolator 


$\begin{array}{ll}\text { VA } & \text { Variable attenuator } \\ \text { PM } & \text { Power meter } \\ \text { OSA } & \text { Optical spectrum analyzer } \\ \text { BS } & \text { Beam splitter } \\ \text { LD } & \text { Laser diode } \\ \text { AL } & \text { Aspheric lens } \\ \text { TM } & \text { Transverse magnetic }\end{array}$

\section{CHAPTER 2}

KTP

SRS

CARS

OPO

PPSLT

NA

PMT

FT

NSF

CHAPTER 3

CARS

KTP

PPSLT
Potassium titanyl phosphate

Stimulated Raman scattering

Coherent anti-Stokes Raman spectroscopy

Optical parametric oscillator

Periodically poled lithium tantalite crystal

Numerical aperture

Photomultiplier tube

Fourier transform

National science foundation
Coherent anti-Stokes Raman spectroscopy

Potassium titanyl phosphate

Periodically poled lithium tantalite crystal 


$\begin{array}{ll}\text { OBJ } & \text { Apochromat objective lens } \\ \text { NA } & \text { Numerical aperture } \\ \text { PMT } & \text { Photomultiplier tube } \\ \text { DAQ } & \text { High-speed data acquisition card } \\ \text { FWHM } & \text { Full width at half max } \\ \text { RBC } & \text { Red blood cells } \\ \text { GR } & \text { Diffraction grating } \\ \text { BP } & \text { Bandpass filter } \\ \text { CL } & \text { Collimating lens }\end{array}$

\section{CHAPTER 4}

$\begin{array}{ll}\text { SHG } & \text { Second harmonic generation } \\ \text { SH } & \text { Second harmonic } \\ \text { 2D } & \text { Two dimension } \\ \text { IR } & \text { Infrared } \\ \text { CCD } & \text { Charge coupled device } \\ \text { Ti:sapphire } & \text { Titanium sapphire laser } \\ \text { BZ } & \text { Brillouin zone } \\ \text { NSF } & \text { National science foundation } \\ \text { ISO } & \text { Isolator } \\ \text { PCF } & \text { Photonic crystal fiber } \\ \text { DCM } & \text { Dichroic mirror } \\ \text { OBJ } & \text { Apochromat objective lens }\end{array}$




\section{APPENDIX A}

$\begin{array}{ll}\text { SBS } & \text { Stimulated Brillouin scattering } \\ \text { NA } & \text { Numerical aperture } \\ \text { DBR } & \text { Distributed Bragg reflector laser } \\ \text { InGaAs } & \text { Quantum well laser diode } \\ \text { RIN } & \text { Relative intensity noise } \\ \text { PM } & \text { Polarization maintaining } \\ \text { Ti:sapphire } & \text { Titanium sapphire laser } \\ \text { CARS } & \text { Coherent anti-Stokes Raman spectroscopy } \\ \text { OPO } & \text { Optical parametric oscillator } \\ \text { PPSLT } & \text { Periodically poled lithium tanatalte } \\ \text { CW } & \text { Continuous wave } \\ \text { Ti:S } & \text { Titanium sapphire laser } \\ \text { PPLN } & \text { Periodically poled lithium niobate } \\ \text { SHG } & \text { Second harmonic generation } \\ \text { PCF } & \text { Photonic crystal fiber }\end{array}$

\section{APPENDIX B}

SBS

PANDA

HR
Stimulated Brillouin scattering

Polarization maintaining and absorption reducing High reflecting 


\begin{tabular}{|c|c|}
\hline DBR & Distributed Bragg reflector laser \\
\hline $\mathrm{RF}$ & Radio frequency \\
\hline $\mathrm{DC}$ & Direct current \\
\hline CARS & Coherent anti-Stokes Raman spectroscopy \\
\hline OPO & Optical parametric oscillator \\
\hline Ti:sapphire & Titanium sapphire laser \\
\hline NA & Numerical aperture \\
\hline PMT & Photomultiplier tube \\
\hline DAQ & High-speed data acquisition card \\
\hline $\mathrm{BBO}$ & Beta-barium borate nonlinear optical crystal \\
\hline SFG & Sum frequency generation \\
\hline DCM & Dichroic mirror \\
\hline SHG & Second harmonic generation \\
\hline PCF & Photonic crystal fiber \\
\hline UV & Ultraviolet \\
\hline IR & Infrared \\
\hline SPF & Shortpass filter \\
\hline $\mathrm{CCD}$ & Charge coupled device \\
\hline USB & Universal serial bus \\
\hline MM & Multi-mode \\
\hline SM & Single-mode \\
\hline ISO & Isolator \\
\hline WP & Waveplate \\
\hline
\end{tabular}




$\begin{array}{ll}\text { GT } & \text { Glan-Thompson } \\ \text { BS } & \text { Beamsplitter } \\ \text { HR } & \text { High reflecting } \\ \text { M } & \text { Mirror } \\ \text { CR } & \text { Crystal } \\ \text { OC } & \text { Output coupler } \\ \text { GR } & \text { Grating } \\ \text { OBJ } & \text { Apochromat objective lens } \\ \text { CC } & \text { Corner cube } \\ \text { CL } & \text { Collimating lens } \\ \text { BP } & \text { Bandpass filter }\end{array}$




\section{CHAPTER 1}

Submitted to OSA Continuum

Seeded Yb-doped Q-switched fiber laser based on stimulated Brillouin scattering (SBS)

Adam Card and Feruz Ganikhanov

Department of Physics, University of Rhode Island, 2 Lippitt Road, Kingston, RI, 02881.

Corresponding Author: $\quad$ Feruz Ganikhanov, Ph. D.

Department of Physics

University of Rhode Island

East Hall, 2 Lippitt Road

Kingston, RI, 02881, USA

Phone: (401) 874-2633

Email address: fganikhanov@uri.edu 
Q-switched fiber lasers which make use of stimulated Brillouin scattering (SBS) as a passive Q-switching mechanism have been the focus of a significant amount of research efforts and development since their inception in 1997 [1]. In fact, as of the writing of Ref. 2 in 2017, the amount of research papers published under the topic ‘Stimulated Brillouin' was a total of 4,243 papers [2]. SBS based Q-switched (SBS-QS) fiber lasers have displayed a novel ability to compress Q-switched pulses to less than a few ns pulse width (FWHM) as well as high-peak-power, often in excess of $10 \mathrm{~kW}$, which are several orders of magnitude narrower and greater, respectively, than pulses generated by conventional Q-switched fiber lasers [1, 3-8]. The addition of Yb-doped double-clad fiber (DCF) to SBS-QS fiber laser models has been pivotal in the advancement of high-power fiber lasers. Cladding pumping due to Yb-doped DCF technology allows for high-power broad stripe, relatively cheap laser diodes to be coupled into active fiber with superior launching efficiency. High-intensity light confined within the single-mode, Yb-doped core due to cladding pumping allows for SBS-QS fiber lasers to easily reach power thresholds of various nonlinear effects, such as low-threshold SBS. The combination of cladding pumping and Q-switching allows for high-energy, high-peak-power, short pulse emission in regular or irregular self-pulsing regimes [7-11]. These types of fiber lasers have proved to be very beneficial in the field of medicine, industrial processing, range finding, and remote sensing, as well as in applications such as LIDAR [4].

Of course, utilizing SBS as a Q-switching mechanism comes with a few considerable drawbacks. In single-mode fiber (SMF), SBS is due to stochastic thermal noise fluctuations within the medium density of the fiber [12] with the driving optical 
field being random superposition of laser modes in the cavity. The noise source constituting these thermal fluctuations in the medium density, known as the Langevin noise source, is assumed to be a Gaussian random variable with zero mean and is $\delta$ correlated in space and time. In short, output laser characteristics in SBS-QS fiber lasers, unless externally controlled, are strongly affected by an initial distribution of laser modes in the cavity and by thermal noise. Because of this characteristic, the output pulse train of SBS-QS fiber lasers has considerable repetition rate instability and corresponding pulse-to-pulse intensity fluctuations. It has been shown experimentally that the spacing between adjacent Stokes pulses can vary greatly. In some cases, the variance of the repetition rate in SBS-QS fiber lasers was found to be $\sim 20 \%$ of the period [14]. Additionally, the intensity of the Stokes output has been shown to exhibit 100\% temporal fluctuations in the sense that the normalized standard deviation of the pulse intensity is equal to unity [13].

A considerable amount of experimental and theoretical efforts have been undertaken in order to enhance SBS-QS fiber laser output as well as to overcome its glaring deficiencies. Enhancements of the actual fiber cavity design itself have been numerous. Fiber Bragg gratings (FBG) spliced onto the back end of active fiber cavities have been used in order to introduce narrow spectrum feedback in an effort to more efficiently generate SBS (in the case of an Yb-doped DCF core, the back-reflected light being approximately $1.06 \mu \mathrm{m}$ wavelength) $[8,11,16,18]$. Often in experimental setups, several tens of meters of passive fiber are spliced to the active fiber in order to enhance the distributed backscattering SBS effect or, in some cases, the passive fiber itself served as a back mirror utilizing narrow-spectrum Rayleigh scattered light to retro-reflect back 
into the active fiber $[1,4,6,16-17]$. There have also been studies using a 10/90 coupler in the form of a Rayleigh-stimulated Brillouin scattering ring interferometer. The ring interferometer acts as a spectrally-selective resonating energy source which enhances a cavity mode until it reaches SBS threshold, successfully generate nanosecond pulses, much shorter than the round-trip time of the fiber resonator length [14, 18]. External Qswitching mechanisms, typically in the form of acousto-optic modulators (AOM) or $\mathrm{Cr}^{4+}$ YAG saturable absorbers (SA), have been added to the all-fiber cavity design as well in order to enhance SBS giant pulse peak-power and to stabilize the repetition rate by forcing SBS initiation by increasing HR feedback once the fiber is inverted to a high level [3-6, 15, 19]. In addition to adjustments in laser cavity design, SBS-QS repetition rate has been stabilized experimentally using a pulsed pump laser diodes $[7,20]$ and, in the case most relevant to our current work, by externally injecting square-wave modulated light into the fiber cavity [14]. The latter study showed great success in developing a novel method of gain control in SBS-QS fiber lasers, drastically improving repetition rate variance from about $20 \%$ of the period to about $1 \%$ of the period [14].

For sufficiently intense incident light, with a spectral width within a few tens of $\mathrm{MHz}$, a nonlinear SBS mirror can be efficiently generated in single-mode fiber. The SBS mirror produces fast transient backscattered Stokes feedback and can convert more than $80 \%$ of incident intensity into the narrow Stokes pulse intensity [23].

Our current study aims to improve upon previous experimental stabilization efforts by injecting periodic waveforms into the fiber cavity that will govern the nonlinear SBS mirror and the Q-switching effect. The externally seeded optical mode with optimized parameters (i.e. amplitude and bandwidth) is expected to significantly 
suppress the stochastic nature of laser generation and initiation of the SBS effect that is behind the Q-switching mechanism in this type of laser. In other words, the optimized optical mode seeded is expected to control initial laser dynamics that yields in a stable output pulsed train synchronized to the frequency of the seeded waveform in each cycle of Q-switched pulse generation. Figure 1.1 (a) shows the theoretical interpretation of our current experimental investigation. The $1064 \mathrm{~nm}$ waveform with frequency $\omega_{\mathrm{L}}$ (orange), is injected into the core of highly inverted Yb-doped DCF. The waveform is amplified as it traverses the inverted fiber until its intensity reaches the SBS threshold. At this point, the nonlinear SBS mirror is generated where the acoustic sound wave (grey) has driven frequency $\Omega$ and the backscattered Stokes wave (red) has a downshifted frequency $\left(\omega_{L^{-}}\right.$ $\Omega$ ). If the active fiber is pumped above transparency level (for small signal gain) and there is sufficient cavity feedback, a waveform single passing through the gain fiber with sufficient length can at the same time generate a Stokes mode/pulse that can in turn generate an efficient nonlinear SBS mirror. This can further create a subsequent Stokes ordered pulse propagating in the opposite direction. This process, known as the cascaded SBS effect, is depicted in Figure 1.1 (b) and typically generates Stokes orders until the population inversion is essentially depleted.

The experimental setup is shown in Figure 1.2. Our experiment has two primary aspects, the SBS laser configuration and the injection setup. The SBS laser is pumped by a $976 \mathrm{~nm}$ pump laser diode (EM4) with a pump power up to $5 \mathrm{~W}$ (blue). The pump light passes through a dichroic mirror and is coupled into the active $\mathrm{Yb}$-fiber. The active fiber (Thorlabs) is $\sim 6.2 \mathrm{~m} \mathrm{Yb-doped} \mathrm{DCF} \mathrm{with} \mathrm{a} \mathrm{core} \mathrm{diameter} \mathrm{of} 6 \mu \mathrm{m}$ and an absorption coefficient of $\sim 2.4 \mathrm{~dB} / \mathrm{m}$ near $976 \mathrm{~nm}$ wavelength. The inner cladding has a diameter of 
$125 \mu \mathrm{m}$ and is in the PANDA polarization-maintaining geometry. Both ends of the fiber had perpendicular termination which made a 4\% Fresnel reflection feedback at either end of the fiber. This type of fiber termination is not ideal, however we did not have the equipment necessary to perform angle cleaves on double-clad fiber. The front end of the PANDA fiber was rotated to align the slow-axis of the fiber cross section with the vertical polarization of the injected light (Figure 1.2 inset). This maximized the effectiveness of the injection. The back end of the cavity was composed of a diffraction grating in the Littrow configuration whereby the $1064 \mathrm{~nm}$ injected mode was spectrallyselected to reflect back into the active fiber while other free-running SBS lasing modes were scattered and did not propagate back into the fiber. A variable attenuator was added to the fiber cavity to suppress feedback and enhance the SBS generation.

The injection setup begins with a 1064nm seed DBR laser diode (EM4) with an output power of $150 \mathrm{~mW}$ and spectral width $10 \mathrm{MHz}$ that is fiber coupled into the MachZehnder (MZ) modulator (EOSpace). The MZ modulator has a $3.0 \mathrm{~dB}$ insertion loss, bias Vpi of $1.9 \mathrm{~V}$ and an extinction ratio of $25 \mathrm{~dB}$. The RF signal is driven by an arbitrary function generator (Tektronix) and there is a DC Bias voltage set at the quadrature (half-max) point. This allows for many different types of waveforms to be generated with variable frequency and period. Light leaves the MZ modulator, exits a fiber collimator and passes through a two-lens telescopic system to narrow the beam waist. The light then exists through a free-space isolator (OFR) with an $\sim 2.7 \mathrm{~mm}$ max beam diameter with up to $44 \mathrm{~dB}$ isolation. The injection light (orange) passes through a $50 / 50$ beamsplitter and half of the light goes to measurement and the other half is coupled into the active fiber. Non-absorbed injection light is then reflected back into the fiber 
cavity by the diffraction grating in Littrow configuration. Measurement of the injection light is composed of a power meter (Newport), an optical spectrum analyzer (Anritsu, $0.05 \mathrm{~nm}$ maximum resolution), a photodetector (Thorlabs, $150 \mathrm{MHz}$ bandwidth) coupled into an oscilloscope (Tektronix, $350 \mathrm{MHz}$ bandwidth). The SBS laser output (red) passes through the beamsplitter and is measured by the same power meter and optical spectrum analyzer as mentioned previously, as well as a fast photodetector (Thorlabs, $5 \mathrm{GHz}$ bandwidth) fiber coupled into the oscilloscope. This allows for both the injected waveform and the SBS lasing pulse train to be viewed on the oscilloscope at the same time. The resolution of the fast photodetector/oscilloscope combination allows for a maximum $1 \mathrm{~ns}$ rise time measurement of individual SBS pulses.

We began by experimenting with the type of back mirror in the fiber laser configuration in order to get a free-running stochastic SBS lasing effect which very clearly showed characteristic repetition rate and intensity fluctuations in the fiber laser. Initially, we choose to use an HR mirror as the back mirror of the cavity (Figure 1.3). With a low pump power of $433 \mathrm{~mW}$, the oscilloscope trace in Figure 1.3(a) shows evidence of self-pulsing relaxation oscillations. As the pump power increases in pump power shown in Figure 1.3 (b)-(d), the oscilloscope trace shows that the HR feedback is far too large to allow for the transient dynamics of SBS and we see an output of mostly $\mathrm{CW}$ lasing and no evidence of SBS. Output power for the HR mirror configuration is shown in to have a somewhat linear relationship with the pump power (see Figure 1.4 (a)). The spectrum output for low pump (Figure 1.4(b)) shows a few spectral components with maximum gain at $1075 \mathrm{~nm}$ wavelength. As the pump power increases (Figure 1.4 
(c)), the output spectrum shows a broad spectral cluster for $\mathrm{CW}$ lasing from approximately $1073 \mathrm{~nm}$ to $1081 \mathrm{~nm}$ with a largest gain at approximately $1075 \mathrm{~nm}$.

We then decided to block the HR mirror in the cavity completely, allowing the two perpendicularly cleaved fiber terminations with corresponding 4\% Fresnel reflection as the only source of cavity feedback. The oscilloscope traces with this bare-fiber cavity configuration with varying pump power is shown in Figure 1.5. For relatively low pump power $762 \mathrm{~mW}$ (Figure 1.5 (a)), we see some self-pulsing relaxation oscillations and as the pump power increases to $1.08 \mathrm{~W}$ (Figure $1.5(\mathrm{~b})$ ), the relaxation oscillations increase in amplitude and repetition rate. As the pump power exceeds $\sim 1.3 \mathrm{~W}$, seen in Figure 1.5 (c), the relaxation oscillation pulses have collapsed into SBS pulses. We call this pump power level the SBS threshold. We see in this image the narrow, high-peak-power Stokes pulses with the instability in repetition rate and pulse-to-pulse intensity. As the pump power far exceeds the SBS threshold (Figure 1.5 (d)), the repetition rate of the SBS pulses increases by a factor of 2 .

Figure 1.6 shows the output of the free-running SBS laser with the bare-fiber cavity configuration. In Figure 1.6 (a), we see the output power as a function of the pump power. Before the pump power reaches SBS threshold $(\sim 1.3 \mathrm{~W})$, we see the pre-SBS lasing linear relationship and when the pump power exceeds the SBS threshold, the slope of the linear relationship corresponding to SBS increases significantly. Figure 1.6 (b) shows the output spectrum for pump power $954 \mathrm{~mW}$, less than threshold. In this image there are a few spectral components where the largest gain corresponds to approximately $1072 \mathrm{~nm}$. As the pump power increases past threshold in Figure 1.6 (c), the output spectrum shows several different spectral components with relatively high gain values 
but the maximum gain value is still approximately corresponding to $1072 \mathrm{~nm}$. This image shows that SBS pulsing exhibits unstable wavelength lasing patterns that our injection experiment will ultimately stabilize.

Because the highest gain during free-running SBS lasing in the bare-fiber cavity configuration is at approximately $1072 \mathrm{~nm}$, we encountered an experimental difficulty. The central wavelength of our injected light was approximately $1064.6 \mathrm{~nm}$, as shown in Figure 1.7 (a), which did not line up with the wavelength of the largest gain for freerunning SBS. That being the ideal condition for the effectiveness of the injection. We did not have the equipment required to tune the injection wavelength to match that of the highest free-running gain. The broad spectrum of ytterbium is shown in the ASE spectrum for pump power slightly above lasing threshold in Figure 1.7 (b). In this image, the dotted blue vertical line represents $1072 \mathrm{~nm}$ wavelength of the highest gain and the dotted red vertical line represents the center wavelength of the injected light. This problem was resolved because ytterbium is homogenously broadened and therefore, if we inject with sufficiently high intensity, other free-running SBS modes will be completely suppressed. To test this idea, we injected $\mathrm{CW}$ light with varying loss and detected the output spectrum using a partial mirror as the cavity back mirror. In Figure 1.8 (a), the CW injection had a very high loss and the injected $1064 \mathrm{~nm}$ CW light is slightly more intense than the free-running SBS lasing modes. As the CW injection loss decreases (as shown in Figure 1.8 (b) and (c)), the CW injection spectrum almost completely suppresses the free-running modes. This is an indication that even though we cannot line up the wavelength of the injection and the wavelength of the largest gain, if we inject with sufficient intensity we can still drown out the other free-running SBS modes. 
In order to maximize the effect of the injection, we installed a diffraction grating that will provide wavelength dependent feedback (analogous to a fiber Bragg grating). The grating is 1200 lines $/ \mathrm{mm}$ and is used in the Littrow configuration at an angle that favors the diffracted mode being at a wavelength that matches as precisely as possible the wavelengths of the seeded optical mode. Figure 1.9 shows the oscilloscope traces for the Littrow configuration with varying pump power. Figure 1.9 (a) shows self-pulsing relaxation oscillations with a low pump power of $824 \mathrm{~mW}$. As the pump power was increased from $824 \mathrm{~mW}$ to $1.08 \mathrm{~W}$ (Figure 1.9 (b)), the relaxation oscillation mode grows with no indication of its development into short-pulse mode due to SBS Q-switching. The laser dynamics are entirely affected by $\mathrm{CW}$ mode generation that depletes the optical gain. As the pump power is increased further to $1.47 \mathrm{~W}$ then to $1.84 \mathrm{~W}$, shown in Figure 1.9 (c) and (d), the relaxation oscillation self-pulsing is further amplified and the repetition rate increases further and some SBS pulses are seen. The transient dynamics of SBS are not seen clearly for this configuration so a variable attenuator was added to the fiber cavity to introduce cavity losses and enhance the SBS lasing effect. Figure 1.10 (a) shows two distinct linear relationships for the output power as a function of pump power. The output spectrum, shown in Figure 1.10 (b), shows a tuned mode at approximately $1066 \mathrm{~nm}$ which completely suppresses all other free-running modes $(1072 \mathrm{~nm}-1074 \mathrm{~nm})$. Figure 1.10 (c) shows the tuned output spectrum between $1066 \mathrm{~nm}$ to $1066.5 \mathrm{~nm}$.

Figure 1.11 shows the oscilloscope traces for free-running SBS lasing effect with Littrow configuration and cavity losses added. For low pump power $824 \mathrm{~mW}$ in part (a), we see relaxation oscillation self-pulsing and as the pump power increases to $1.08 \mathrm{~W}$ in part (b), those relaxation oscillation pulses clearly increases in amplitude and repetition 
rate. As the pump power increases past SBS threshold $(\sim 1.3 \mathrm{~W})$, as shown in Figure 1.11 (c), the SBS mode is favored (i.e. experiencing more gain than CW) and several different Stokes pulses are evident. As pump power increases further to $1.84 \mathrm{~W}$, as shown in Figure 1.11 (d), we see an oscilloscope trace composed entirely of Stokes pulses with varying pulse-to-pulse intensity and unstable repetition rate. This cavity configuration was deemed suitable for our experimental purposes.

The output measurements for the free-running SBS with the Littrow configuration with cavity losses are shown in Figure 1.12. In Figure 1.12 (a), the output power as a function of pump power shows two clear linear regression lines. For pump power values less than SBS threshold $(\sim 1.3 \mathrm{~W})$, we see a linear relationship for pre-SBS lasing and as the pump power exceeds threshold, we see a significantly larger slope corresponding to SBS lasing. In Figure 1.12 (b) and (c), the output spectrum is shown with a broad span and a narrow span, respectively, for a pump power greater than threshold. We see in these spectra the tuned wavelength reinforced by the diffraction grating in the Littrow configuration at $\sim 1066 \mathrm{~nm}$ which is significantly less intense than the free-running SBS modes as a result of the added cavity losses.

Figure 1.13 shows the single SBS pulses in the free-running SBS regime prior to injection. In Figure 1.13 (a) and (b), we see a single SBS pulse in the bare-fiber termination configuration where a single SBS pulse is visible with two smaller sub-pulses due to the Fresnel reflection off of the perpendicular termination. Indeed, with a fiber of length $\sim 6.2 \mathrm{~m}$ the round trip time is approximately $61 \mathrm{~ns}$. Part (b) shows the SBS pulse width (FWHM) to be less than 2 ns (keeping in mind the 1 ns rise time limitation of our oscilloscope setup). Figure 1.13 (c) and (d) show the single SBS pulse for the diffraction 
grating and cavity losses configuration. Part (c) shows a cascaded SBS effect with 5 or 6 Stokes pulses evident and part (d) shows that the Stokes pulse has $<4$ ns pulse width (FWHM). This is a curious result, as we were expecting a pulse width $<2 \mathrm{~ns}$. We believe this is because of the fluctuating pulse measurements due to the stochastic SBS pulse generation.

The first type of waveform injection experimented with was a continuous sine wave modulated light with a frequency of $160 \mathrm{kHz}$. Figure 1.14 (a) and (b) shows the oscilloscope traces with the sine wave injection (blue) and the Stokes pulses (yellow). The output pulse train shows the SBS repetition rate is largely synchronized to the frequency of the sine wave modulated light injection. Figure 1.14 (c) and (d) depicts a single SBS pulse with the sine wave injection. Figure 1.14 (c) shows a cascaded SBS effect with 3 or 4 Stokes orders, however it is far less pronounced than the cascaded SBS effect for the free-running SBS Littrow configuration with loss shown in Figure 1.14 (c). Figure 1.14 (d) shows an SBS pulse width (FWHM) of $<2$ ns.

The output measurements for the sine wave modulated light injection are shown in Figure 1.15. The output power as a function of pump power shown in Figure 1.15 (a) identifies two distinct relationships. For pump power less than threshold $(\sim 1.1 \mathrm{~W})$, there is a quadratic relationship and then after threshold is achieved, the characteristic linear relationship is observed for SBS lasing. This implies that the sine wave undergoes amplification due to double-passing through the inverted fiber until it has an intensity large enough to seed the SBS mechanism itself. For all pump powers in excess of this value, the sine wave seeds the SBS process excluding thermal noise initiation. Figure 1.15 (b) and (c) shows the output spectrum. Figure 1.15 (b) shows that the $\sim 1064 \mathrm{~nm}$ 
spectrum does not completely suppress the modes due to termination at $\sim 1074 \mathrm{~nm}$ as we would have hoped. We did not have amplifier equipment to properly amplify the injection enough to completely suppress the approximately $1074 \mathrm{~nm}$ modes. Figure 1.15 (c) shows a very curious result. The output spectrum (blue line) has a slight blue-shifted wavelength compared to the injection spectrum (dotted red). This implies the output frequency has increased seemingly indicating anti-Stokes pulse generation instead of Stokes pulse generation. The top of the output pulse in Figure 1.15 (c) also shows evidence of several Stokes orders but the spectrum analyzer has a max resolution of 0.05 $\mathrm{nm}$ and we cannot detect the exact shape. We are currently looking into this unusual effect.

The second type of waveform we experimented with was a continuous triangular wave modulated light with a frequency of $130 \mathrm{kHz}$. Figure 1.16 (a) and (b) show the oscilloscope traces of the typical pulse train corresponding to the triangular wave modulated light injection. The SBS pulse (yellow) repetition rate is completely synchronized to the frequency of the injection (blue). Figure 1.16 (c) and (d) show a single SBS pulse for the triangular wave injection. The single SBS pulse shows a cascaded SBS effect with a few Stokes orders but is not as pronounced as the freerunning SBS grating and losses configuration shown in Figure 1.11 (c). Figure 1.16 (d) shows the pulse width (FWHM) in the presence of the triangular wave injection to be $<2$ ns.

The output measurements for the continuous triangular wave modulated light injection are shown in Figure 1.17. Figure 1.17 (a) shows the output power as a function of the pump power. We see two very distinct relationships here. For pump power less 
than threshold $(\sim 1.2 \mathrm{~W})$, we see a quadratic relationship and for pump power greater than the threshold, see a linear relationship that is characteristic of SBS lasing. We believe this is due to the triangular wave becoming amplified as it double-passes the inverted fiber until it is sufficiently amplified to seed the SBS mechanism. We also think that the bandwidth of the injected mode is better matched to seed the power into intrinsic cavity modes that are further amplified and serve as an efficient driving field for the SBS effect. Figure 1.17 (b) shows the injection intensity is nearly large enough to completely subdue the lasing modes at $\sim 1074 \mathrm{~nm}$ and is more effective at suppressing these modes than the sine wave injected light. If we had a pulse amplifier we could completely suppress these extraneous modes but this was not available to us. Figure 1.17 (c) gives a very similar curious result as we have seen in the sine wave injection output. The output pulse (blue) has slightly blue-shifted wavelength relative to the injected spectra (dotted red). We would have expected a larger wavelength, as is indicative of a Stokes order, and this result seems to indicate that the output is due to an anti-Stokes reaction. We also see several Stokes orders in the peak of the output pulse but we can only make out $0.05 \mathrm{~nm}$ maximum resolution from our spectrum analyzer. We are currently looking into this result. A high spectra resolution Fabry-perot etalon is needed to study this effect. The last type of waveform that showed success was an inverted half-sine wave with periodic CW lasing between half-sine waves. The half-sine width (frequency) was $160 \mathrm{kHz}$ and $\mathrm{CW}$ lasing with a $15 \mu$ seriod. This dynamic waveform was made so that the stretch of CW lasing was long enough to deplete the population inversion between SBS pulses by cross gain modulation and to suppress spontaneous Brillouin scattering. The half-sine wave was then made narrow enough to be the exact amount of time it takes 
for SBS to be generated. This injection was used with the bare-fiber termination cavity configuration prior to the addition of the diffraction grating. By making these adjustments to this dynamic waveform, we were able to synchronize the SBS repetition rate to the waveform frequency, as seen in Figure 1.18 (a) and (b). Figure 1.18 (c) and (d) show the individual SBS pulse with this injection. We see in Figure 1.18 (c) that there is one Stokes pulse and two sub-pulses due to Fresnel reflection at fiber termination. The Stokes pulse is $<2$ ns pulse width (FWHM) as shown in Figure 1.18 (d).

Figure 1.19 shows the output measurements for the half-sine modulated light injection. Figure 1.19 (a) shows the output power as a function of the pump power. It is obvious that the power-power data have at least two distinct slopes that can be explained as a strong interference between SBS lasing at seeded $(1064.6 \mathrm{~nm})$ and lasing wavelength corresponding to higher gain $(\sim 1072 \mathrm{~nm})$. The SBS threshold is larger than the threshold values corresponding to the previous two waveforms due to the addition of the grating. Figure 1.19 (b) shows the output spectrum ( 1064 nm) nearly suppressing the termination modes at $\sim 1074 \mathrm{~nm}$. Figure 1.19 (c) shows the blue-shifted output pulse (blue), with what appears to be Stokes orders on its peak that cannot be made out due to the maximum resolution of the spectrum analyzer, compared to the injection spectrum (dotted red). This result was unexpected and we are currently looking into it further but is important to note that this effect is not due to the grating tuning as this configuration did not include the grating.

In conclusion, we have displayed a novel method of stabilizing the repetition rate instability by seeding the SBS pulses with a periodic waveform. We chose a cavity configuration with a diffraction grating in the Littrow configuration with cavity losses in 
order to maximize the effectiveness of the injection and simultaneously enhancing the generation of the SBS pulsing effect. Injections of continuous sine wave modulated light, continuous triangular wave modulated light and half-sine wave pulse modulated light injections successfully synchronized the SBS repetition rate to the waveform frequency and seeded the SBS mechanism for pump power greater than the threshold values. This project is ongoing and requires more funding and equipment in order to investigate further.

\section{References:}

1. S. V. Chernikov, Y. Zhu, and J.R. Taylor, "Supercontinuum self-Q-switched ytterbium fiber laser,” Opt. Lett. 22, 298-300 (1997).

2. E. Garmire, "Perspectives on stimulated Brillouin scattering," New Journal of Physics 19, 011003 (2017).

3. Y.O. Barmenkov, A.V. Kir'yanov, and M.V. Andres, "Nonlinear dynamics of Ytterbium-doped fiber laser Q-switched using acousto-optical modulator," The Eur. Phys. J. Special Topics 223, 2775-2788 (2014).

4. M. Laroche, H. Gilles, S. Girard, N. Passilly, and K. Ait-Ameur, "IEEE Photonics Technol. Lett. 18, 764-766 (2006).

5. L. Pan, I. Utkin, R. Lan, Y. Godwal, and R. Fedosejevs, "High-peak-power subnanosecond passively Q-switched ytterbium-doped fiber laser,” Opt. Lett. 35, 895-897 (2010). 
6. Z.J. Chen, A.B. Grudinin, J. Porta and J.D. Minelly, "Enhanced Q switching in double-clad fiber lasers," Opt. Lett. 23, 454-456 (1998).

7. Y. Fan, F. Lu, S. Hu, K. Lu, H. Wang, X. DOng, and G. Zhang, “105-kW PeakPower Double-Clad Fiber Laser,” IEEE Photonics Technol. Lett. 15, 652-654 (2003).

8. M. Salhi, A. Hideur, T. Chartier, M. Brunel, G. Martel, and C. Ozkul, "Evidence of Brillouin scattering in an ytterbium-doped double-clad fiber laser," Opt. Lett. 27, 1294-1296 (2002).

9. A. Hideur, T. Chartier, M. Brunel, M. Salhi, C. Ozkul, and F. Sanchez, "Modelock, Q-switch and CW operation of an Yb-doped double-clad fiber ring laser," Opti. Comm. 198, 141-146 (2001).

10. Y. Fan, F. Lu, S. Hu, K. Lu, H. Wang, and G. Zhang, "Narrow-linewidth widely tunable hybrid Q-switched double-clad fiber laser,” Opti. Lett. 28, 537-539 (2003).

11. B. Ortac, A. Hideur, T. Chartier, M. Brunel, G. Martel, M. Salhi, F. Sanchez, "Influence of cavity losses on stimulated Brillouin scattering in a self-pulsing side-pumped ytterbium-doped double-clad fiber laser," Opti. Comm. 215, 389395 (2003).

12. R. W. Boyd, and K. Rzazewski, "Noise initiation of stimulated Brillouin scattering," Phys. Rev. A 42, 5514-5521 (1990).

13. A. L. Gaeta, and R. W. Boyd, "Stochastic dynamics of stimulated Brillouin scattering in an optical fiber," Phys. Rev. A 44, 3205-3209 (1991). 
14. Z. Pan, L. Meng, Q. Ye, H. Cai, Z. Fang, and R. Qu, "Repetition rate stabilization of the SBS Q-switched fiber laser by external injection,” Opti. Expr. 17, 31243129 (2009).

15. L. Pan, I. Utkin, and R. Fedosejevs, "Experiment and Numerical Modeling of High-Power Passively Q-Switched Ytterbium-Doped Double-Clad Fiber Lasers," IEEE Jour. of Quan. Elect. 46, 68-75 (2010).

16. B. N. Upadhyaya, A. Kuruvilla, U. Chakravarty, M. R. Shenoy, K. Thyagarajan, and S. M. Oak, "Effect of laser linewidth and fiber length on self-pulsing dynamics and output stabilization of single-mode $\mathrm{Yb}$-doped double-clad fiber laser," Appl. Opti. 49, 2316-2325 (2010).

17. Y. Tang, X. Li, and Q. J. Wang, "High-power passively Q-switched thulium fiber laser with distributed stimulated Brillouin scattering," Opti. Lett. 38, 5474-5477 (2013).

18. A. A. Fotiadi, P. Megret, and Michel Blondel, "Dynamics of a self-Q-switched fiber laser with a Rayleigh-stimulated Brillouin scattering ring mirror,” Opti. Lett. 29, 1078-1080 (2004).

19. Y. Wang, A. Martinez-Rios, and Hong Po, "Experimental study of stimulated Brillouin and Raman scatterings in a Q-switched cladding-pumped fiber laser," Opti. Fiber Tech. 10, 201-214 (2004).

20. G. L. Keaton, M. J. Leonardo, M. W. Byer, and D. J. Richard, "Stimulated Brillouin scattering of pulses in optical fibers," Opti. Expr. 13351-13365 (2014).

21. Andrey Kobyakov, Michael Sauer, and Dipak Chowdhury, "Stimulated Brillouin scattering in optical fibers," Advances in Optics and Photonics 2, 1-59 (2010). 
22. C. Ye, P. Yan, L. Huang, Q. Liu, and M. Gong, "Stimulated Brillouin scattering phenomena in a nanosecond linearly polarized $\mathrm{Yb}$-doped double-clad fiber amplifier," Laser Phys. Lett 4, 376-381 (2007).

23. Boyd, Robert W. Nonlinear Optics. San Diego: Academic Press, 2003. Print. 


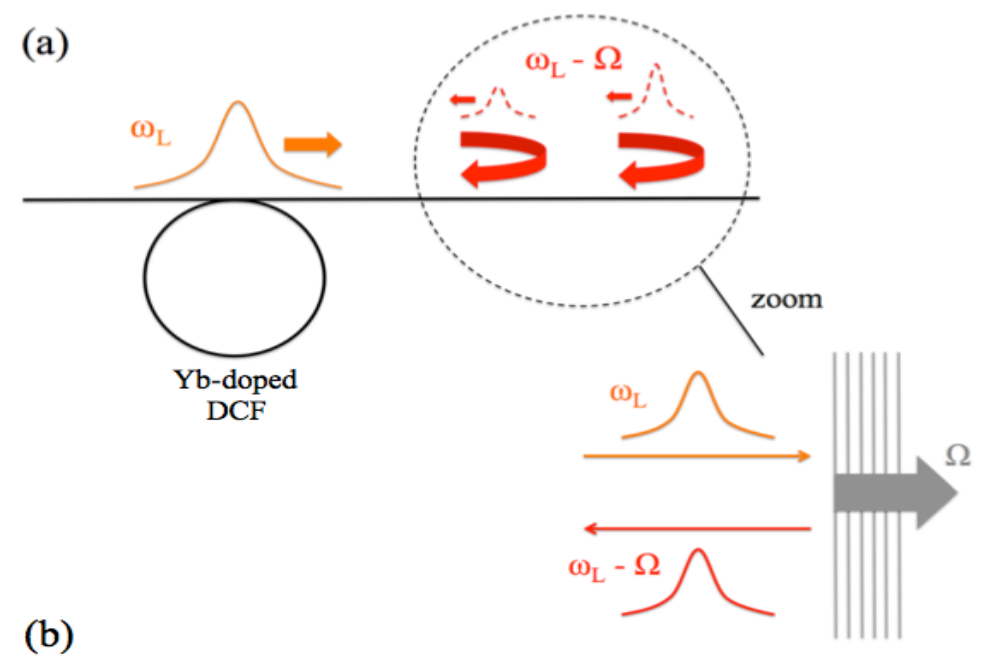

(b)

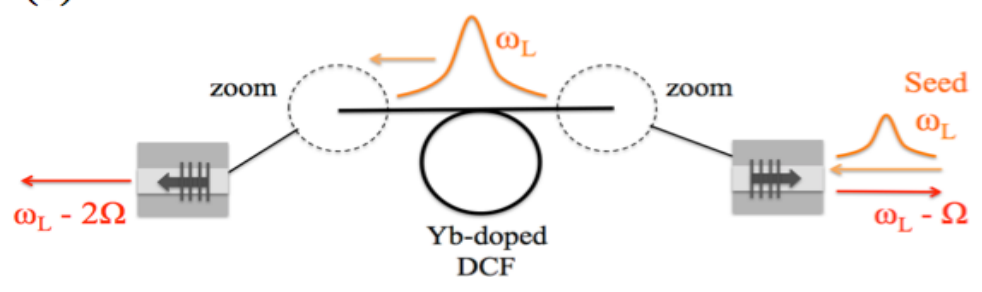

Figure 1.1: Theoretical interpretation of study: (a) $1064 \mathrm{~nm}$ waveform (orange) is injected into inverted fiber and is amplified to SBS threshold as it passes through the fiber generating the nonlinear SBS mirror. Acoustic sound wave is in grey and Stokes light is in red; (b) The cascaded SBS effect is shown where multiple Stokes orders exist creating acoustic sound waves (grey) at either end of the double-clad fiber. 


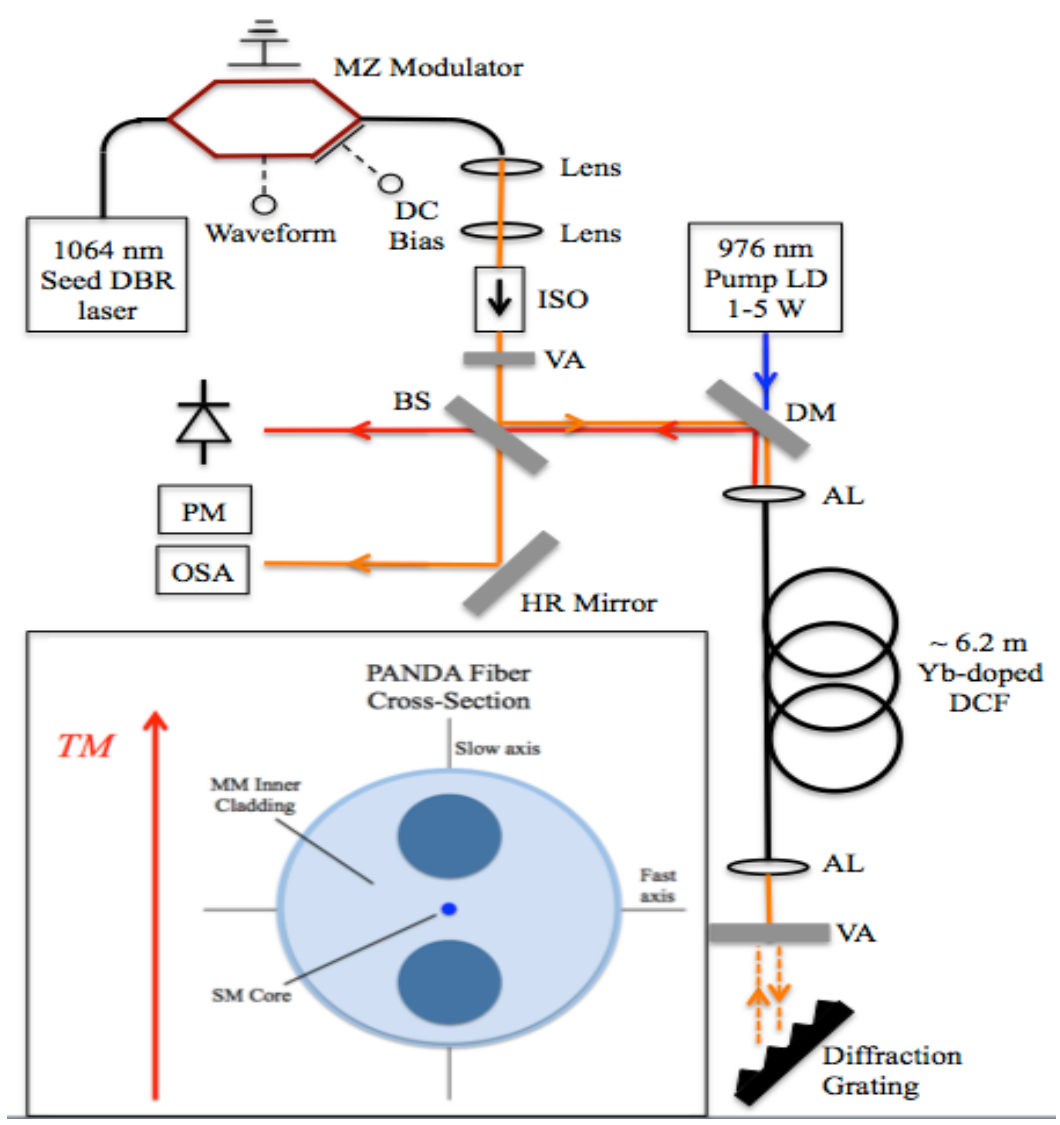

Figure 1.2: Experimental setup. MZ: Mach-Zehnder; ISO: isolator; VA: variable attenuator; PM: power meter; OSA: optical spectrum analyzer; BS: 50/50 beamsplitter; LD: laser diode; DM: dichroic mirror; AL: aspheric lens; TM: transverse magnetic. 

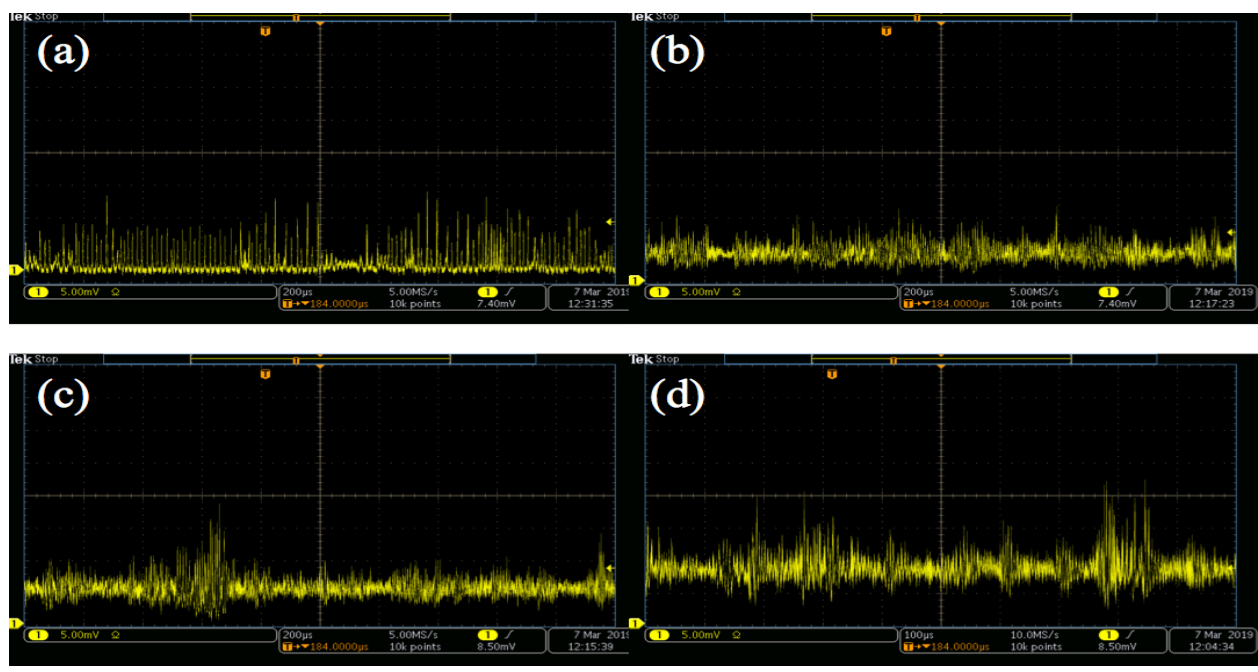

Figure 1.3: Oscilloscope traces of free-running SBS with HR mirror configuration with varying pumpo power. (a) Pump power $433 \mathrm{~mW}$ (b) Pump power 1.08W (c) Pump power $1.4 \mathrm{~W}$ and (d) Pump power 1.84 W.
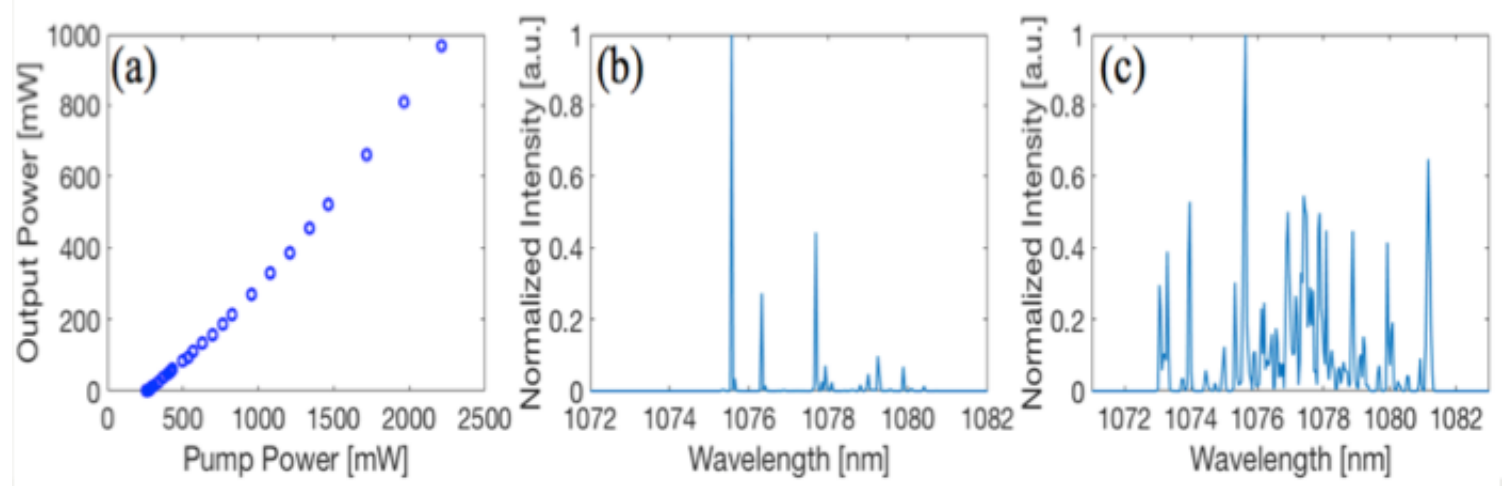

Figure 1.4: Output measurements for the HR mirror cavity configuration: (a) Output power as a function of pump power (b) Output spectrum for low pump power 433mW (c) Output spectrum for high pump power $1.84 \mathrm{~W}$. 

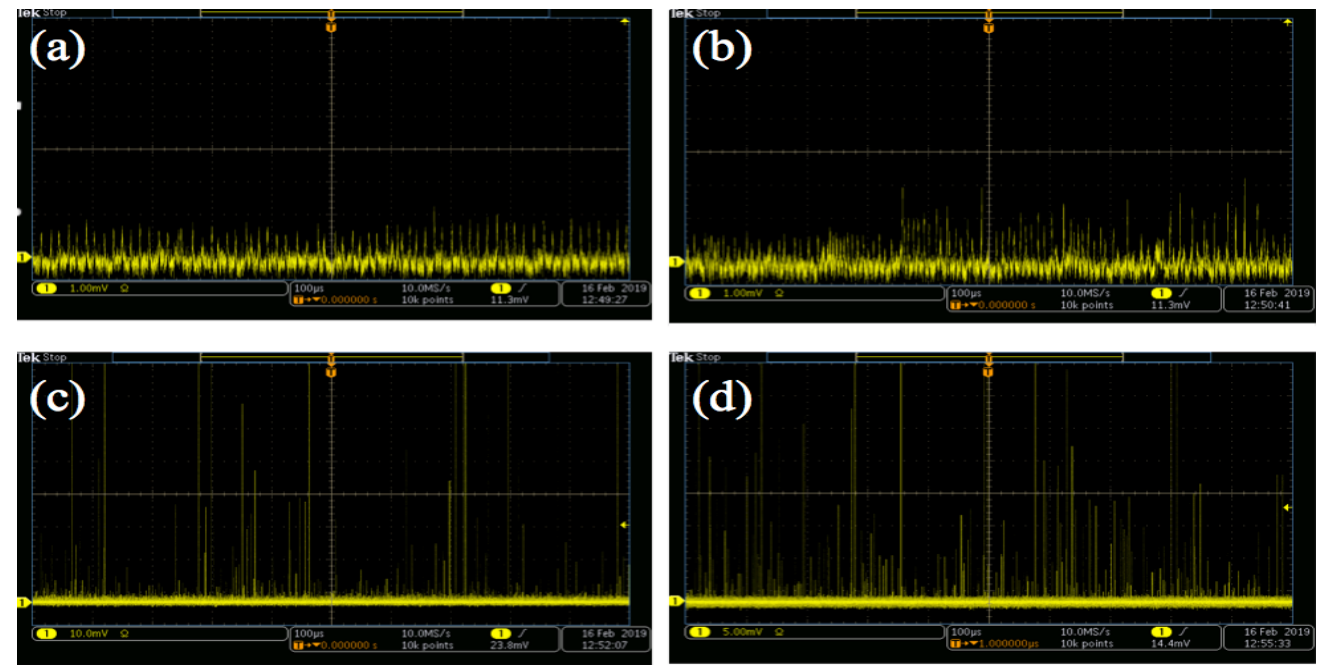

Figure 1.5: Oscilloscope traces of free-running SBS with bare-fiber cavity configuration with varying pump power. (a) Pump power $762 \mathrm{~mW}$ (b) Pump power $1.08 \mathrm{~W}$ (c) Pump power 1.4 W (d) Pump power 2.03 W.
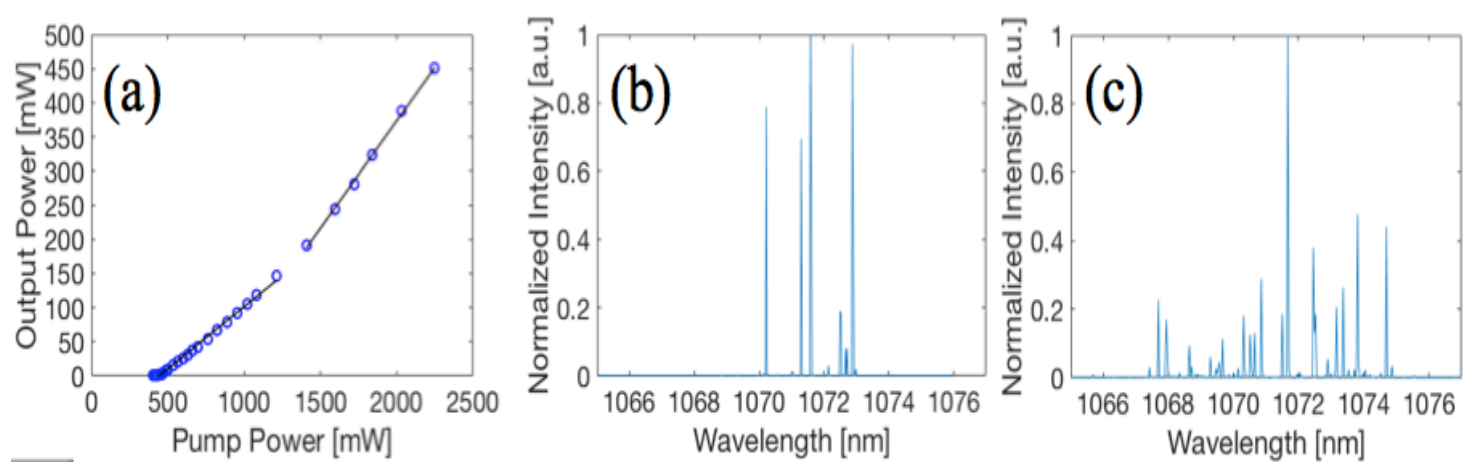

Figure 1.6: Output measurements for the free-running SBS lasing with the bare-fiber cavity configuration. (a) Output power as a function of pump power (b) Spectral output for pump power $954 \mathrm{~mW}$ (c) Spectral output for pump power $1.72 \mathrm{~W}$. 

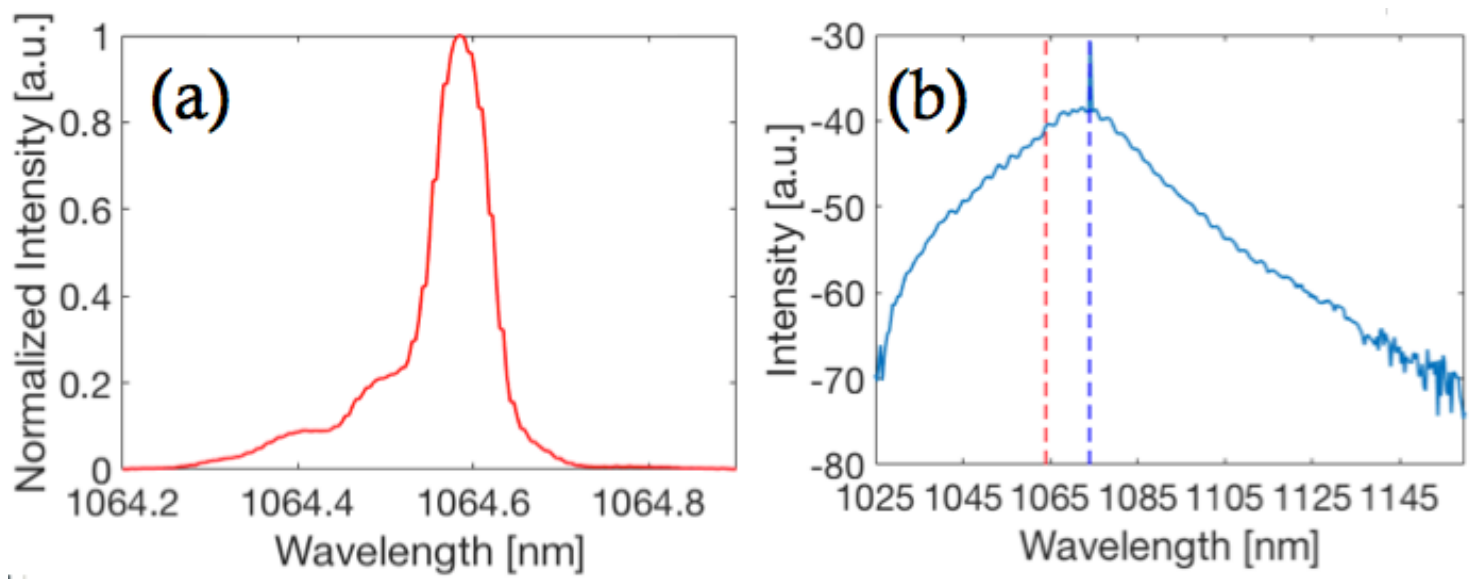

Figure 1.7: (a) Injection spectrum (b) ASE spectrum slightly above threshold, $1064 \mathrm{~nm}$ (dotted red), $1072 \mathrm{~nm}$ (dotted blue).

(a)

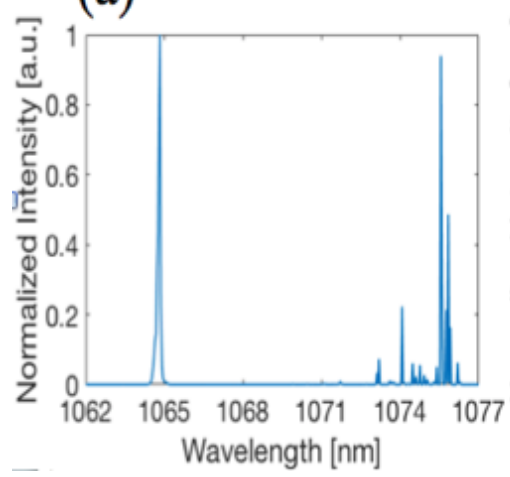

(b)

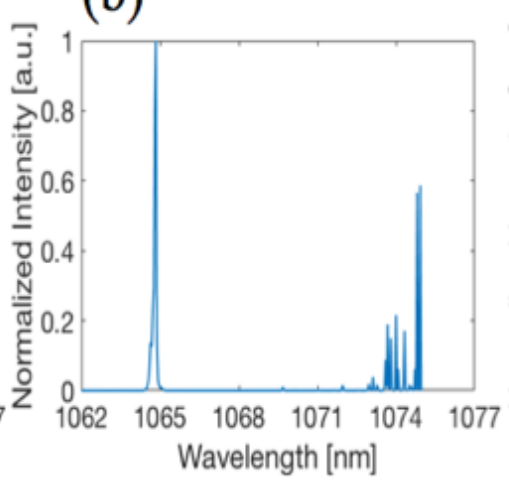

(c)

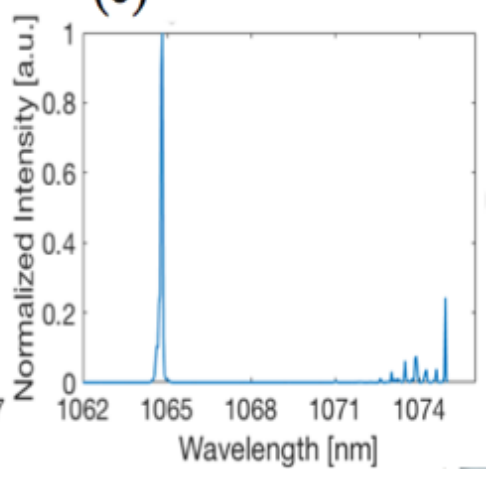

Figure 1.8: Output laser spectra with $\mathrm{CW}$ injection and partial mirror with varying losses:

(a) maximum loss (b) moderate loss (c) minimum loss. 

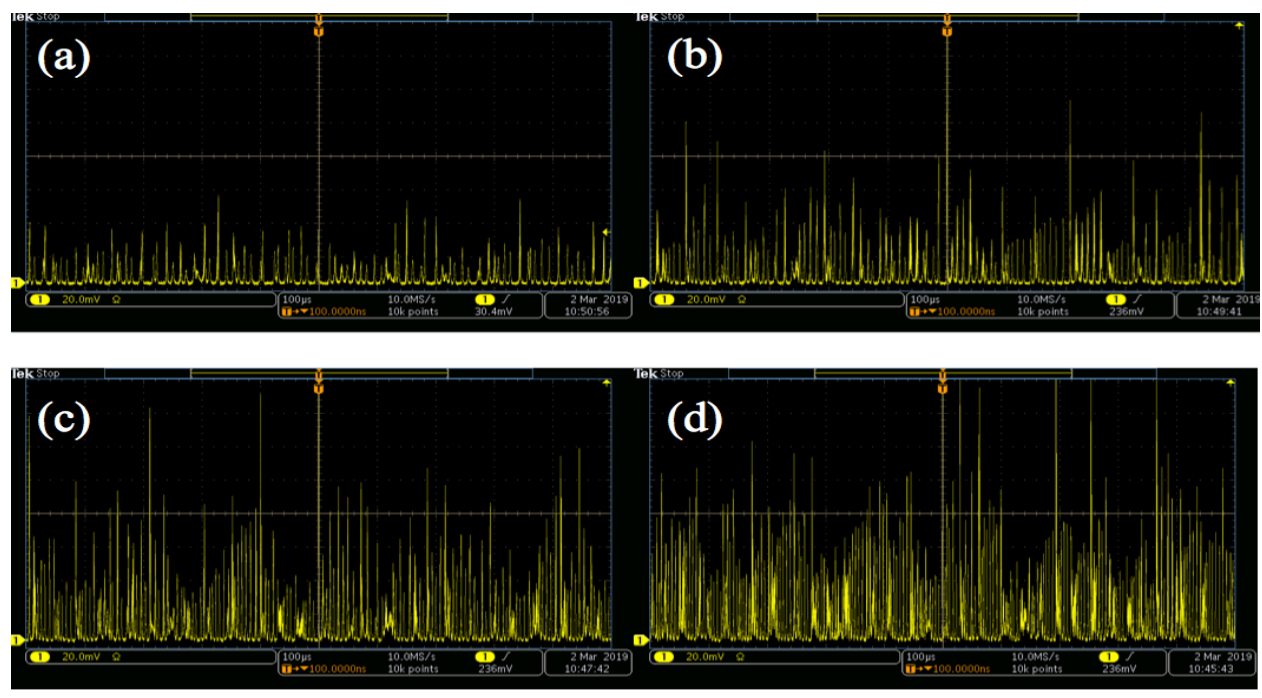

Figure 1.9: Oscilloscope traces in the Littrow configuration: (a) Pump power $\sim 824 \mathrm{~mW}$ (b) Pump power $\sim 1.08 \mathrm{~W}$ (c) Pump power $\sim 1.47 \mathrm{~W}$ (d) Pump power $\sim 1.84 \mathrm{~W}$.
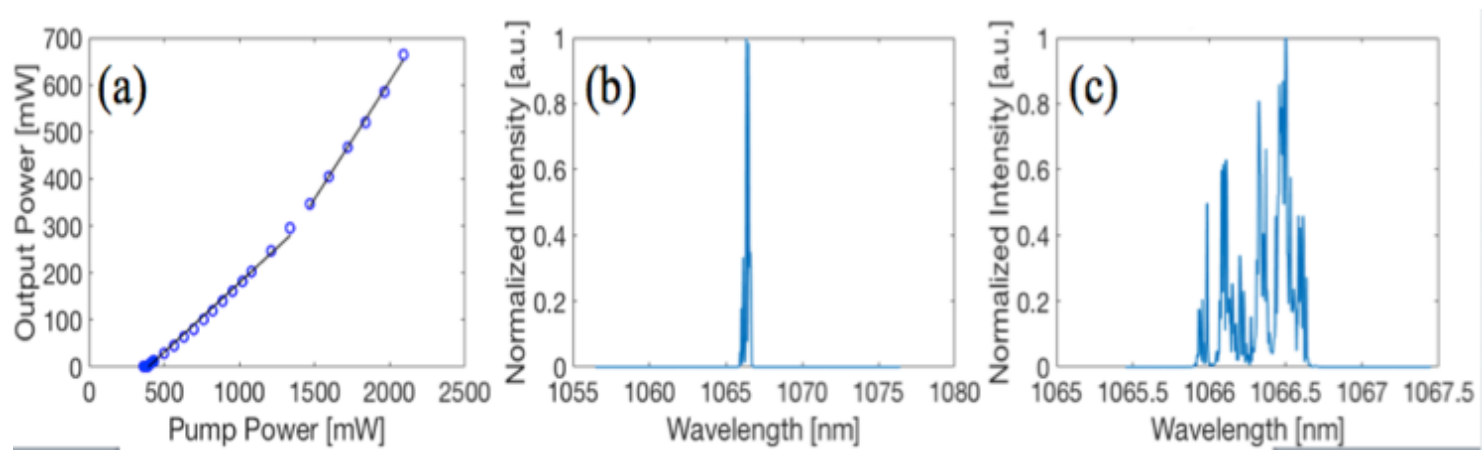

Figure 1.10: Output measurements for the Littrow configuration configuration: (a) Output power as a function of pump power (b) Output spectrum wide span at pump power $2.1 \mathrm{~W}$ (c) Output spectrum narrow span at pump power $2.1 \mathrm{~W}$. 

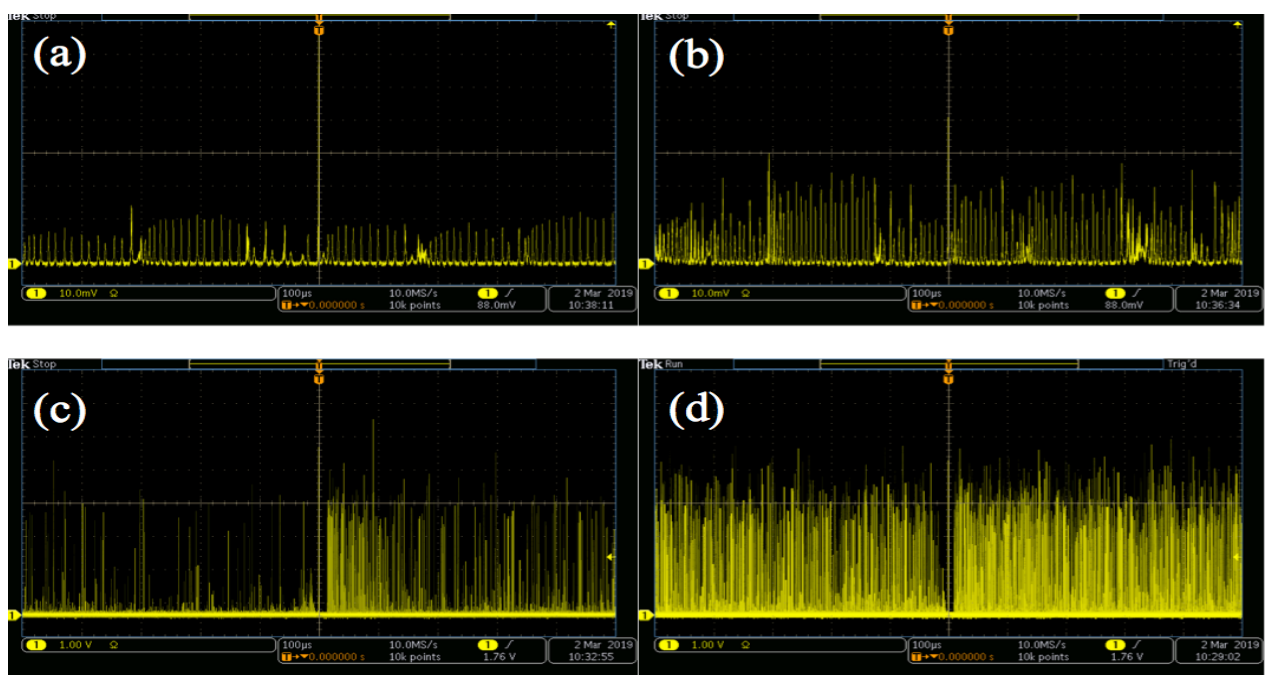

Figure 1.11: Oscilloscope traces for Littrow configuration with cavity losses: (a) Pump power $\sim 824 \mathrm{~mW}$ (b) Pump power $\sim 1.08 \mathrm{~W}$ (c) Pump power $\sim 1.47 \mathrm{~W}$ (d) Pump power $\sim 1.84 \mathrm{~W}$.
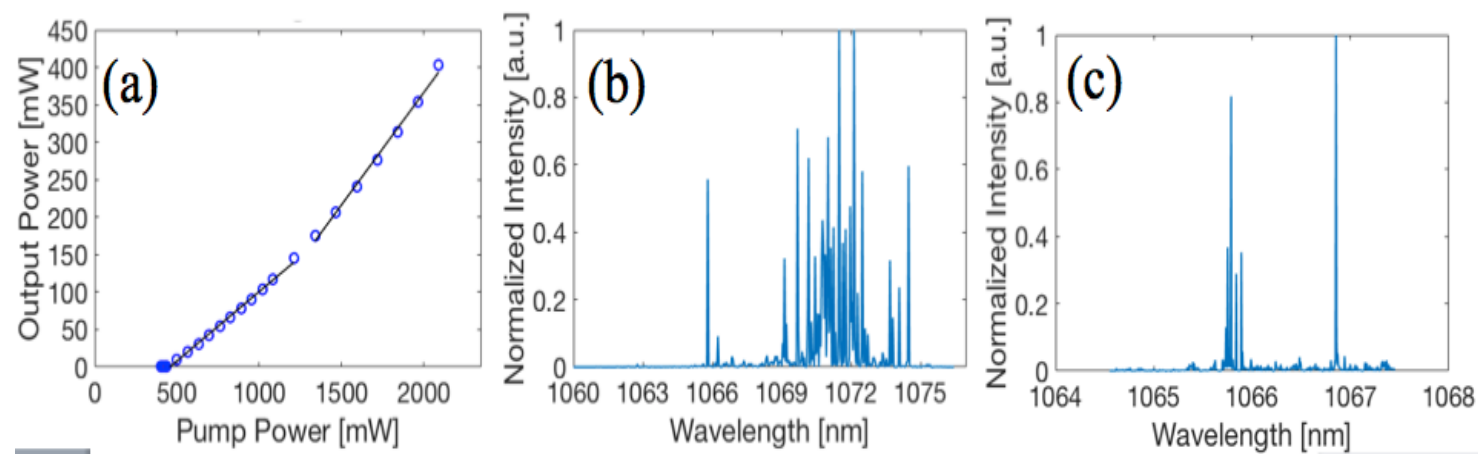

Figure 1.12: Output measurements for the free-running SBS with grating with cavity losses. (a) Output power as a function of pump power (b) (c) Output spectra for pump greater than threshold. 


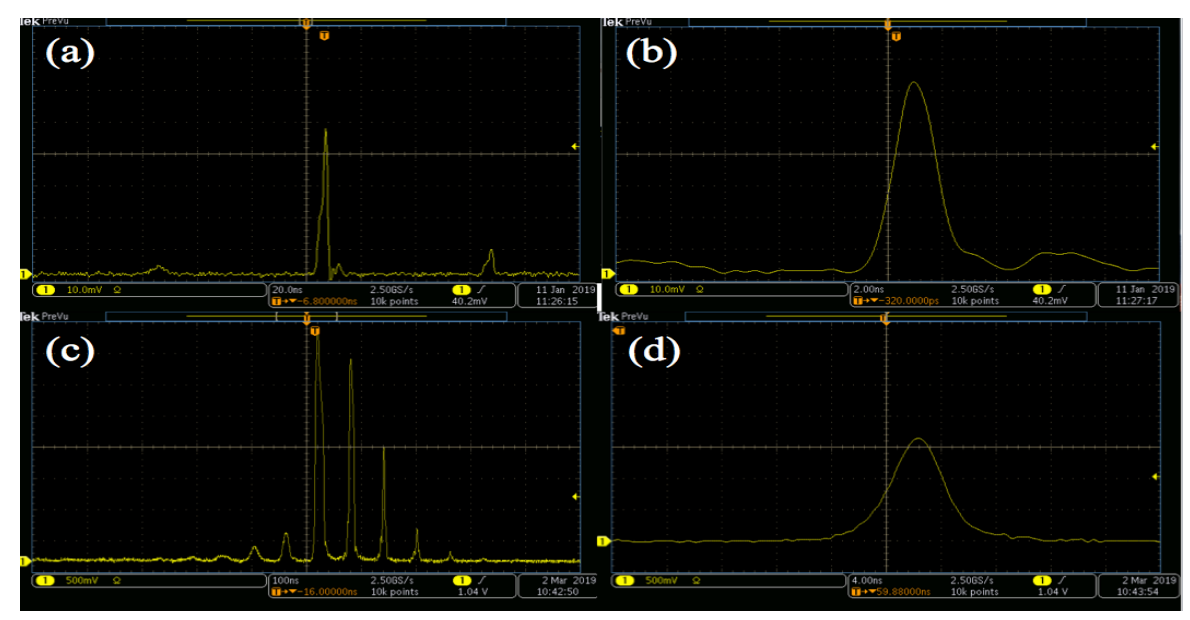

Figure 1.13: Details of the SBS single-pulse shape without injection: (a) (b) Bare-fiber termination configuration (c) (d) Littrow configuration with loss.

(a)

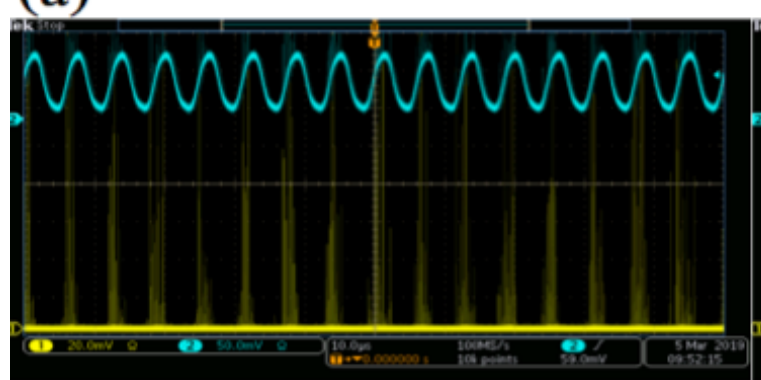

(c)



(b)

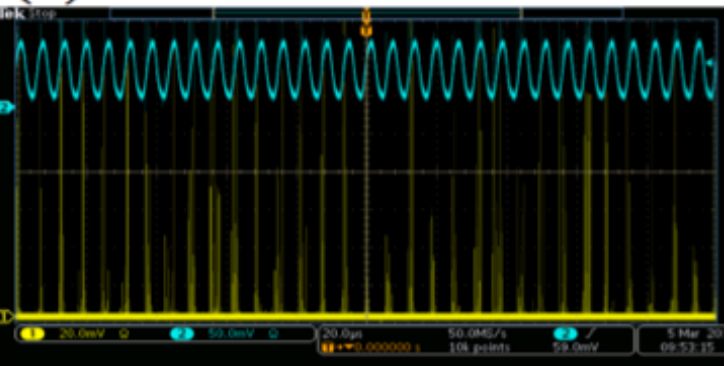

(d)

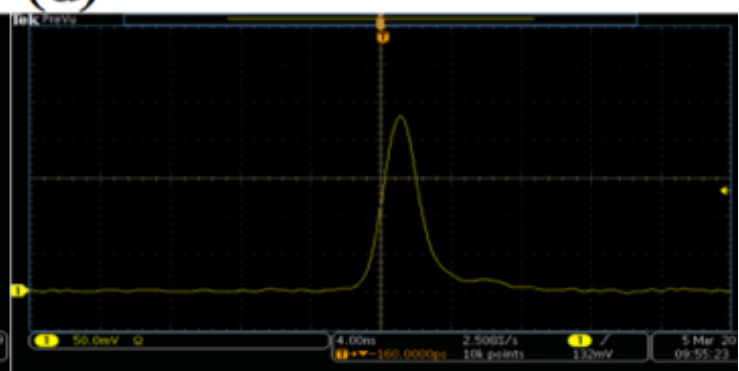

Figure 1.14: Oscilloscope traces for sine wave modulated light injection. (a) (b) Typical output pulse train sine wave injection (blue) and SBS pulses (yellow). (c) (d) Individual SBS pulses with sine wave injection. 

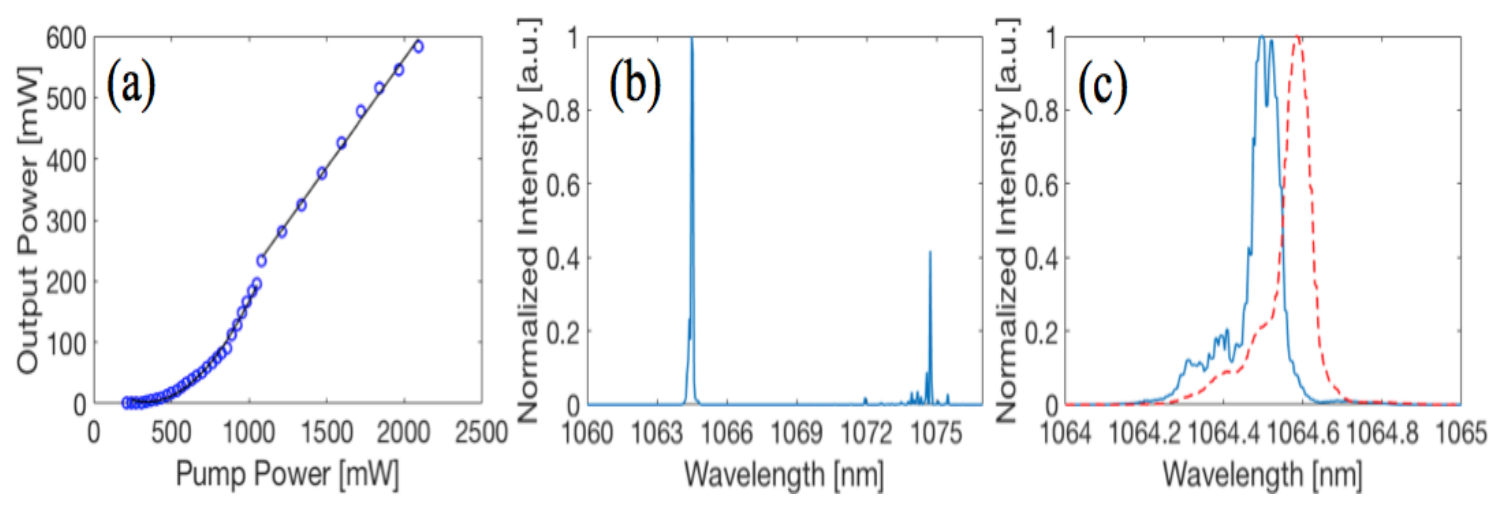

Figure 1.15: Output measurements for continuous sine wave modulated light injection.

(a) Output power as a function of pump power (b) (c) Output spectrum (blue) injection spectrum (dotted red).

(a)

(b)

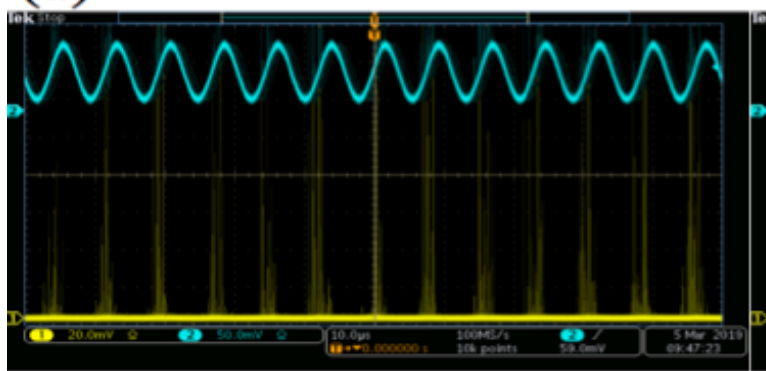

(c)

(d)
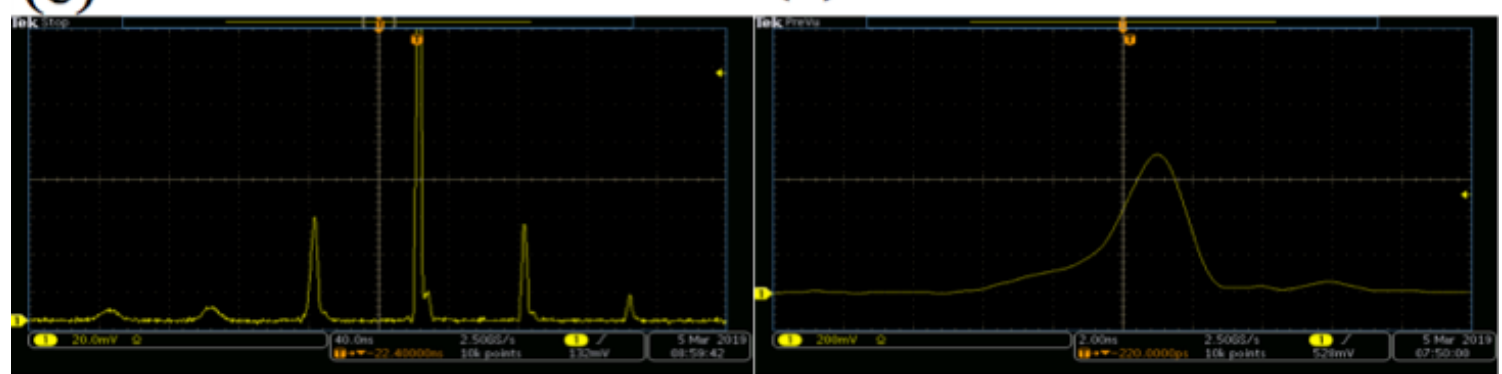

Figure 1.16: Oscilloscope traces for the continuous triangular wave modulated light injection. (a) (b) Typical pulse train. (c) (d) Single SBS pulse with injection. 

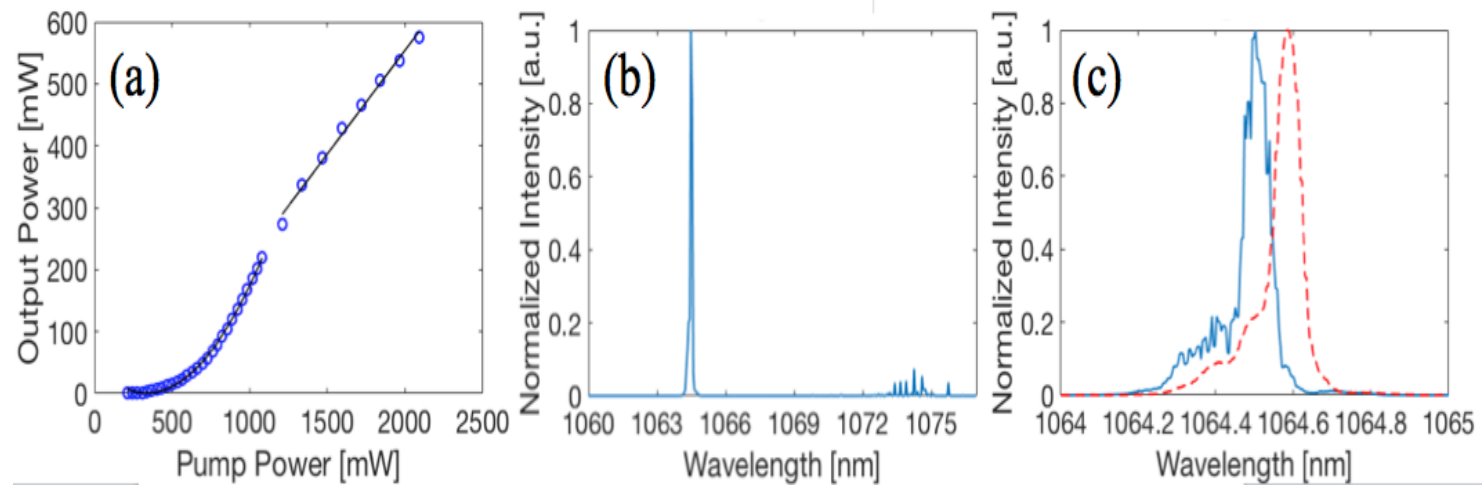

Figure 1.17: Output measurements for the triangular wave modulated light injection. (a) Output power as a function of pump power (b) (c) Output spectrum (blue) and injection spectrum (dotted red).

(a)
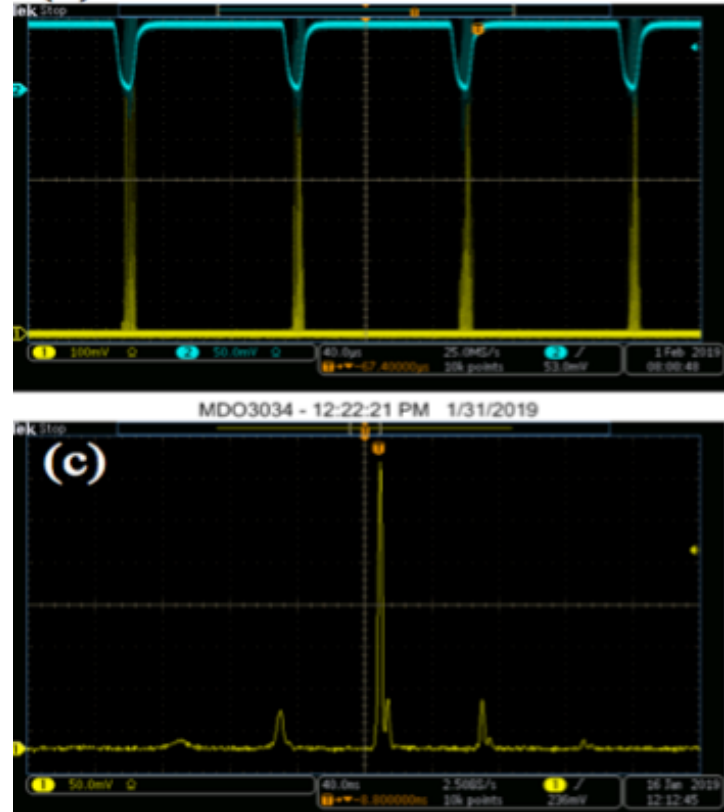

(b)
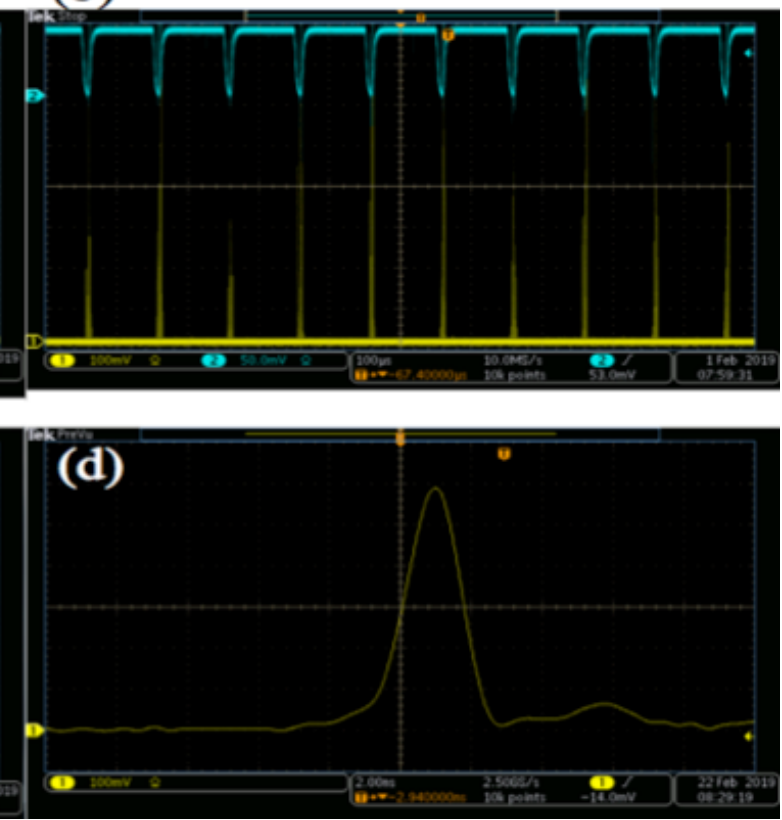

Figure 1.18: Oscilloscope traces for half-sine modulated light injection. (a) (b) Typical pulse train with injection (blue) and SBS pulses (yellow) (c) (d) Individual SBS pulses. 

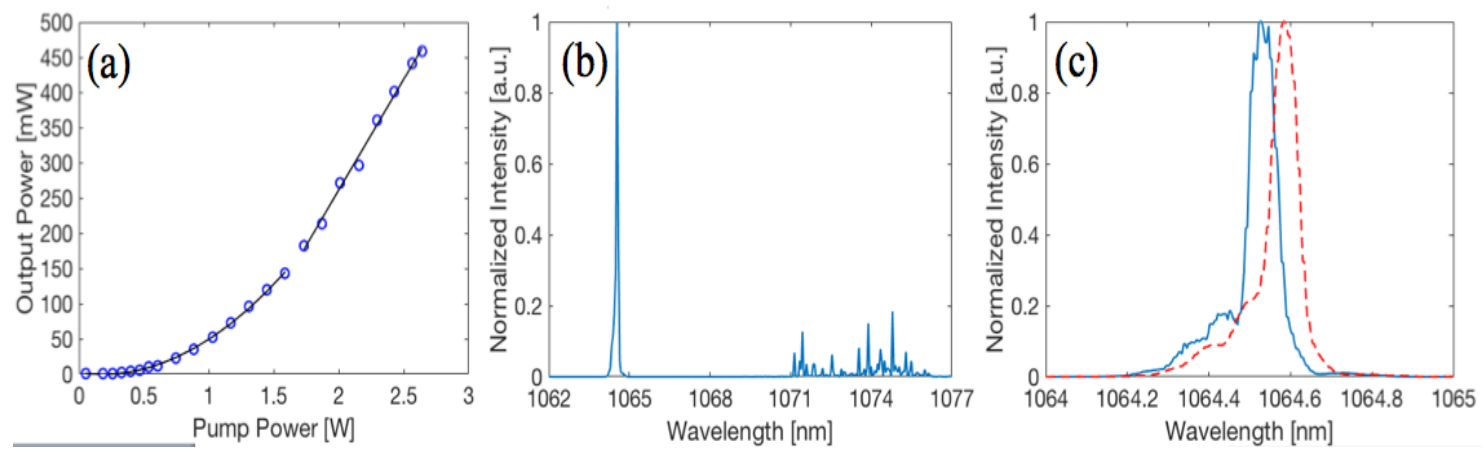

Figure 1.19: Output measurements for the half-sine pulse modulated light injection. (a) Output power as a function of pump power (b) (c) Output spectrum (blue) and injection spectrum (dotted red). 


\section{CHAPTER 2}

Published in American Institute of Physics (AIP) Advances, February 2016

\section{Resolving fine spectral features in lattice vibrational modes using femtosecond coherent spectroscopy}

Adam Card, Mohammed Mokim, and Feruz Ganikhanov

Department of Physics, University of Rhode Island, 2 Lippitt Road, Kingston, RI, 02881.

Corresponding Author: $\quad$ Feruz Ganikhanov, Ph. D.

Department of Physics

University of Rhode Island

East Hall, 2 Lippitt Road

Kingston, RI, 02881, USA

Phone: (401) 874-2633

Email address: fganikhanov@uri.edu 
Precise information on fine structure and decay of Raman active modes is essential from both fundamental and device applications point of views. Time-domain studies provide direct information on decay and dephasing processes for vibrational modes and, for solid-state media, provide most valuable information as concerned parametric phonon interaction due to deformation potential anharmonicity. In frequency domain, dispersion of the corresponding nonlinear optical susceptibility is an essential characteristic in order to get an insight into physics of intra- and interatomic groups interactions. In this paper we focus on an important nonlinear optical gain material that is used both as intracavity and external gain material in multi-wavelength laser devices. The attention has recently grown due to possible applications of efficient frequency converters in generating phase-locked frequency combs for attosecond waveform generation. Potassium titanyl orthophosphate $\mathrm{KTiOPO}_{4}(\mathrm{KTP})$ is a widely known optical material that is particularly attractive for nonlinear optical applications. Because of its high nonlinear optical coefficient and its optical and mechanical stability, the crystal is used in laser sources as an optical frequency converter. Its large electro-optic coefficient, low dielectric constant and ion exchange properties also make it suitable for electro-optic [1] and waveguided laser devices [2]. The crystal was previously shown to be an efficient source for multi-wavelength pulse generation via stimulated Raman scattering (SRS) $[3,4]$ or as a combination of SRS and efficient second order frequency conversion [5]. Renewed interest came with recent SRS experiments on high-frequency crystal vibrations that promised a pathway towards a solid-state sub-optical-cycle waveform source [6-8]. In other words, materials with high second and third order nonlinearity associated with several Raman active vibrations at high frequency range are of interest from the 
standpoint of generating a frequency comb that would ultimately support attosecond waveforms [9]. Knowledge of key properties of lattice vibrations is thus important in the light of the applications of this material as a nonlinear gain (of both second and third order) medium.

KTP's vibrational spectra are quite complex. The spectra consist of about 100 Raman active peaks as a result of the crystal's multiatomic unit cell. The complexity makes it difficult to perform comprehensive and unambiguous phonon line assignment, to precisely measure bandwidth and separation of individual Raman active peaks, as well as to estimate Raman cross-section for each individual phonon line. Even though the material has been known for more than three decades, detailed spectroscopic studies on its Raman active vibrations are relatively scarce [10-12]. The performed studies helped to elucidate contributions to Raman and infrared spectra from major atomic units within the primitive cell, as represented by $\mathrm{TiO}_{6}$ octahedra and $\mathrm{PO}_{4}$ tethrahedra. Also, important details concerning line assignments and their major characteristics were provided by the studies. However, the information ultimately proved to be contradictory and detailed spectral features of some peaks were not provided by these experiments. In particular, the first comprehensive Raman study of KTP [10] assigned peaks at around $818 \mathrm{~cm}^{-1}$ to the $v_{1}$ symmetric stretching mode within slightly distorted $\mathrm{PO}_{4}$ tetrahedra simply due to the fact that the line does not show up in the infrared reflectivity spectra. There are either two or three components spaced apart by approximately $16-40 \mathrm{~cm}^{-1}$ with linewidths within the $15-25 \mathrm{~cm}^{-1}$ range, depending on the vibrational mode symmetry. Another study characterized Raman peaks in the vicinity of $800 \mathrm{~cm}^{-1}$ as most likely belonging to $\mathrm{TiO}_{6}$ octahedra vibrational modes of different symmetry, providing mode separations within 
$11-38 \mathrm{~cm}^{-1}$ and the linewidth range of $9.2-16.4 \mathrm{~cm}^{-1}$ [11]. A study that followed later stated that the Raman line detected at $\sim 830 \mathrm{~cm}^{-1}$ is an intergroup (Ti-O-P) vibration, but provided no details on the detected linewidths and separations for the different peaks [12].

At room temperature, the Raman spectroscopy of KTP has also been investigated from 10 to $1400 \mathrm{~cm}^{-1}$ [13-14] and also studied as a function of high pressure revealing the existence of two additional phase transitions near the critical pressures of 5.5 and $10 \mathrm{GPa}$ [15]. Temperature dependent Raman scattering were studied [16-17] and found that no phonon mode coalesces to central peak near $T_{c}$ and reported it as a sign of damped soft mode [16]. A study on polarized Raman spectra showed strongest phonon line located at $234 \mathrm{~cm}^{-1}$ [18]. It is worth mentioning that no experimental or theoretical study can be found which addresses phonon dispersion properties or mechanisms for phonon line decay. As was mentioned above, there is a motivation for a more detailed characterization of phonon vibrations in the material in the light of a search for an efficient solid-state media for a sub-optical-waveform source. Indeed, the crystal possesses several high-scattering cross-section phonon modes within energy range of $200-1000 \mathrm{~cm}^{-1}$. The modes are conveniently spaced apart so that generation of a frequency comb, via SRS with intrinsically phase-locked spectral components, would provide multi-octave bandwidth to support sub-femtosecond pulses.

In this work, we present data on the decay of some of the KTP crystal phonon modes within $640-850 \mathrm{~cm}^{-1}$. We reveal the fine structure of the vibrations by retrieving the vibrational system's response function and Raman spectra. Our data provide details on the crystal's complex vibrational spectra supported by important quantitative results. 
The data obtained for an unassigned vibrational mode at $820 \mathrm{~cm}^{-1}$ supports the conclusion that the modes decay noticeably slower when compared to high-frequency modes originating from vibrations of the main $\mathrm{TiO}_{6}$ or $\mathrm{PO}_{4}$ atomic groups. We attempt to explain our linewidth results within the framework of parametric phonon interaction due to the deformation potential anharmonicity.

Time-domain CARS spectroscopy is a valuable tool that enables probing the dynamics of elementary excitations in condensed matter. This technique monitors in time a degree of coherence within the lattice or molecular vibrations created by two ultrashort optical pulses at an earlier moment of time. Tracing the net coherence provides information on characteristic relaxation and dephasing processes. In our studies, we employed three-color CARS geometry with widely tunable $110-150$ fs pulses $[19,20]$. The experimental set up is schematically shown in Figure 2.1. The two pulses that are needed to coherently drive lattice vibrations within a sample's macroscopic volume are provided by synchronously pumped optical parametric oscillators (OPOs) running at 76 MHz. The OPOs utilize high parametric gain periodically poled lithium tantalate (PPSLT) crystals. The OPOs were simultaneously pumped by a split output of a highpower mode-locked Ti:sapphire oscillator tuned to $765 \mathrm{~nm}$. Detailed OPO characteristics and performance were reported in our recent publications [21,22]. The OPOs with pulsed outputs at $970-1020 \mathrm{~nm}$ and $1050-1100 \mathrm{~nm}$, served to coherently drive lattice vibrations with energies within $600-990 \mathrm{~cm}^{-1}$. Another small part of the Ti:sapphire oscillator was delayed and served as a probe pulse. All of the three pulses were intrinsically synchronized, made to overlap in space, and focused by a high numerical aperture (NA 1.25) objective lens. In the detection arm, we used a high numerical aperture 
(NA 0.9) condenser followed by a diffraction grating and a set of bandpass filters. This permitted efficient detection of the signal of interest on the background of other signals generated within the focal volume. A photomultiplier tube (PMT) with high gain and quantum efficiency (Hamamatsu model \#R10699) was used to detect anti-Stokes signal photons at selected wavelengths. The PMT current output was digitized by a high-speed data acquisition card. Using this experimental arrangement, we can routinely detect CARS signals versus probe pulse delay times within five decades. The corresponding total power on the sample from the three beams does not exceed $15-20 \mathrm{~mW}$. Other details and characteristics of the set up are described in our most recent work [20]. Figure 2.2 (a) demonstrates sensitivity and the attainable time resolution using the experimental arrangement. In addition, using theoretical algorithms and owing to the experiment's great sensitivity, we can retrieve the vibrational system's response function and Raman spectra for several vibrational modes. The flux-grown KTP crystal used in the experiment was cut at $\phi=40^{\circ}$ and $\theta=90^{\circ}$. Polarizations of all the three beams were made parallel and aligned in XY-plane of the crystal. Thus, technically, all the four symmetry tensor components $[10,11]$ are involved in Raman mode excitation and scattering processes during CARS.

Lattice dynamics in condensed matter is modeled as time-dependent behavior regarding the expectation value of molecular/atomic displacement amplitude under a driving force. This driving force consists of a pair of pulsed fields with an optical frequency difference matching the energy of vibration quanta [23-25]. Quantitatively, the scattering CARS signal at anti-Stokes frequency in the time-domain $\left(S_{a s}\left(t_{d}\right)\right)$ can be expressed as the following: 


$$
S_{a s}\left(t_{d}\right)=\zeta_{0} \int_{-\infty}^{\infty}|\vartheta(t)|^{2} \varepsilon_{p r}^{2}\left(t-t_{d}\right) d t
$$

In the above equation, $\vartheta(t)$ and $\varepsilon(t)$ are normalized time-dependent envelopes for atomic displacement amplitude and probe pulse, respectively. This also implies that $\zeta_{0}$ represents detected anti-Stokes signal at a zero delay. In Equation (1), the time-domain CARS signal (measured experimentally) is a function of a variable time delay $t_{d}$. The delay time value corresponds to the time delayed probe pulse in the 3-color CARS geometry. As the time delay value increases from zero, the time-domain CARS signal reveals intrinsic dephasing properties of coherently excited Raman vibrational modes.

The ensemble averaged displacement amplitude is negligible well before the arrival of the driving pulse pair and one can find a solution for the dynamics equation in the form of a correlation integral in the time-domain:

$$
\vartheta(t)=\int_{-\infty}^{\infty} g\left(t^{\prime}-t\right) \varepsilon_{1}\left(t^{\prime}\right) \varepsilon_{2}\left(t^{\prime}\right) d t^{\prime}
$$

In the equation above, $g(t)$ represents the response function of the corresponding vibrational system to $\delta$-pulsed driving fields coupled with normalized time-dependent envelopes for the driving pulse amplitudes. The above equation represents the macroscopic coherently excited vibrational amplitude in the time-domain. Once coherently excited by the driving pulses, vibrating molecules/lattice atoms corresponding to a Raman vibrational mode are forced to have the same phase. The stimulated process excites the vibrational system coherently (i.e. the molecules oscillate in unison within the excited volume). This uniform phase value then decays in time due to various dephasing properties. Equations (1) and (2) are of Fredholm type-I and can be solved using the 
Fourier transform method [26]. This is ensured by the correlation integral theorem and the fact that spectra and/or envelopes of $\varepsilon_{1}$, $\varepsilon_{2}$, and $\varepsilon_{p r}$ pulses are known and can be measured. In the case when $\vartheta(t)$ is a real function, the response function $g(t)$ and its Fourier transform can be ultimately obtained. The condition holds true for many types of vibrational systems that do not involve diffusional phase shifting events. As a consequence, precise spectra and fine features in the vicinity of Raman active vibrations can be effectively resolved. Figure 2.2 (a) shows an experimentally measured CARS signal versus delay time in the KTP crystal (open circles) under conditions that favor a coherently driven high-frequency $\left(21.3 \mathrm{THz}\right.$ or $\left.\sim 710 \mathrm{~cm}^{-1}\right)$ Raman mode. For this case, the OPO wavelengths were tuned to $1020 \mathrm{~nm}$ and $1098 \mathrm{~nm}$ respectively. The coherently driven Raman mode belongs to a main symmetric Ti-O bond stretching vibrations $\left(v_{l}\left(A_{l g}\right)\right)$ within the distorted $\mathrm{TiO}_{6}$ octahedra. The vibration has a relatively high damping rate so that the corresponding CARS signal decay time ( $250 \mathrm{fs})$ is comparable with pulse widths used in our experiments. The crystal is of high quality and the only mechanism that results in the CARS signal decreasing versus time delay, is a decay of the coherently driven phonon into phonons of lower energy. From the measurement, a crude estimate can be made for the phonon lifetime ( $T_{l} \cong 495 \mathrm{fs}$ ) and the corresponding phonon line bandwidth in $\left(\Delta v=1 / \pi c T_{l} \cong 21 \mathrm{~cm}^{-1}\right)$. A more rigorous analysis that concerns spectral domain information retrieved from the time-domain data is needed. For this particular case, the excitation and probe pulses cannot be considered as $\delta$-functions $\left(t_{p} \sim 3 \times T_{I}\right)$ and an approach reported earlier by our group, described in Ref. [27], yields in somewhat distorted spectral data. Thus, equations above need to be solved in order to retrieve Raman spectra along with the dispersion of the real part of the associated resonant third 
order nonlinearity $\left(\chi^{(3)}(\omega)\right)$. The Fourier transform $\left(S_{a s}(\omega)\right)$ of the measured timedependent CARS signal is a first step in solving the equations. The corresponding result is shown in Figure 2.2 (b). The spectrum is smooth, as high-frequency noise in the timedomain CARS signal has a relatively low spectral power density and is not visible on linear scale. The main characteristic of the spectrum is a broadband and high-intensity pedestal, associated with ultrafast signal rise-time. The pedestal masks a narrower spectral feature. The latter may reflect a slower decay rate due to the phonon decay process mentioned above. Knowing the measured probe pulse spectrum, $\left(I_{p r}(\omega)=F T\left(\varepsilon_{p r}{ }^{2}(t)\right)\right.$, and applying the inverse Fourier transform operation, allows the collection of time-domain data on the coherent displacement amplitude $(\vartheta(t))$ at $|\vartheta(t)|^{2}=F T^{-1}\left(\frac{s_{a s}(\omega)}{I_{p r}(\omega)}\right)$. Further, having $\varepsilon_{1}(\omega), \varepsilon_{2}(\omega)$ available from OPO pulse autocorrelation and spectral measurements and a Fourier transform of $\vartheta(t)$, one can arrive to resonant third order nonlinearity $\left(\chi^{(3)}(\omega)\right)$ spectra contained in real and imaginary parts of $g(\omega)$. Figure 2.2 (d) shows the retrieved coherent amplitude function $(\vartheta(t))$ and the real and imaginary part of the resonant third order nonlinearity in the vicinity of the coherently driven $v_{l}\left(A_{l g}\right)$-phonon mode. The main peak's asymmetry is caused by the presence of two components with relative amplitude ratio of 26:11, bandwidths (FWHM) of $17.2 \pm 0.7$ and $24 \pm 1.2 \mathrm{~cm}^{-1}$ and an energy separation of $16 \mathrm{~cm}^{-1}$ for the doublet. The difference in the bandwidths is explained by different damping rates for in-plane and along long axis vibrations within the $\mathrm{TiO}_{6}$ octahedron [10]. A third component is also pronounced in the spectra with a position shifted to lower energies by $65 \mathrm{~cm}^{-1}$. This mode has a different symmetry and represents $v_{2}\left(E_{g}\right)$ anti-phase stretching vibration within $\mathrm{TiO}_{6}$ octahedra. The peak can be better resolved under condition when 
one of the OPOs is detuned to provide more efficient coherent excitation for the $v_{2}\left(E_{g}\right)$ mode. As a result, the time-dependent CARS signal exhibits a more pronounced quantum beats pattern. Using this arrangement, the spectral bandwidth of the $v_{2}\left(E_{g}\right)$ mode was determined to be $21.3 \pm 0.7 \mathrm{~cm}^{-1}$. The obtained parameters for the main $v_{l}\left(A_{l g}\right)$ doublet and for the $v_{2}\left(E_{g}\right)$ modes are in good general agreement with the referenced reports $[10,11]$. We must note, however, that consistent bandwidth and Raman shift data for the doublet components could not be found throughout Raman spectroscopy characterization studies of KTP crystal published in the past [10-18, 28-31]. The result of fitting imaginary part of the resonant third order nonlinearity (i.e. Raman spectrum) using Lorentz-shaped multi-peak curves is also shown in Fig. 2.2 (d) by solid lines with individual line details provided in the figure caption.

An unassigned phonon mode centered at $\sim 820 \mathrm{~cm}^{-1}$ is perhaps a better example of the complex nature of Raman active vibrations in the crystal. Figure 2.3 (a) shows a CARS transient obtained for this case. The overall signal behavior shows quantum beats of at least two spectral components on the background of characteristic exponential decay with a noticeably longer time constant than for the $v_{l}\left(A_{l g}\right)$ mode. Modeling of the timedomain behavior of CARS signals and the fitting of the experimental data require certain assumptions and use of multi-parametric fitting algorithms that do not have global minima. We have instead analyzed the obtained data by the approach that was outlined above. Fourier analysis of the signal unequivocally reveals a presence of a strong nonresonant signal on the background of weaker contributions from the clustered phonon mode. This proves the fact that the mode is noticeably weaker in its intensity when compared to the $v_{l}\left(A_{l g}\right)$ and $v_{2}\left(E_{g}\right)$ modes. Retrieved Raman spectra $\left(\operatorname{Im}\left(\chi^{(3)}(\omega)\right)\right)$ and 
data for the real part of the third order nonlinearity are shown in Figures 2.3 (b) and 2.3 (c) respectively. Three spectral components are involved in this case. They have energy separations of $18 \mathrm{~cm}^{-1}$ and $46 \mathrm{~cm}^{-1}$ and the values are in relatively good agreement with spontaneous Raman scattering measurements. The latter provide the range of $20.4-29.0$ $\mathrm{cm}^{-1}$ and $34.6-39.2 \mathrm{~cm}^{-1}$ for the corresponding two parameters [11]. Spectral component bandwidths obtained from our data are $9.1 \pm 0.4 \mathrm{~cm}^{-1}, 7.5 \pm 0.6 \mathrm{~cm}^{-1}$, and $11.2 \pm 0.5 \mathrm{~cm}^{-1}$ for the three components compared to ranges of $10.2-12.6 \mathrm{~cm}^{-1}, 9.2-10.8 \mathrm{~cm}^{-1}$, and $14.0-$ $16.4 \mathrm{~cm}^{-1}$ respectively reported by spontaneous Raman spectroscopy study [11]. The spread for both parameters is dependent on particular experimental conditions (e.g. crystal axes orientations with respect to laser polarization) when different scattering tensor elements have been accessed within the measurements. And finally we report a component amplitude ratio of 46:5:31. The value is not available for comparison from spontaneous Raman spectroscopy studies Phonon line bandwidths are approximately two times narrower (i.e. the corresponding phonon decay rate is two times lower) when compared to the high frequency modes $\left(v_{l}\left(A_{1 g}\right)\right.$ and $\left.v_{2}\left(E_{g}\right)\right)$ that are stronger in Raman scattering. We explain this by the fact that the latter modes have a variety of efficient overtone or combinational phonon decay channels within either of the $\mathrm{TiO}_{6}$ or $\mathrm{PO}_{4}$ groups, resulting in lower energy vibrations. Therefore, we think, that based on the fact that the investigated $\sim 820 \mathrm{~cm}^{-1}$ mode has a significantly lower damping rate, the mode is not a vibration originating from either of the two main atomic groups and it is rather within Ti-O-P intergroup vibrations. The complex structure (i.e. presence of the triplet line) can be explained by shifted frequencies for vibrations of different symmetry within the group. Lower phonon damping rates (i.e. higher effective dephasing time $T_{2} *=T_{1}$ ) 
makes up to a certain degree for the difference in the steady state SRS gain between the relatively weak mode at $820 \mathrm{~cm}^{-1}$ and the strong $v_{l}\left(A_{l g}\right)$ and $v_{2}\left(E_{g}\right)$ vibrations. By using proper crystal orientation, it is possible to produce in SRS experiments (Stokes and antiStokes scattering) a nearly equal intensity and equidistant comb of frequencies that includes $\sim 820 \mathrm{~cm}^{-1}$ mode. The comb can be used for ultrafast waveform synthesis.

In conclusion, we have demonstrated, using femtosecond time-domain coherent anti-Stokes Raman scattering spectroscopy, a resolution of complex Raman active vibrations in KTP crystal. The Raman spectra retrieved from our measurements show several spectral components corresponding to vibrations of different symmetries with distinctly different damping rates. Relative amplitude ratio, energy shifts, and bandwidths for an unassigned optical phonon mode triplet centered at $\sim 820 \mathrm{~cm}^{-1}$ have been reported. The mode is thought to belong to vibrations in the Ti-O-P intergroup within the crystal. Results of our experiments can be used to estimate stimulated Raman gain for different vibrational modes in the crystal.

Authors acknowledge funding support from NSF (DBI-135530).

\section{References:}

${ }^{1}$ X. D. Wang, P. Basseras, R.J.D. Miller, H. Vanherzelle, Appl. Phys. Lett. 59, 519 (1991)

${ }^{2}$ Savatinova, I. Savova, E. Liarokapis, C.C. Ziling, V.V. Atuchin, M.N. Armenisek, V.M.N. Passrok, J. Phys.D: Appl. Phys. 31, 1667(1998).

${ }^{3}$ V. Pasiskevicius, A.Fragemann, F. Laurell, R. Butkus, V. Smilgevicius, A. Piskarskas, App. Phys. Lett. 82, 325(2003). 
${ }^{4}$ V. Pasiskevicius, C. Canalias, F. Laurell, , App. Phys. Lett. 88, 041110(2006).

${ }^{5}$ Y.F. Chen, Opt. Lett. 30,400 (2005).

${ }^{6}$ L.L.Losev, J. Song, J.Xia, D. Strickland, and V. Brukhanov, Opt. Lett. 27, 2100 (2002)

${ }^{7}$ M. Zhi, A. Sokolov, Opt. Lett. 32 , 2251 (2007).

${ }^{8}$ E. Matsubara, T. Sekikawa, and M. Yamashita, App. Phys. Lett. 92, 071104 (2008).

${ }^{9}$ S. Baker, I. Walmsley, J.W.G. Tisch, and J.P.Marangos, Nature Photonics 5, 664 (2011).

${ }^{10}$ G. E. Kugel, F. Brehatt, B. Wyncket, M. D. Fontanat, G. Marniers, C. CarabatosNedelect, J. Mangin, J. Phys. C: Solid State Phys. 21, 5565(1988).

${ }^{11}$ G.H. Watson, J. Raman Spectrosc. 22, 705 (1991).

${ }^{12}$ K. Vivekanandan, S. Setvasekarapandian, P. Kolandaivel, M.T. Sebastian, S. Suma, Mater. Chem. Phys. 49, 204 (1997).

${ }^{13}$ G.A Massey, T.M. Loehr, L.J. Willis, J.C. Johnson, Appl. Optics 19, 4136 (1980).

${ }^{14}$ V.M Garmash, D.N. Govorun, P.A. Korotkov, V.V Obukhovskii, N.I. Pavlova, I.S Rez, Opt. Spectrosc. 58, 424(1985).

${ }^{15}$ G.A. Kourouklis, A. Jayaraman, A.A. Ballamn, Solid State Commun. 62, 379 (1987).

${ }^{16}$ Y.K. Voronki. V.A. Dyahov, A.B. Kudryavtsev, V.V. Osiko, A.A. Sobol, E.V. Sorokin,Sov.Phys.-Solid State 31, 1736 (1989).

${ }^{17}$ S.Fursawa, H. Hayasi, Y. Ishibashi, A. Miyamoto, T.Sasaki, J.Phys.Soc.Jpn. 60, 2470 (1991).

${ }^{18}$ C.S. Tu, A.R. Guo, R. Tao, R.S. Katiyar, R. Guo, A.S. Bhalla, J. Appl. Phys. 79, 3235 (1996).

${ }^{19}$ S.Yang, R.Wysolmerski, and F. Ganikhanov, Opt. Lett. 36, 3849(2011).

${ }^{20}$ S.Yang, S.Adhikari, L. Zhang, R.Wysolmerski, G, Spirou, and F. Ganikhanov, Appl.

Phys B: Lasers and Optics 111, 617(2013). 
${ }^{21}$ K.Bhupathiraju, A.Seymour, F.Ganikhanov, Opt. Lett. 34, 2092 (2009).

${ }^{22}$ J.Rowley, S.Yang, F. Ganikhanov, J. Opt. Soc. Am. B 28, 1026 (2011).

${ }^{23}$ A. Laubereau, D. von der Linde, and W. Kaiser, Phys. Rev. Lett. 28, 1162 (1972).

${ }^{24}$ A. Laubereau and W. Kaiser, Rev. Mod. Phys. 50, 607 (1978).

${ }^{25}$ S. A. Malinovskaya, Opt. Lett. 33, 2245(2008).

${ }^{26}$ A.Jerry, Introduction to Integral Equations With Applications, (2d edition, John Willey and Sons, Inc., 1999)

${ }^{27}$ S. Yang, F. Ganikhanov, Opt. Lett. 38, 4754 (2013).

${ }^{28}$ Ch. Ferrer, A. Segura, M.V. Andres, V. Munoz, J. Pellicer, J. App. Phys. 79,3200 (1996).

${ }^{29}$ X. Liwen, C. Dawei, N. Hongda, Chinese Phys. Lett. 1.6,225 (1989).

${ }^{30}$ M.J. Bushiri, V.P. M. Pillai, R. Ratheesh, V.U. Nayar, J. Phys. Chem. Solids 60, 1983 (1999).

${ }^{31}$ M.J. Bushiri, V.U. Nayar, J. Nonlinear Optic. Phys. Mat. 10, 345 (2001). 


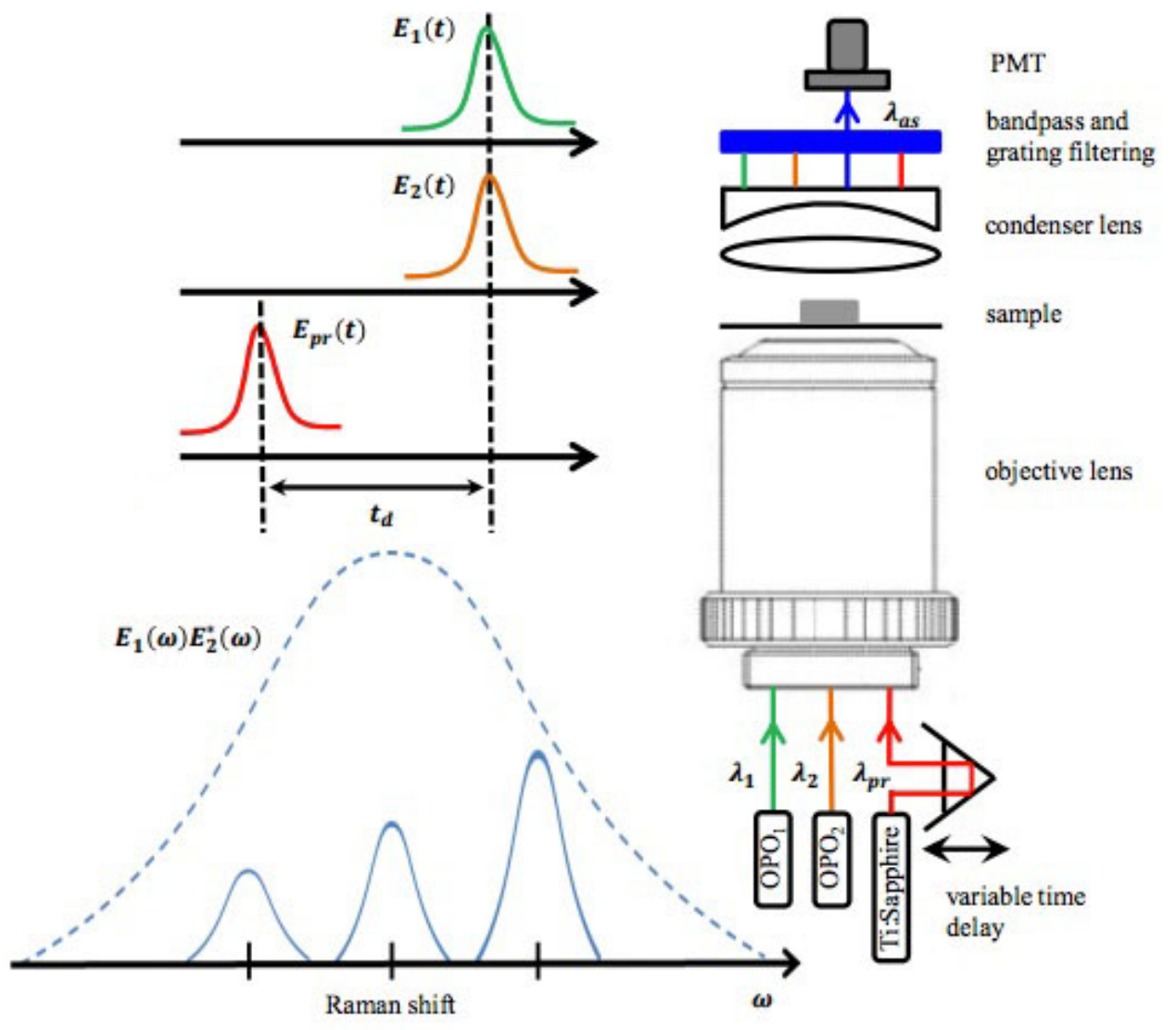

Figure 2.1: Experimental diagram and layout for three-color time-domain CARS spectroscopy experiments that employ tunable optical parametric oscillators. Pair of transform-limited 110-150 fs pulses at $\lambda_{1}$ and $\lambda_{2}$ wavelengths are used to coherently drive Raman active vibrational modes in the vicinity of corresponding $\omega_{1}-\omega_{2}$ frequency shifts. A pulse at $\lambda_{\mathrm{pr}}=765 \mathrm{~nm}$ is time delayed and probes the resulting coherent excitation at different delay times. CARS transients at anti-Stokes frequency are detected within five orders of magnitude. Polarizations of all the three beams were made parallel to each other and aligned within XY-plane of the KTP crystal under study. 

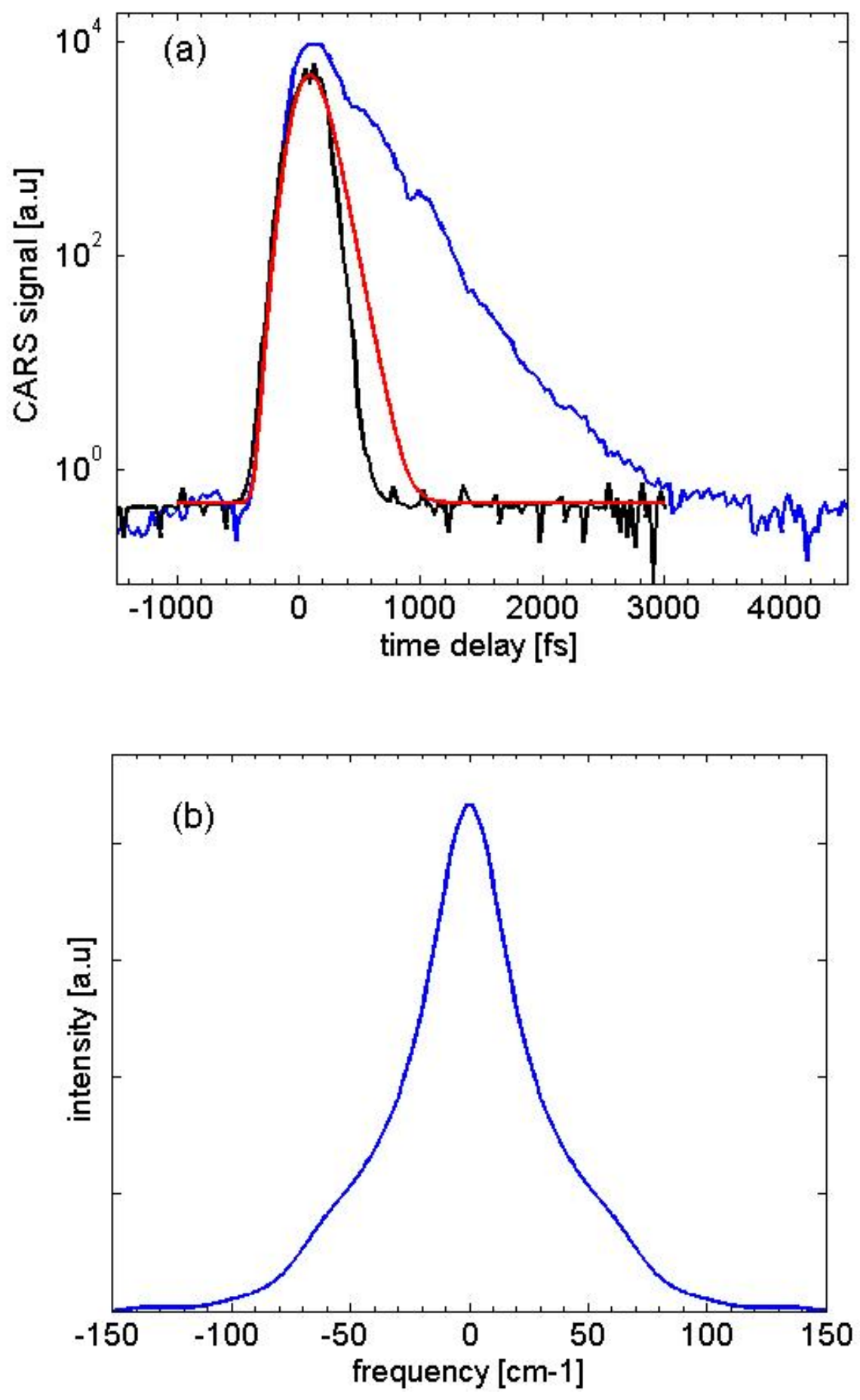

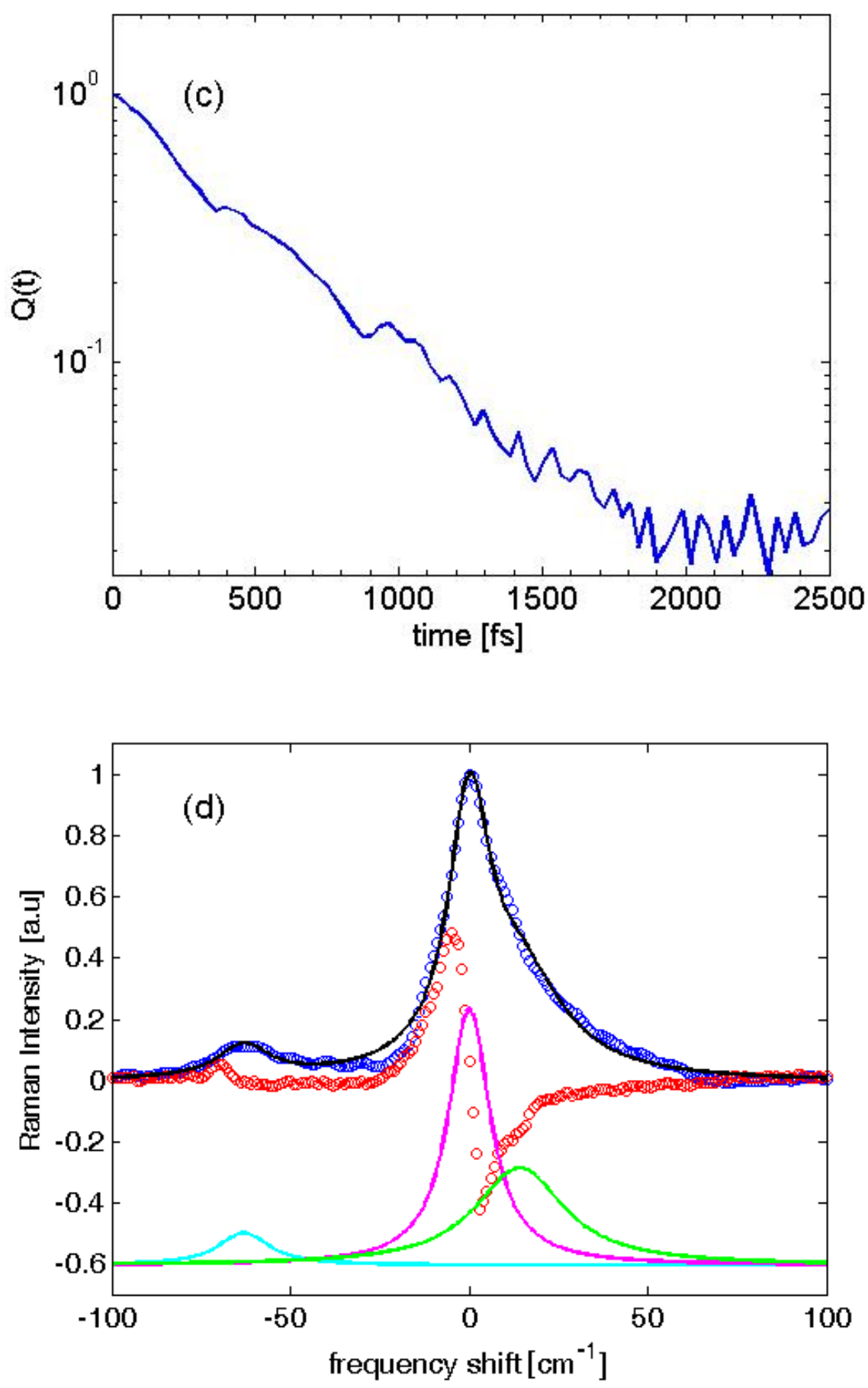

Figure 2.2: (a) Time-domain CARS system instrument response obtained in quartz glass (black curve). Simulated CARS transient for Raman active vibrational mode with dephasing time $T_{2}=150 \mathrm{fs}$ (red curve). CARS transient detected in KTP crystal under the conditions specified 
below (blue curve). The broadband OPO pulses were tuned to $1020 \mathrm{~nm}$ and $1100 \mathrm{~nm}$ center wavelengths to coherently drive the main $v_{l}\left(A_{l g}\right)$ high frequency Raman active mode at $\sim 700 \mathrm{~cm}^{-}$ ${ }^{1}$. (b) CARS signal spectrum ( $S_{a s}(\omega)$ ) showing a narrower spectral feature on the background of a broadband pedestal corresponding to the transient signal's fast rise time. (c) Time-domain response function $(g(t))$ that was obtained by solving equations (1) and (2) using Fourier transform method. (d) Dispersion of real (red open circles) and imaginary (blue open circles) parts of the resonant third order optical nonlinearity $\left(\chi^{(3)}(\omega)\right)$ in the vicinity of the coherently driven Raman active modes. The part corresponding to Raman spectra (i.e. $\operatorname{Im}\left(\chi^{(3)}(\omega)\right)$ is also shown fitted with solid black curve representing a sum of three Lorentz-shaped envelopes with bandwidths of $21 \mathrm{~cm}^{-1}, 17 \mathrm{~cm}^{-1}$, and $25 \mathrm{~cm}^{-1}$ starting from the red shifted $v_{2}\left(E_{g}\right)$-mode (cyan color peak).

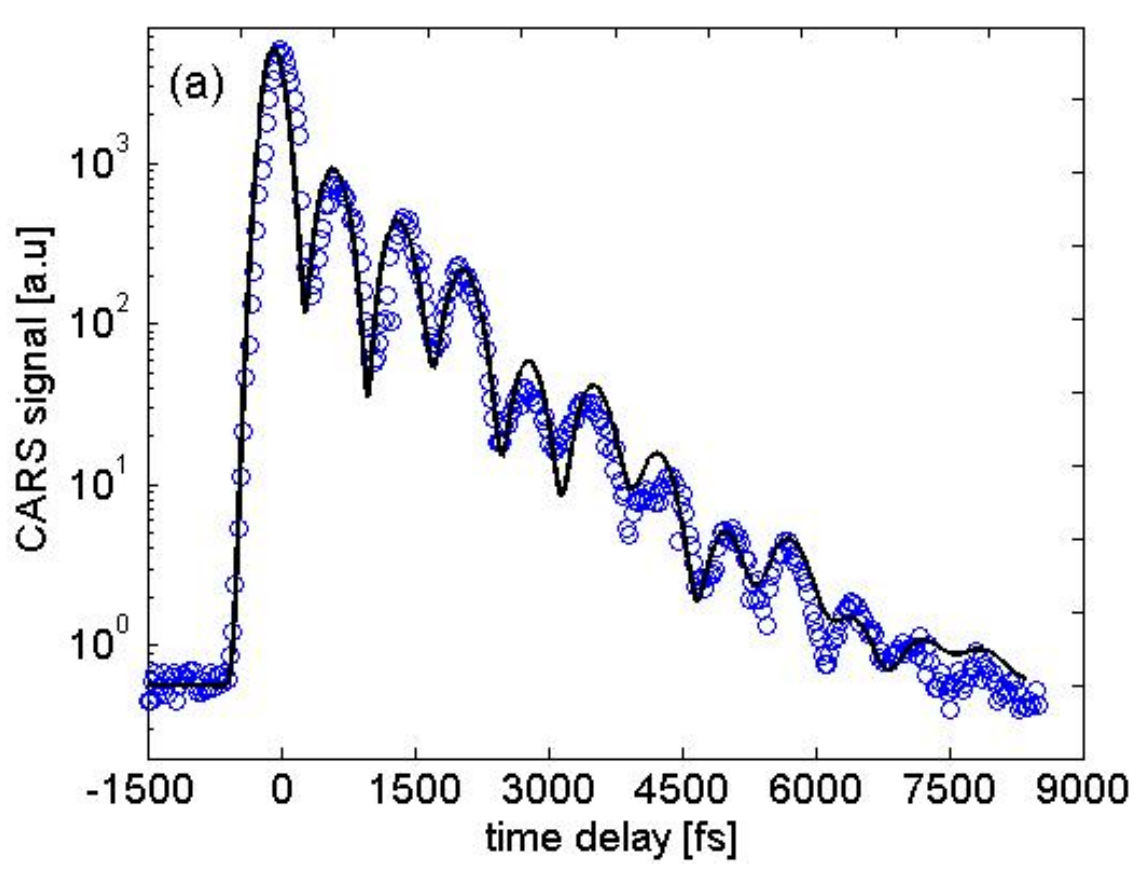



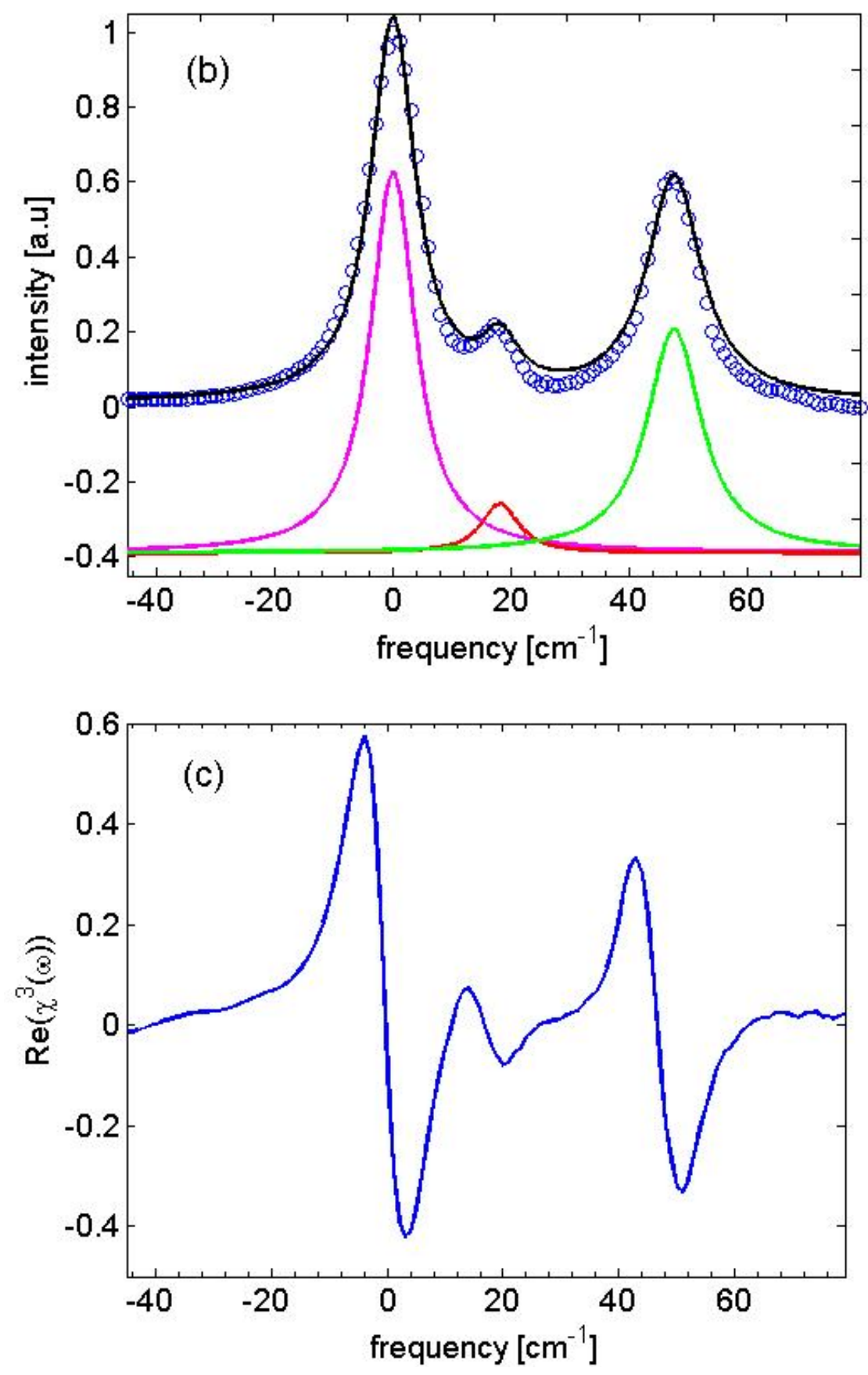

Figure 2.3: (a) CARS transient (blue circles) obtained from KTP crystal when unassigned Raman active vibrational modes at $\sim 820 \mathrm{~cm}^{-1}$ are coherently driven and probed. A proper wavelength combination was chosen for the OPOs $\left(\lambda_{1}=967 \mathrm{~nm}\right.$ and $\left.\lambda_{2}=1051 \mathrm{~nm}\right)$ so that the corresponding shift is targeted at its center. Simulated CARS signal (black curve) 
obtained under amplitude, damping rate, spectral shift parameter values for the vibrational modes using retrieved Raman spectra data. (b) Retrieved Raman spectra (blue curve), simulated Lorentz-shape curves for the three spectral components that yield in cumulative fitting curve (black). (c) Corresponding real part of the third order optical nonlinearity. 


\section{CHAPTER 3}

Published in Applied Sciences, August 2018

\section{Quantitative ultrafast spectroscopy and microscopy of traditional and soft condensed matter}

Adam Card, Mohammed Mokim, and Feruz Ganikhanov

Department of Physics, University of Rhode Island, 2 Lippitt Road,

Kingston, RI, 02881.

Corresponding Author: $\quad$ Feruz Ganikhanov, Ph. D.

Department of Physics

University of Rhode Island

East Hall, 2 Lippitt Road

Kingston, RI, 02881, USA

Phone: (401) 874-2633

Email address: fganikhanov@uri.edu 
Applications of nonlinear optics have been widely regarded as powerful tools that are capable of providing quantitative spectral information in condensed matter characterization. These applications span from plasma to solid-state materials and nanostructures, as well as to interfaces and biological media $[1,2,3,4,5]$. Raman effect based spontaneous and coherent scattering techniques are of special attention due to their selectivity and sensitivity down to a chemical bond level, and that in turn provides access to important physical mechanisms and fundamental interactions. However, in spontaneous Raman spectroscopy, reliable data on weak resonances is difficult to obtain. In the last decade, coherent Raman spectroscopy methods have been successfully demonstrated in optical microscopy $[6,7,8,9,10]$. Spectral responses with a resolution of a few $\mathrm{cm}^{-1}$ have been demonstrated in various applications that focused on the characterization of biological matter. It is worth noting that these approaches provide only dispersion for the imaginary part of the resonant optical nonlinearity. The overwhelming majority of studies are in the frequency domain, with a recent focus on novel solid-state materials $[11,12,13,14]$ and molecular/biological media $[15,16,17,18]$. The coherent Raman based microscopy techniques were recently applied to image biological tissue and cells with sub-micron spatial resolutions capable of resolving the spatial structures of key constituents when their characteristic Raman active vibrations are targeted $[19,20]$. The spontaneous Raman microscopy has been applied with a greater focus towards the analysis of specific vibrations within cells, tissues and their spectral features. Multi-line spectra were analyzed with respect to relative changes in the intensities and spectral shifts for main phonon lines, depending 
on growth techniques for solid-state materials as well as scattering geometries with respect to crystal axes $[21,22]$. For biological media, efforts were focused towards the goal of correlating those with bio-molecular alterations occurring on cellular and subcellular levels $[23,24]$. Resolving lattice or molecular vibration damping rates $\Gamma$ (or linewidths, $\Delta v=1 / \Gamma)$ and line shapes, which are ultimately key parameters in characterizing atomic/molecular bonds, has not been of specific effort primarily due to insufficient detection sensitivity and spectral resolution. The vibration damping rate is strongly influenced by unit cell structure and respective phonon properties in crystals and inter- and intra-molecular interactions in soft media. Therefore, the ability to measure Raman line shapes and sub-structures with high precision is absolutely important from the standpoint of having access to fine inter- and intra-molecular interactions and their physical mechanisms. Spontaneous Raman spectroscopy has a typical resolution of $\sim 3-7 \mathrm{~cm}^{-1}$ and often requires the application of data postprocessing algorithms to retrieve spectra. Thus, for current state-of-the-art spectral domain techniques, the ability to access critical inter- and intra- molecular interactions of key molecular groups within biological specimens is limited. Coherent Raman microscopy, which employs picosecond pulses to attain high peak powers, cannot provide a better spectral resolution due to the pulse bandwidths $\left(\sim 5-20 \mathrm{~cm}^{-1}\right)$. Additional measurement artifacts and sources of imprecision come from a need to mechanically tune laser wavelengths in point-by-point spectral measurements with an obvious adverse impact on precision. Thus, the desired precision in detecting Raman line shapes and bandwidths may not be achieved. Precise information on dispersion of the nonlinear optical susceptibility in Raman active media is essential in order to gain 
insight into the physics of intra- and inter-atomic and molecular interactions. For solid state, detailed knowledge of Raman active vibrational properties in intrinsic and doped materials, as well as in thin films, is of high value from the standpoint of identifying mechanisms that limit transport parameters which are critical for device applications. For biological media, this can become a powerful tool in detecting small spectral changes that can be translated to variations in bio-molecular composition and ultimately lead to disease diagnoses on a molecular level.

In this report, we discuss and implement a method that is applied to characterize crystal phonons and Raman active vibrations in soft matter. We show how to obtain high spectral resolution dispersion data for the resonant part of the third-order optical nonlinearity $\left(\chi^{(3)}\right)$, for both real and imaginary parts, within experiments when the medium is probed with sub-micron spatial resolution. Time-resolved coherent antiStokes Raman scattering (CARS) replicas are detected with femtosecond resolution and can be traced within orders of magnitude of the signal decay. This enables resolution of fine spectral features in dispersion of the resonant third-order nonlinearity that cannot be reliably detected by Raman based spectroscopy techniques. For solid state medium, we focused our study on phonon modes in potassium titanyl phosphate crystal $\left(\mathrm{KTiOPO}_{4}\right.$ or KTP) as the crystal has the most complex vibrational spectra represented by vibrations of different interatomic groups. The material has been shown in a variety of applications as an efficient solid state medium for generation of Raman shifted components within source lasers. The crystal is also a good candidate within applications developing a few femtosecond laser source based on stimulated Raman scattering. Complex phonon lines were analyzed in the KTP 
crystal where we focused on vibrations within titanium octahedron and Ti-O-P intergroup vibrations. For the $\mathrm{TiO}_{6}$ octahedron, we resolved a Raman doublet with a 62 $\mathrm{cm}^{-1}$ frequency difference and slightly different phonon decay times of $495 \mathrm{fs}$ and 515 fs for the two closely spaced vibrations. For phonons representing Ti-O-P intergroup vibrations, we found three components with decay times within the 0.95-1.2 ps range that translate to phonon linewidths of $9.1 \pm 0.4 \mathrm{~cm}^{-1}, 7.5 \pm 0.6 \mathrm{~cm}^{-1}$, and $11.2 \pm 0.5$ $\mathrm{cm}^{-1}$. Comparing Raman active C-C stretching vibrations in fat tissue and oil, we found close similarity between the two. Picosecond dephasing times within the 1.6-2.7 ps range has been detected. Based on the time-domain CARS data, we are able to deduce linewidths as narrow as $4.1 \mathrm{~cm}^{-1}$ in fat tissue which has not been resolved in prior works that used spontaneous scattering and frequency domain coherent Raman spectroscopy methods.

Third-order nonlinear polarization $\left(P^{(3)}\right)$ is the origin of the scattered signal at shifted frequency in coherent Raman spectroscopy. Here, we will consider a case of coherent anti-Stokes Raman scattering (CARS) with three incident fields involved. The nonlinear polarization in the frequency-domain is then expressed in terms of the thirdorder susceptibility $\left(\chi^{(3)}\left(\omega_{\mathrm{as}} ; \omega_{1},-\omega_{2}, \omega_{3}\right)\right)$ and is a nonlinear response of a medium to incident optical fields $\mathrm{E}_{1}, \mathrm{E}_{2}$, and $\mathrm{E}_{3}$ :

$$
P^{(3)}\left(\omega_{a s}\right)=6 \chi^{(3)}\left(\omega_{a s} ; \omega_{1},-\omega_{2}, \omega_{3}\right) E_{1} E_{2}^{*} E_{3},
$$

In the above equation, $E_{1}$ and $E_{2}$ are the complex amplitudes of the driving pulses and $E_{3}$ is the complex amplitude of the time-delayed probe pulse. The nonlinear polarization defined in Equation (1) represents the macroscopic change of polarization connected with the coherent material excitation and probing in the CARS process. If one chooses to 
represent the polarization through the molecular susceptibility tensor $(\alpha)$, the nonlinear polarization expression in frequency-domain can be written as following:

$$
P^{(3)}\left(\omega_{a s}\right)=\frac{1}{2} N \frac{\partial \alpha}{\partial q} Q\left(\omega_{1}-\omega_{2}\right) E_{3},
$$

$\mathrm{N}$ - density of molecules, $\mathrm{Q}$ - coherent amplitude of molecular or lattice vibrations at the driving frequency, $\omega_{\text {as }}$ - anti-Stokes frequency. The interaction between the electromagnetic field and the vibrational mode is described by the molecular susceptibility tensor. These two equivalent definitions of nonlinear polarization in the frequency-domain for the 3-color CARS geometry allows for the definition of the thirdorder susceptibility in terms of the molecular susceptibility tensor and the coherent vibrational amplitude. The macroscopic coherence is the statistical average of molecular displacements $\left(q_{i}\right)$ with Raman frequency $\left(\omega_{R}\right)$ and independent phases $\left(\phi_{i}\right)$. Upon the action of the driving pair of pulses $\left(E_{1}, E_{2}\right)$, the displacements are forced to accept common phase $\left(\phi_{1}-\phi_{2}\right)$. The difference frequency of the pair $\left(\omega_{1}-\omega_{2}\right)$ is tuned to match Raman active vibration $\left(\omega_{R}\right)$ in order to achieve maximum value of $Q$. After the action of the two fields, the coherence decays freely in time as a result of inter- and intraatomic/molecular interactions that lead to changes in the individual phases (pure dephasing) $[25,26]$. Third wave/pulse $E_{3}$, that can be delayed in time with respect to the driving fields, is scattered on the time-dependent coherence $Q(t$,$) producing a signal at$ the anti-Stokes frequency $\left(\omega_{\mathrm{as}}\right)$.

The time-domain coherent amplitude can be represented in terms of an instantaneous response function $G(t)$, that is the characteristic of the ensemble oscillating at Raman frequency $\left(\omega_{\mathrm{R}}=\omega_{1}-\omega_{2}\right)$, coupled to the finite width driving pulses [26]: 


$$
Q(t)=\kappa_{12} \int_{-\infty}^{\infty} G\left(t^{\prime}-t\right) E_{1}\left(t^{\prime}\right) E_{2}\left(t^{\prime}\right) d t^{\prime},
$$

The above equation is a measure of the coherent excitation of the molecules. The stimulated process excites the vibrational system coherently, i.e. the molecules oscillate in unison within the excited volume. The forced common phase then loses uniformity in time due to various dephasing properties. Time-domain CARS signal $\left(S_{a s}\left(t_{d}\right)\right)$ can be expressed as the following:

$$
S_{a s}\left(t_{d}\right)=\kappa \int_{-\infty}^{\infty}|Q(t)|^{2}\left|E_{3}\left(t-t_{d}\right)\right|^{2} d t
$$

The $\kappa$ constant represents combined field coupling coefficients to the molecular/atomic system $\left(\kappa_{12}\right)$ for $E_{1}, E_{2}$ fields and $E_{3}$ field to the coherent amplitude. The time-domain CARS signal, mathematically defined in Equation (4), is a function of the time delay $t_{d}$ corresponding to the time delayed probe pulse. The decay of the time-domain CARS signal (measured experimentally) reveals intrinsic dephasing properties of the coherently excited Raman vibrational mode.

The above equations for the coherent amplitude and CARS signal are Fredholm type-I integral equations and can be solved numerically or using the Fourier transform method [27]. The spectra and autocorrelation functions of the $\mathrm{E}_{1}, \mathrm{E}_{2}$, and $\mathrm{E}_{3}$ pulses are known and can be measured. Provided the condition that $Q(t)$ is a real function, a Fourier transform of the response function $G(t)$ can be obtained. This is true for many types of vibrational systems for which diffusional phase shifting events are minimized or nonexistent. Therefore, fine features in the spectra can be effectively resolved in the vicinity of Raman active vibrations driven by fairly broadband ultrashort pulses. In the case of 
extremely short optical pulses, or when $G(t)$ does not change much on a timescale of the pulsewidths, the integrations are not necessary to calculate the signal $\left(S_{a s}(t)\right)$. Taking into account Equations (1) and (2), a final expression for $\chi^{(3)}$ can be straightforwardly derived:

$$
\chi^{(3)}\left(\omega_{a s} ; \omega_{1},-\omega_{2}, \omega_{3}\right)=\frac{1}{12} N \frac{\partial \alpha}{\partial q} \kappa \int_{0}^{\infty}\left(\frac{S_{a s}(t)}{S_{a s}(0)}\right)^{1 / 2} e^{-i \omega t} d t,
$$

Thus, the experimental CARS transient signal $\left(S_{a s}\left(t_{d}\right)\right)$ can be used as a source function to obtain imaginary and real parts of $\chi^{(3)}$ with the help of Equation (5). We therefore display a quantitative method for deriving the imaginary and real parts of the third-order nonlinearity utilizing the Fourier transform method for solving Equations (3) and (4) and the experimentally measured time-domain CARS signal. A best fit line for the imaginary part of $\chi^{(3)}$, representing the Raman spectrum, then shows evidence of Lorentzian-shaped envelopes corresponding to Raman vibrational modes as well as the corresponding linewidths and modal frequency seperations. The density matrix approach [28] will also bring the same result as the time evolution of the non-diagonal elements and is identical to the one of the coherent amplitude. Coherently driven phonons have been the subject of detailed studies in the past [29].

The experimental design exploits a multicolor femtosecond pulse laser set up that is used here to characterize solid state and biological media. The design also ensures imaging capability with sub-micron spatial resolution. The detection part of the experimental set up is shown in Figure 3.1. As was mentioned above, three-colors are needed to enable time-domain CARS. This is ensured by having time-synchronized optical parametric oscillator (OPO) pulses at two independently tunable wavelengths [30, 31]. The OPOs are driven by the same pump source that is a high-power mode-locked Ti:sapphire oscillator (Coherent Mira HP, 3.5W, 76MHz, $\mathrm{t}_{\mathrm{p}}=110 \mathrm{fs}, 720-960 \mathrm{~nm}$ ). The 
third color is a small fraction that is split from Ti:sapphire oscillator output. The OPO gain material is periodically poled lithium tantalate (PPSLT) with grating period that can be selected for a particular parametric process. Typical power outputs from the OPOs are, depending on wavelength, between 200-375 mW, their pulsewidths are of 115-150 fs, and their pulse bandwidths are in the range of $95-126 \mathrm{~cm}^{-1}$. Continuous tuning and wavelength adjustment at fixed grating period is accomplished by using intracavity Lyott filters and by varying OPO cavity lengths. The three beams are directed to a galvo scanner and imaged onto the entrance pupil of an apochromat objective lens (OBJ) with high numerical aperture $(\mathrm{NA}=1.2)$. A photomultiplier tube (PMT) with high gain and quantum efficiency was used to detect signal photons at wavelengths selected by a combination of bandpass filters and diffraction gratings. The output from the PMT was digitized by a high-speed data acquisition card (DAQ).

Software code (written in Labview) was used for synchronized control of the galvo scanner, acquisition of signal, as well as the formation and display of images in 2D. With the available full angle scan provided by galvo drivers and with the DAQ card, an area of about $180 \times 180 \mu \mathrm{m}^{2}$ could be scanned up to a maximum rate of 4 frames/second. Another software code was used to provide a control of the optical delay line and acquire time-domain CARS signal. Typical point signal average times for sample images and time-domain CARS data were in the range of 1-200 ms, depending on the type of measurement and desired signal-to-noise ratio.

Three-color pulse CARS images at different time-delays can be generated with a spatial resolution of $380 \mathrm{~nm}$. We were able to trace transient non-resonant CARS signals, generated in the forward direction, within higher than five orders of magnitude in solid 
glass material while the average power of the combined beams was kept under $30 \mathrm{~mW}$ $(\sim 0.15 \mathrm{~nJ} /$ pulse per beam $)$ at the sample. The non-resonant time-domain signal in glass (see Figure 3.1(c)) represents instrument function showing our capability to detect decay in coherence with a characteristic dephasing time $\left(T_{2}\right)$ shorter than $150 \mathrm{fs}$. This is shown by comparing the experimental signal (blue ovals) with simulated CARS transients assuming $G\left(t-t^{\prime}\right)=\left\langle q_{i}(t) q_{i}\left(t^{\prime}\right)\right\rangle=h\left(t-t^{\prime}\right) \exp \left(-\left|t-t^{\prime}\right| / T_{2}\right)$, where $h(t)-$ Heavyside function and $T_{2}$ were set to $150 \mathrm{fs}$ (black curve) and $250 \mathrm{fs}$ (red curve). For the measurements presented below, recorded OPO spectra are shown in Figure 3.1 (b). OPO $_{1}$ wavelength was kept fixed at $967 \mathrm{~nm}$ while $\mathrm{OPO}_{2}$ could be tuned to provide access to Raman active vibrations in KTP crystal, oil and fat tissue within $\sim 700-1300 \mathrm{~cm}^{-1}$.

Figure 3.2 (a) shows the time-domain CARS signal obtained in potassium titanyl phosphate $\left(\mathrm{KTiOPO}_{4}\right)$ crystal at a condition where one of the strongest fundamental modes has been driven by a pair of pulses at $1019 \mathrm{~nm}$ and $1098 \mathrm{~nm}$. The crystal is fluxgrown and was angle cut at $\phi=40^{\circ}$ and $\theta=0^{\circ}$. This ensures that the $A_{1}$ and $A_{2}$ symmetry tensor components are involved [32] when $E_{1}, E_{2}$, and $E_{3}$ beam polarizations are set to parallel. The targeted mode is assigned to an asymmetric stretching mode vibration $\left(v_{1}\left(\mathrm{~A}_{1 \mathrm{~g}}\right)\right)$ within $\mathrm{TiO}_{6}$ octahedron [33]. The resonant contribution is dominant, as evidenced by an absence of a resolution limited peak at zero time delay. The experimental CARS signal can be fitted very well by a single exponential decay function for $G(t)$ suggesting that, as expected for high quality crystals, the Raman line is homogenously broadened and corresponds to the loss of vibrational energy via phonon parametric process (rather than pure dephasing) that primarily includes an overtone decay channel. The best fit yields a phonon decay time constant $T_{2}=495 \pm 10$ fs that translates to 
a linewidth $\left(\Delta v=1 /\left(\pi c T_{2}\right)\right)$ of $21.4 \pm 0.5 \mathrm{~cm}^{-1}$. In the case of $967 \mathrm{~nm}$ and $1041 \mathrm{~nm}$ combination for $E_{1}, E_{2}$-pulses, the time domain signal shows clear modulation corresponding to quantum beats of two components separated by $\sim 62 \mathrm{~cm}^{-1}$. The experimental signal shown in Figure 3.2 (b) can be fit well when the two line components $\left(\omega_{\mathrm{R} 1}=706 \mathrm{~cm}^{-1}\right.$ and $\left.\omega_{\mathrm{R} 2}=768 \mathrm{~cm}^{-1}\right)$ are involved. The corresponding response function is then $G(t)=h(t)^{*}\left(A_{1}^{*} \exp \left(-t / T_{21}-i \omega_{R 1}^{*} t\right)+A_{2}^{*} \exp \left(-t / T_{22}-i \omega_{R 2} t\right)\right)$. The best fit is achieved when the amplitude ratio is $9: 5$ and the corresponding $T_{21}, T_{22}$ times of $495 \pm 10$ fs and $515 \pm 10 \mathrm{fs}$. It is obvious that in this case, due to broad bandwidths of $E_{1}, E_{2}-$ pulses $\left(\sim 85 \mathrm{~cm}^{-1}\right)$, the two components can be driven with nearly equal strength opposite to the result shown in Figure 3.2 (a). In other words, the result shown in Figure 3.2 (a) corresponds to phonon mode targeting condition where primarily only one phonon mode centered at (i.e. $\omega_{1}-\omega_{2} \sim 706 \mathrm{~cm}^{-1}$ ) is driven and probed. This results in absence of the beat pattern and decay of the signal that is best fit with the single exponential decay response function presented above. Figures $3.2(\mathrm{c}, \mathrm{d})$ show the corresponding spectra for $\chi^{(3)}$, both for real and imaginary parts, obtained using the formalism described previously that required solutions for the integral Equations (3) and (4). Solid lines are simulated spectra assuming Lorentzian lineshape and line parameters that have been mentioned above. This highlights the asymmetry seen in the spectra obtained using the experimental data. The best fit to the imaginary part is obtained assuming three components with ratios of 26:15:11, linewidths of $21 \mathrm{~cm}^{-1}, 24 \mathrm{~cm}^{-1}, 17 \mathrm{~cm}^{-1}$ and line separations of $65 \mathrm{~cm}^{-1}$ and $16 \mathrm{~cm}^{-1}$, respectively.

Multiple Raman active modes can be driven by $E_{1}, E_{2}$-pulses at the same time, given fairly broad bandwidth pulses. When KTP phonon modes centered at $\sim 830$ 
$\mathrm{cm}^{-1}$ are targeted, a complex nature of Raman active vibrations in the crystal is clearly seen in the detected CARS transients. Figure 3.3(a) shows the time-domain CARS signal obtained for the case of coherently driven vibrations with the center wavelengths for $E_{1}, E_{2}$-pulses adjusted to $967 \mathrm{~nm}$ and $1052 \mathrm{~nm}$, respectively. The signal carries a well pronounced modulation pattern that represents a coherent beat signal due to interference of at least two spectral components. This is governed by the exponential decay trend with a time constant that is clearly longer than the one observed for $v_{1}\left(A_{1 g}\right)$ mode. This is also a substantially longer time than the pulsewidths used in the measurements and the width of the instrument function for the set up. We have therefore analyzed the obtained data for this case with the help of the result expressed by Equation (5). The non-resonant background, due to electronic nonlinearity, has a noticeable presence in the data as can be revealed by Fourier analysis of the CARS transient. The clustered phonon modes at $\sim 830 \mathrm{~cm}^{-1}$ that are driven by the broadband pulses have a weaker contribution, highlighting the fact that the modes have a smaller Raman scattering cross-section compared to the $v_{1}\left(A_{1 g}\right)$ and $v_{2}\left(E_{g}\right)$ modes, as well as narrower linewidths. Raman spectra retrieved from the time-domain signal are represented by the imaginary part of $\chi^{(3)}(\omega)$ and are shown in Figure 3.3 (b). Figure 3.3 (c) shows the corresponding real part of $\chi^{(3)}(\omega)$ in the frequency domain. Three separate components can be clearly seen in the retrieved spectra. The corresponding separations between the components are $18.5 \mathrm{~cm}^{-1}$ and 45.9 $\mathrm{cm}^{-1}$. This is in fairly good agreement with reported spontaneous Raman scattering data that provided the range of $20.4-29.0 \mathrm{~cm}^{-1}$ and $34.6-39.2 \mathrm{~cm}^{-1}$ for the two [34]. Linewidths (FWHM) for the three components are $9.1 \pm 0.4 \mathrm{~cm}^{-1}, 7.5 \pm 0.6 \mathrm{~cm}^{-1}$, and $11.2 \pm 0.5 \mathrm{~cm}^{-1}$, 
respectively. This compares fairly well to ranges of $10.2-12.6 \mathrm{~cm}^{-1}, 9.2-10.8 \mathrm{~cm}^{-1}$, and 14.0-16.4 $\mathrm{cm}^{-1}$, respectively, that have been reported for the spontaneous Raman spectroscopy measurements. A component amplitude ratio of 23:4:15 can also be retrieved and represents the best fit to the imaginary part of the $\chi^{(3)}$ spectra. As was mentioned above, due to a slower decay, phonon linewidths are more than two times narrower when compared to the high frequency modes $\left(v_{l}\left(A_{l g}\right)\right.$ and $\left.v_{2}\left(E_{g}\right)\right)$, as shown in the results presented in Figure 3.2. This is explained by the fact that the $v_{1,2}$ modes have overtone decay channels with a higher phonon density of states for resulting lower energy phonons in parametric decay processes.

Thus, lack of the efficient decay channels for $\sim 830 \mathrm{~cm}^{-1}$ mode leads to a significantly lower damping rate for the driven vibrations. We believe that the mode belongs to Ti-O-P intergroup vibrations opposite to the former case (i.e. $\sim 700 \mathrm{~cm}^{-1}$ ), representing vibrations within the $\mathrm{TiO}_{6}$ atomic group. The presence of the triplet line for the cluster centered at $830 \mathrm{~cm}^{-1}$ can be explained by shifted frequencies for vibrations of different symmetry within the Ti-O-P intergroup.

Raman studies of oil have attracted recently a strong interest in different fields of research. Unlike in crystal lattice, molecules in oil can move freely. Therefore, CARS signal decay is caused by pure dephasing due to collisions and translational motion of molecules. Collisions themselves result in random phase shifts in $q_{i}$. The contribution of the translational motion results in phase changes that are linear in time, due to the Doppler effect in between successive collisions. The dephasing rate due to collisions is uniform and does not depend on velocity distribution. The dephasing due to the Doppler effect (translational motion) is accounted for by averaged phase drift in time due to 
thermal velocity distribution. For a Gaussian distribution, the ensemble averaging results in a real function for $Q(t)$. Namely, $\mathcal{Q}(\mathrm{t})=q_{0} e^{-(\Gamma t-f(t))}$ where $\mathrm{f}(\mathrm{t})=\alpha t^{2}$ for $\mathrm{t} \ll \mathrm{t}_{\mathrm{v}}$ and $f(t)=\beta t$ for $t>t_{v}[35]$. Constants $\alpha$ and $\beta$ are molecular parameters (e.g. mean velocity, diffusion rate coefficient, etc.). Constant $t_{v}$ is velocity correlation time. Figure 3.4 (a) shows time-domain CARS signal for olive oil. The driving fields $\left(\mathrm{E}_{1}\right.$ and $\left.\mathrm{E}_{2}\right)$ have been wavelength tuned to match Raman active mode frequency of $\sim 1072 \mathrm{~cm}^{-1}$. Based on spontaneous Raman data, this is relatively weak C-C stretching vibration. The CARS transient (Fig. 3.4(a)) shows characteristic decay time of about 1.4 ps. The estimated diffusional dephasing time in oil is $\mathrm{T}_{\mathrm{D}}{ }_{\mathrm{D}}=2 / \beta \sim 20 \mathrm{~ms}$. Thus, the obvious conclusion is that the dephasing is entirely due to collisions. Also, the CARS transient exhibits clear modulation (beat pattern), suggesting the presence of a Raman doublet. The corresponding parameters for dephasing times of $2.8 \mathrm{ps}$ and $1.9 \mathrm{ps}$ provided the best fit to the data. By applying a solution provided by Equation (5) to the CARS transient, we can find that the strongest line component on Fig. 3.4(b) fits fairly well into a Lorentzian profile with linewidth $\Delta v_{\mathrm{L} 1}=3.8 \pm 0.1 \mathrm{~cm}^{-1}$, while the second component is noticeably broader $\left(\Delta v_{\mathrm{L} 2}=5.6 \mathrm{~cm}^{-1}\right)$.

The most recent data available in literature for the vibrational mode shows a single peak with a $\sim 7 \mathrm{~cm}^{-1}$ linewidth [36]. The discrepancy is explained by a lack of sensitivity and resolution $\left(\sim 5 \mathrm{~cm}^{-1}\right)$ to provide more precise data.

We have applied our method and experimental capabilities to enable quantitative microscopy where, as an example, we characterized biological tissue. We demonstrate imaging of tissue constituents and then perform dephasing time measurements in targeted areas of the tissue by driving and probing in time characteristic Raman active vibrations. 
Figure 3.5(a) shows a CARS image of mouse tissue (dermis area). The tissue samples were dissected from above the longisimus dorsi muscle of C57BL/6 mice. The image is taken when the delay time between driving $\left(E_{1}, E_{2}\right)$ and probe $\left(E_{3}\right)$ pulses is set to zero. The driven Raman active mode was at a frequency of $\sim 1072 \mathrm{~cm}^{-1}$. The areas filled with dense mouse fat show very strong CARS signals. Collagen fiber bundles can be identified on the CARS image as well. The image contrast in the collagen bundle areas is significantly lower than the one for fat areas. Blood vessel can also be seen on the image with red blood cells (RBC) clearly resolved.

The CARS image shown in Figure 3.5 (b) was obtained at the same conditions except that the probe pulse was delayed by 600 fs. The image contrast between fat and the surrounding areas is drastically improved exceeding one order of magnitude. This is due to the fact that the non-resonant background decays instantaneously in time while the coherence built for the Raman active vibration in fat is still quite appreciable. Figure 3.5 (c) shows CARS signal versus delay time at the same targeting condition for the Raman resonance in fat. The scanning area was reduced $\left(\sim 20 \times 20 \mu \mathrm{m}^{2}\right)$ to match a large piece of fat located at around the center of the image shown in Figure 3.5 (a). Signal fluctuations are fairly high and the signal-to-noise ratio is only about a factor of 5. This is despite the fact that data points in the CARS transient have been effectively averaged across about a 4000 pixel area. The scanning was needed to obtain reproducible time-domain signal. Single point measurement often led to data of poor quality that was the result of tissue damage. This problem will be more closely investigated in our future work. The obtained time-domain CARS signal shows a pronounced beat pattern of at least two spectral components with the corresponding dephasing times of 2.6 and 1.7 ps. The obtained 
transient has been analyzed by generating theoretical curves to fit the experimental data and the results are summarized in the caption. The corresponding dispersion of $\chi^{(3)}$ obtained with the help of Equation (5) is shown on Figure 3.5 (c). It shows a doublet with linewidth parameters that are fairly close (within $15-20 \%$ ) to the ones that were obtained in oil. The molecular composition of oils and lipids in fats are similar, and this should be reflected in Raman spectra. Our data confirms that the fat is responding primarily by its $\mathrm{C}=\mathrm{C}$ double bond fatty acid vibrations that play key roles as signal messengers and lipid mediators in various biological systems.

In conclusion, we have demonstrated femtosecond time-domain coherent antiStokes Raman scattering spectroscopy and microscopy to characterize solid state and biological media. We demonstrated well-resolved Raman active vibrations with a complex line structure that are not routinely resolved by other Raman based spectroscopy techniques. We can obtain distinctly different parameters for complex vibrational lines. Real and imaginary parts of the third-order nonlinear susceptibility near Raman resonances in KTP crystal, oil and animal fat have been obtained with an equivalent spectral resolution of better than $0.1 \mathrm{~cm}^{-1}$. KTP crystal was studied by accessing $\mathrm{TiO}_{6}$ octahedron vibrations. Namely, we have resolved a Raman doublet with $62 \mathrm{~cm}^{-1}$ frequency difference and slightly different phonon decay times of 495 fs and 515 fs for the two closely spaced vibrations. For phonons representing Ti-O-P intergroup vibrations, we found three components with decay times within $0.95-1.2$ ps range that translate to phonon linewidths of $9.1 \pm 0.4 \mathrm{~cm}^{-1}, 7.5 \pm 0.6 \mathrm{~cm}^{-1}$, and $11.2 \pm 0.5 \mathrm{~cm}^{-1}$. Comparing Raman active $\mathrm{C}-\mathrm{C}$ stretching vibrations in fat tissue and oil, we found close similarity between the two. Picosecond dephasing times within 1.6-2.7 ps range has been detected. Based on 
the time-domain CARS data, we are able to deduce linewidths as narrow as $4.1 \mathrm{~cm}^{-1}$ in fat tissue, which has not been resolved in prior works that used spontaneous scattering and frequency domain coherent Raman spectroscopy methods. The experiments have been implemented in a microscopy arrangement, giving strong promise and providing an additional dimension in the comprehensive characterization of condensed matter and biological media. This can lead to a strong impact and important applications in the field of electronic materials that help to identify mechanisms that limit electronic transport parameters. This can also become an effective method in detecting bio-molecular alterations in cells and tissue that is critical for molecular level disease diagnosis.

\section{References}

1. Qu, K. ; Barth, I. ; Fisch, N.J. Plasma wave seed for Raman amplifiers. Phys. Rev. Lett. 2017, 118, 164801-6.

2. Jin, H. ; Liu, F.M. ; Xu, P. ; Xia, J.L. ; Zhong, M.L. ; Yuan, Y. ' ; Zhou, J.W. ; Gong, Y.X. ; Wang, W. ; Zhu, S.N. On-chip Generation and Manipulation of Entangled Photons Based on Reconfigurable Lithium-niobate Waveguide Circuits. Phys. Rev. Lett. 2014, 113, 103601-5.

3. Bhaskar, M.K. ; Sukachev, D.D. ; Sipahigil, A. ; Evans, R.E. ; Burek, M.J. ; Nguyen, C.T. ; Rogers, L.J. ; Siyushev, P. ; Metsch, M.H. ;Park, H. ; Jelezko, F. ; Lonc`ar, M. ; Lukin, M.D. Quantum nonlinear optics with a germanium-vacancy color center in a nanoscale diamond waveguide. Phys. Rev. Lett. 2017, 118, 223603-6.

4. Nguyen, H.A. ; Grange, T. ; Reznychenko, B. ; Yeo, I. ; de Assis, P.L. ; Tumanov, D. ; Fratini, F. ; Malik, N.S. ; Dupuy, E. ; Gregersen, 
N. ; Auffe'ves, A. ; Ge'rard, J.-M. ; Claudon, J. ; Poizat, J.-

P. Giant nonlinear interaction between two optical beams via a quantum dot embedded in a photonic wire. Phys. Rev. B. 2018, 97, 201106-5.

5. Cicchi, R. ; Matthäus, C. ; Meyer, T. ; Lattermann, A. ; Dietzek, B. ; Brehm, B.R. ; Popp, J. ; Pavone, F.S. Characterization of collagen and cholesterol deposition in atherosclerotic arterial tissue using non-linear microscopy. $J$. Biophotonics 2014, 7, 135-43.

6. Denk, W.; Strickler, J.H.; Webb, W.W. Two-photon laser scanning microscopy. Science 1990, 248, 73-76.

7. Campagnola, P.J.; Millard, A.C.; Terasaki, M.; Hoppe, P.E.; Malone, C.J.; Mohler, W.A. Three-dimensional high-resolution second-harmonic generation imaging of endogenous structural proteins in biological tissues. Biophys. J. 2002, $82,493-508$.

8. Débarre, D.; Suppato, W.; Pena, A.M.; Fabre, A.; Tordjmann, T.; Combettes, L.; Schanne-Klein, M.-C.; Beaurepaire, E. Imaging lipid bodies in cells and tissues using third-harmonic generation microscopy. Nat. Meth. 2006, 3, 47-53.

9. Evans, C.L.; Potma, E.O.; Puoris'haag, M.; Coté, D.; Lin, C.P.; Xie, X.S. Chemical imaging of tissue in vivo with video-rate coherent anti-Stokes Raman scattering microscopy. Proc. Natl. Acad. Sci. USA 2005, 102, 16807-16812.

10. Freudiger, C.W.; Min, W.; Saar, B.G.; Lu, S.; Holtom, G.R.; He, C.; Tsai, J.C.; Kang, J.X.; Xie, X.S. Label-free biomedical imaging with high sensitivity by stimulated Raman scattering microscopy. Science 2008, 322, 1857. 
11. Widulle, F.; Ruf, T.; Schonherr, E.; Cardona, M. Investigation of the anomalous Raman line shape of the TO phonon in GaP. Phys. Status Solidi B 1999, 215, $131-6$.

12. Joshi, J. ; Stone, I.R.; Beams, R.; Krylyuk, S.; Kalish, I.; Davydov, A.V.; Vora, P.M. Phonon anharmonicity in bulk $\mathrm{T}_{\mathrm{d}}-\mathrm{MoTe}_{2}$. Appl. Phys. Lett. 2016,109, 031903-5.

13. Kaminskii, A.A.; Lux, O.; Rhee, H.; Eichler, H.J.; Yoneda, H.; Shirakawa, A.; Ueda, K.; Zhao, B.; Chen, J.; Dong, J.;Zhang, J. Crystal-host $\mathrm{Gd}_{0.5} \mathrm{Lu}_{0.5} \mathrm{VO}_{4}$ for $\mathrm{Ln}^{3+}$-lasants: a new high-gain many-phonon $\chi^{(3)}$-active tetragonal vanadate-SRS spectroscopy and nonlinear-laser effects. Appl. Phys. B 2012,109, 649-58.

14. Kaminskii, A.A. ; Lux, O.; Rhee, H.; Kaltenbach, A.; Eichler, H.J.; Zhang, J.; Tang, D.Y.; Yu, H.; Zhang, H.; Wang, J.;Yoneda, H.; Ueda, K.; Shirakawa, A. Low-temperature stimulated Raman scattering spectroscopy of tetragonal $\mathrm{GdVO}_{4}$ single crystals. Phys. Status Solidi B 2014, 251, 1045-62.

15. Liu, W.; Wang, H.; Du, J; Jing, C. Raman microspectroscopy of nucleus and cytoplasm for human colon cancer diagnosis. Biosens. Bioelectron 2017, 97, 70.

16. Eberhardt, K.; Beleites, C.; Marthandan, S.; Matth€aus, C.; Diekmann, S.; Popp, J. Raman and Infrared Spectroscopy Distinguishing Replicative Senescent from Proliferating Primary Human Fibroblast Cells by Detecting Spectral Differences Mainly Due to Biomolecular Alterations. Anal. Chem. 2017, 89, 2937.

17. Thomas, G.J. Raman spectroscopy of protein and nucleic acid assemblies. Annu. Rev. Biophys. Biomol. Struct. 1999, 28, 1. 
18. Korifi, R.; Le Dreau, Y.; Molinet, J.; Artaud, J.; Dupuy, N. Composition and authentication of virgin olive oil from French PDO regions by chemometric treatment of Raman spectra. J. Raman Spectrosc. 2011, 42, 1540.

19. Potma, E.O.; Evans, C.L.; Xie, X.S. Heterodyne coherent anti-Stokes Raman scattering (CARS) imaging. Optics Letters 2006, 31(2), 241-243.

20. Saar, B.G.; Freudiger, C.W.; Reichman, J.; Stanley, C.M.; Holtom, G. R.; Xie, X.S. Video-Rate Molecular Imaging in Vivo with Stimulated Raman Scattering. Science 2010, 330(6009), 1368-1370.

21. Racu, A.-M.; Menzel, D.; Schoenes, J.; Doll, K. Crystallographic disorder and electron-phonon coupling in $\mathrm{Fe}_{1-\mathrm{x}} \mathrm{Co}_{\mathrm{x}} \mathrm{Si}$ single crystals: Raman spectroscopy study. Phys. Rev. B. 2007, 76, 115103-7.

22. Giarola, M. ; Sanson, A.; Rahman, A.; Mariotto, G. ; Bettinelli, M. ; Speghini, A. ; Cazzanelli, E. Vibrational dynamics of $\mathrm{YPO}_{4}$ and $\mathrm{ScPO}_{4}$ single crystals: An integrated study by polarized Raman spectroscopy and first-principles calculations. Phys. Rev. B. 2011, 83, 224302-8.

23. Pavillon, N. ; Hobro, A.J.; Smith, N.I. Cell optical density and molecular composition revealed by simultaneous multimodal label-free imaging. Biophys. J. 2013, 105, 1123-32.

24. Corsetti, S. ; Rabl, T.; McGloin, D.; Nabi, G. Raman spectroscopy for accurately characterizing biomolecular changes in androgen-independent prostate cancer cells. J. Biophotonics 2018, 11, 1-8.

25. Giordmaine, J. A.; Kaiser, W. Light scattering by coherently driven lattice vibrations. Physical Review 1966, 144(2), 676. 
26. Laubereau, A.; Kaiser, W. Vibrational dynamics of liquids and solids investigated by picosecond light pulses. Reviews of Modern Physics 1978, 50(3), 607.

27. Jerry, A. Introduction to Integral Equations With Applications, $2^{\text {nd }}$ ed.; John Wiley \& Sons, Inc., 1999.

28. Malinovskaya, S.A. Prevention of decoherence by two femtosecond chirped pulse trains. Optics letters 2008, 33(19), 2245-2247.

29. Kutt, W.A.; Albrecht, W.; Kurz, H. Generation of Coherent Phonons in Condensed Media. IEEE Journal of Quantum Electronics 1992, 28(10), 24342444.

30. Rowley, J.; Yang, S.; Ganikhanov, F. Power and tuning characteristics of a broadly tunable femtosecond optical parametric oscillator based on periodicallypoled stoichiometric lithium tantalate. JOSA B 2011, 28, 1026-1036.

31. Yang, S.; Adhikari, S.; Dobbala, M.; Adusumilli, S.; Rowley, J.; Zhang, L.; Marrs, G.; Wysolmerski, R.; Spirou, G.; Ganikhanov, F. Multi-color ultrafast laser platform for nonlinear optical imaging based on independently tunable optical parametric oscillators. Appl. Phys. B: Lasers and Optics 2013, 111, 617625 .

32. Kugel, G. E.; Brehat, F.; Wyncke, B.; Fontana, M. D.; Marnier, G.; CarabatosNedelec, C.; Mangin, J. The vibrational spectrum of a KTiOPO4 single crystal studied by Raman and infrared reflectivity spectroscopy. Journal of Physics $C$ : Solid State Physics 1988, 21(32), 5565.

33. Vivekanandan, K.; Selvasekarapandian, S.; Kolandaivel, P.; Sebastian, M. T.; Suma, S. Raman and FT-IR spectroscopic characterisation of flux grown 
KTiOPO4 and KRbTiOPO4 non-linear optical crystals. Materials chemistry and physics 1997, 49(3), 204-210.

34. Watson, G. H. Polarized Raman spectra of ktioaso4 and isomorphic nonlinearoptical crystals. J. Raman Spectrosc. 1991, 22(11), 705-713.

35. D'yakov, Y. E. Dephasing in steady-state and time-varying spectroscopy. JETP Lett. 1983, 37, 15.

36. El-Abassy, R.M.; Donfack, P.; Materny, A. Visible Raman spectroscopy for the discrimination of olive oils from different vegetable oils and the detection of adulteration. J. Raman Spectrosc 2009, 40, 1284. 


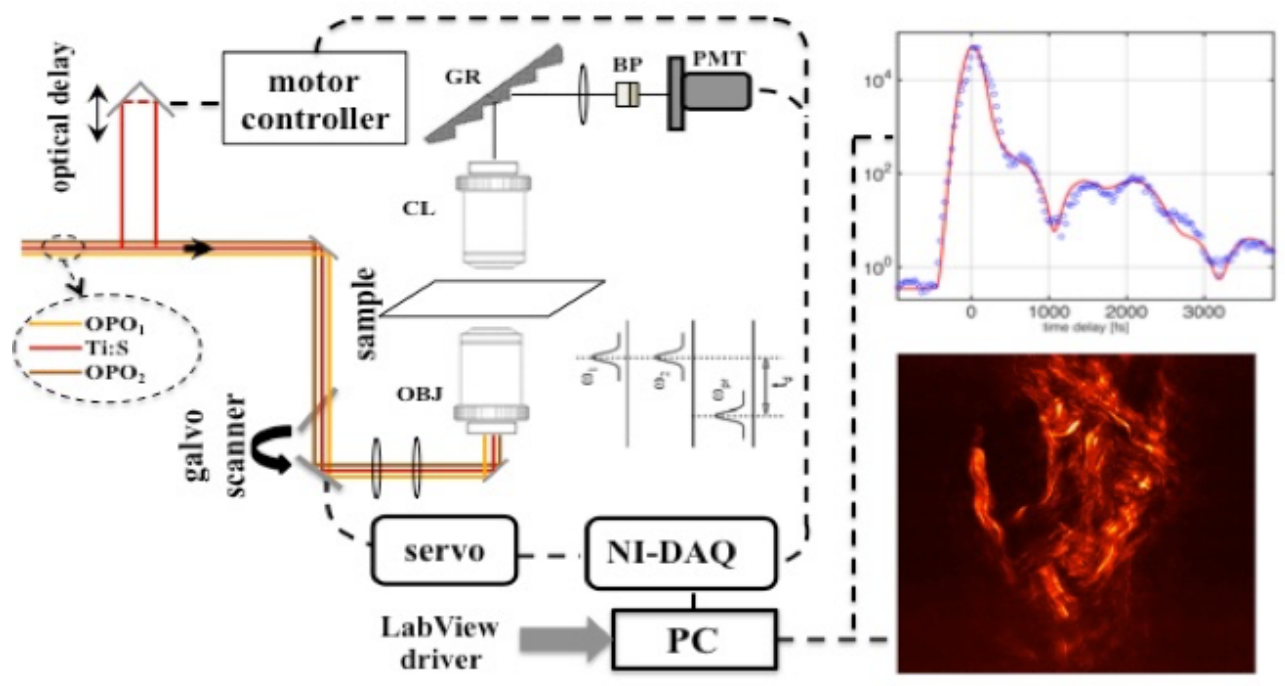

(a)

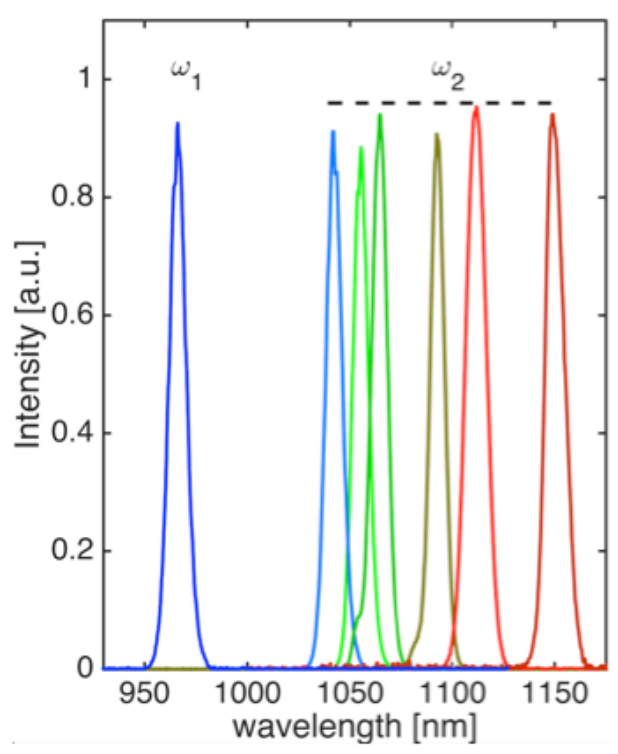

(b)

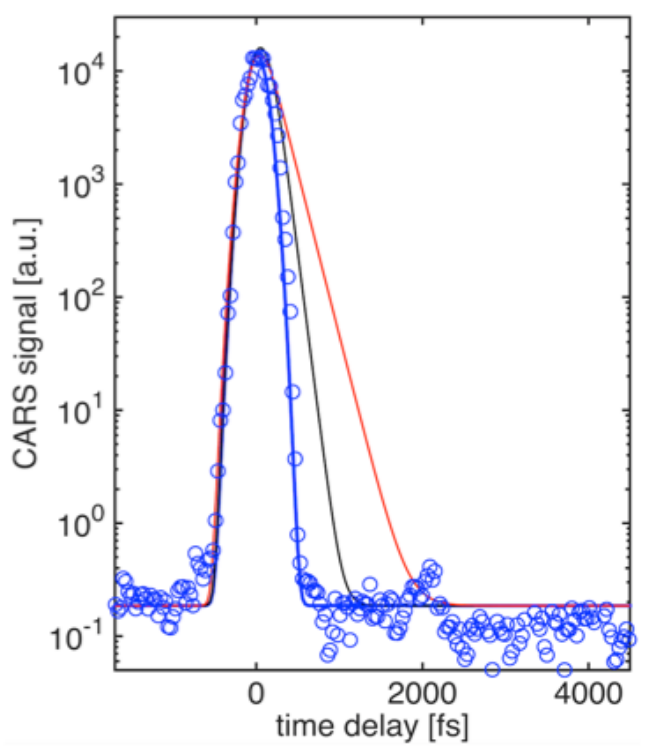

(c)

Figure 3.1: (a) Experimental set up. The three-color pulses at $\omega_{1}, \omega_{2}, \omega_{3}$ are provided from lab built tunable femtosecond optical parametric oscillator $\left(\mathrm{OPO}_{1,2}\right)$ sources and fixed wavelength Ti:sapphire oscillator (Coherent Mira) respectively. GR- diffraction grating 1200 1/mm, BP-bandpass filter, CL-collimating lens objective. Galvo-scanner is from Cambridge Technology, Inc. (model \#6210), photomultiplier tube (PMT) is from Hamamatsu (model \#H9305), data acquisition card (DAQ) is from National Instruments 
(model 6201); (b) $\mathrm{OPO}_{1,2}$ spectra for nearly transform limited $\mathrm{E}_{1,2}$ pulses used for CARS experiments; (c) Time-domain CARS signal (open circles) obtained from microscope glass when the OPO wavelengths were set to $967 \mathrm{~nm}$ and $1152 \mathrm{~nm}$. The transient signal represents instrument function that allows to measure dephasing times $\left(T_{2}\right)$ shorter than 150 fs. Solid curves are simulated CARS signals that make use Equations (3) and (4) and assuming single exponential decay for $G(t)$ with $150 \mathrm{fs}$ (black) and $250 \mathrm{fs}$ (red) dephasing times.

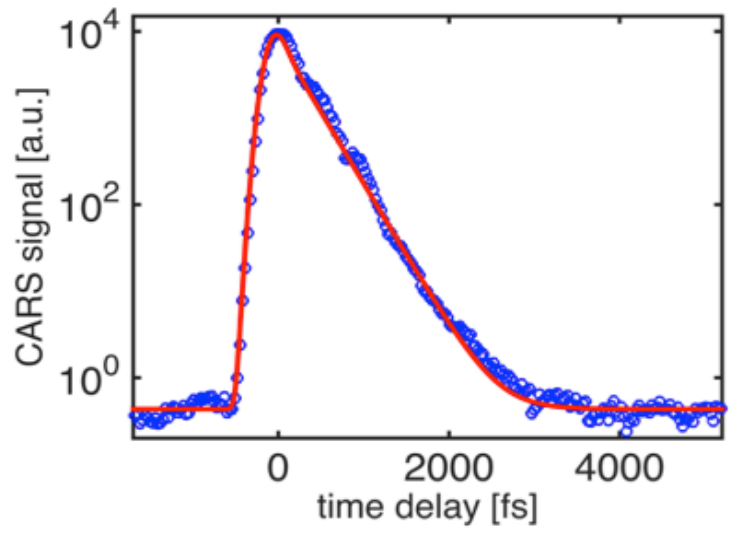

(a)

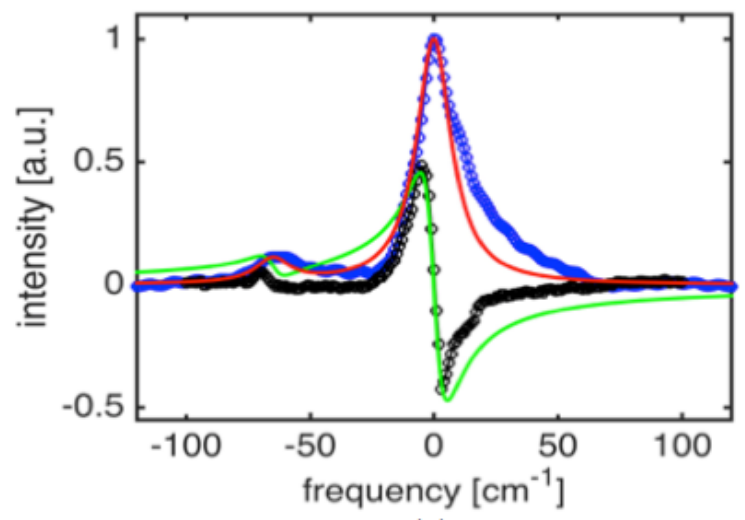

(c)

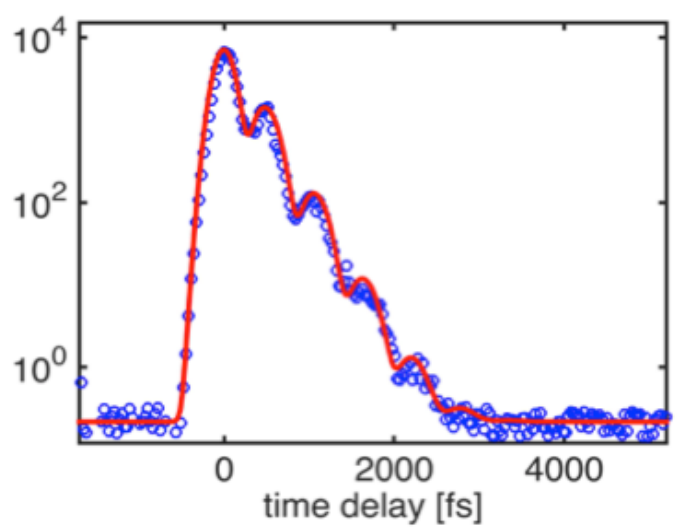

(b)

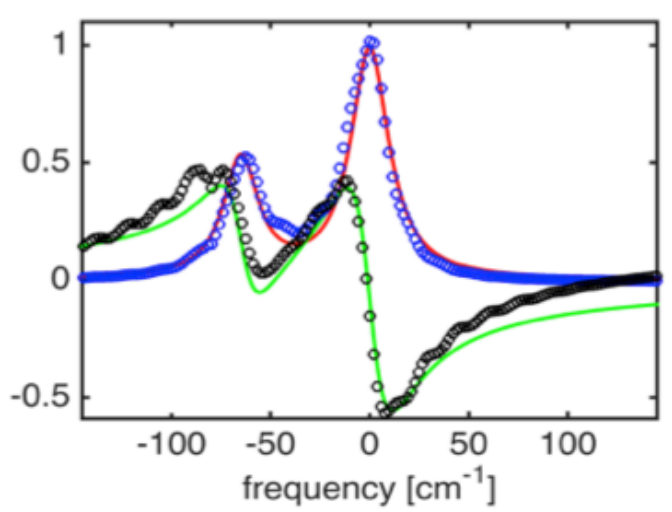

(d)

Figure 3.2: (a) Time-domain CARS signal (open circles) obtained from KTP crystal when the OPO wavelengths were set to $1019 \mathrm{~nm}$ and $1098 \mathrm{~nm}\left(\sim 706 \mathrm{~cm}^{-1}\right)$. Red solid line represents theoretical fit that assumes single exponential decay for $G(t)$ with the 
dephasing time of $495 \mathrm{fs}$; (b) Time-domain CARS signal (open circles) obtained from KTP crystal when the OPO wavelengths were set to $967 \mathrm{~nm}$ and $1041 \mathrm{~nm}$. Solid line represents theoretical fit that assumes Raman doublet with $62 \mathrm{~cm}^{-1}$ frequency difference and decaying with the dephasing times of 495 fs and $515 \mathrm{fs}$; (c, d) Corresponding spectra of the resonant third order nonlinearity obtained with the help of the formalism presented in the text. Real part of $\chi^{(3)}$ is shown in black open circles while the imaginary part is shown in blue circles. Solid lines show results of simulations for both parts of the nonlinearity assuming Lorentzian profile for the two components with parameters outlined in the text. 

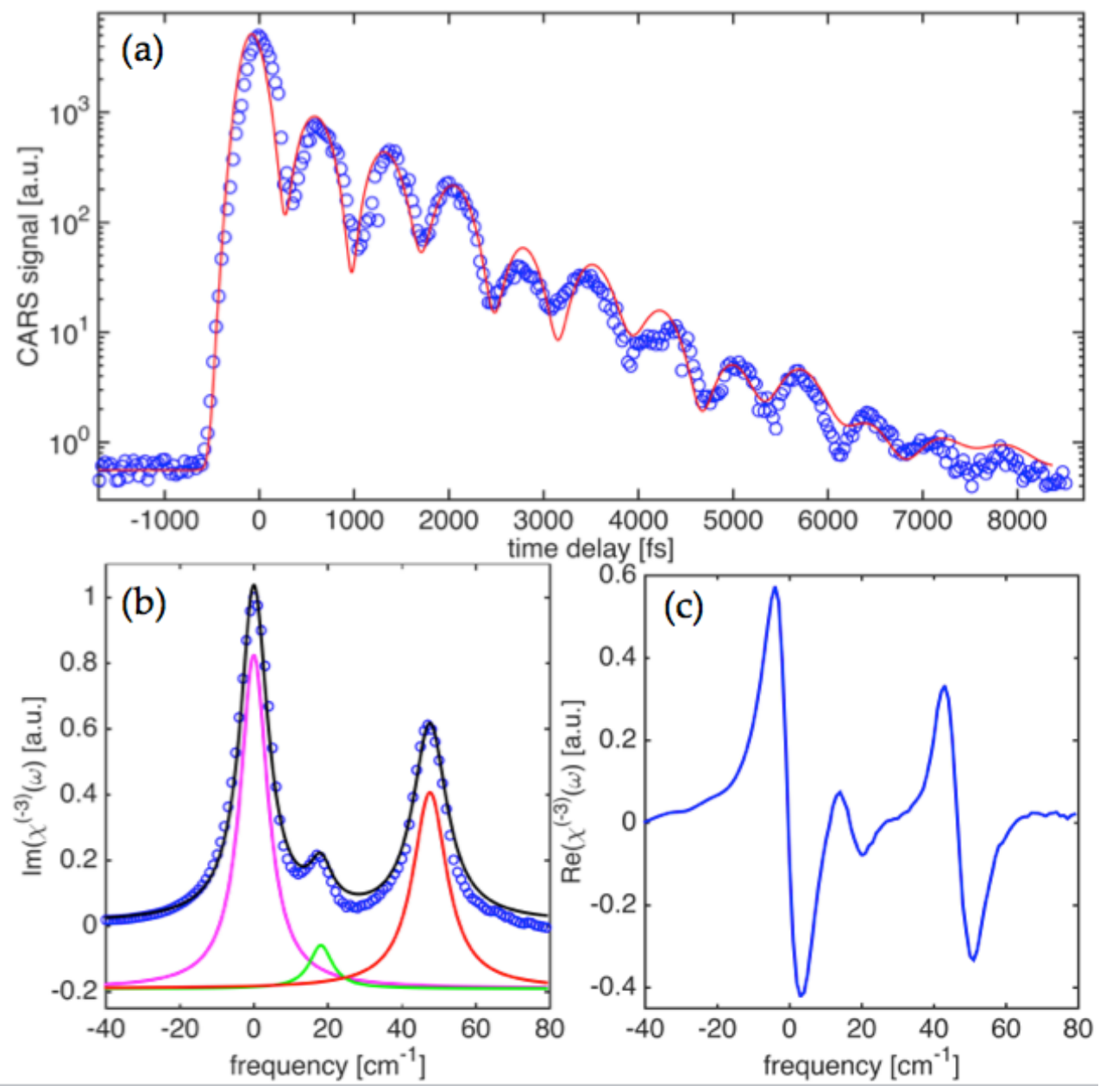

Figure 3.3: (a) CARS transients (open circles) obtained from KTP crystal when the OPO wavelengths were set to $967 \mathrm{~nm}$ and $1052 \mathrm{~nm}$ ( $\sim 830 \mathrm{~cm}^{-1}$ vibration). Solid line represents theoretical fit that assumes three spectral components and is obtained using software algorithm that varies multiple parameters for each component (amplitude, spectral position, dephasing time) variation that assumes Raman doublet with $62 \mathrm{~cm}^{-1}$ frequency difference and decaying with the dephasing times of $950 \mathrm{fs}$ and $1160 \mathrm{fs}$; (b) Corresponding spectrum of the resonant third order nonlinearity $\left(\chi^{(3)}(\omega)\right)$ obtained using experimental CARS transient and applying Equation (5). Imaginary part of $\chi^{(3)}$ is shown in blue open circles. Solid black line is the best fit to the spectrum assuming three 
components shown in separate colors and with parameters provided in the text; (c) Real part of $\chi^{(3)}(\omega)$.

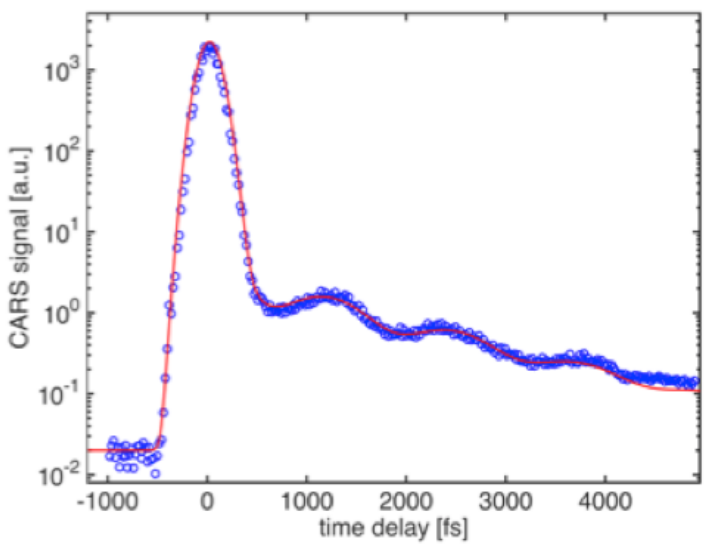

(a)

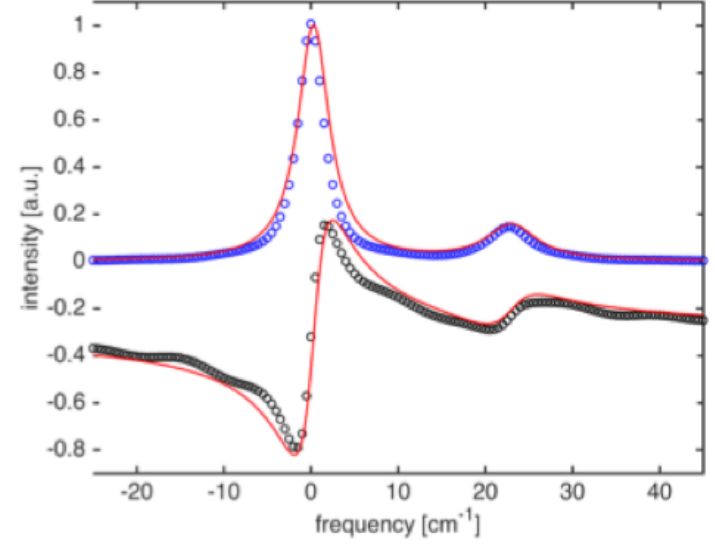

(b)

Figure 3.4: (a) CARS transients (open circles) measured in olive oil while vibrations at $\approx 1072 \mathrm{~cm}^{-1}$ were driven and probed. The best fit to the data is given by solid red line for which Equations (3)-(4) have been employed. Corresponding line component parameters are listed in the text; (b) Real (black circles) and imaginary (blue circles) parts of $\chi^{(3)}(\omega)$ obtained using experimental CARS transient and Equation (5). 


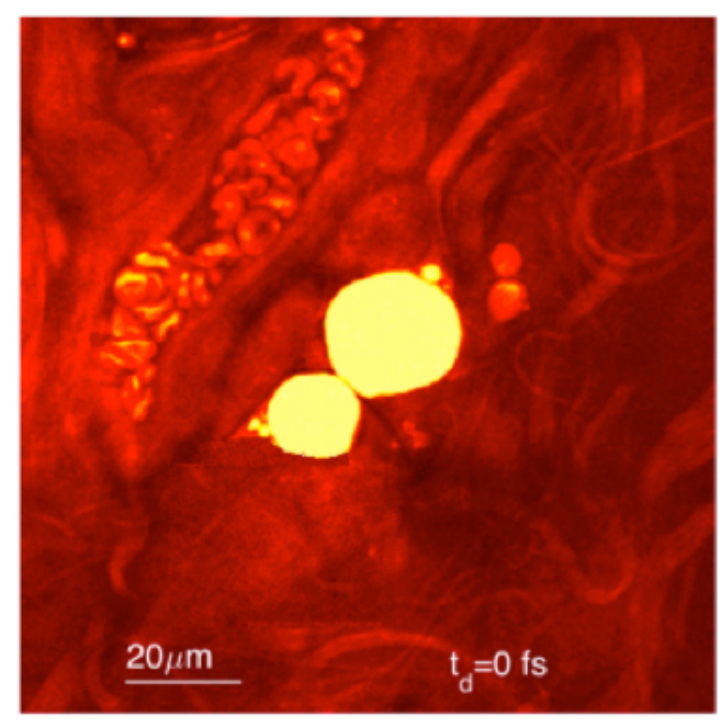

(a)

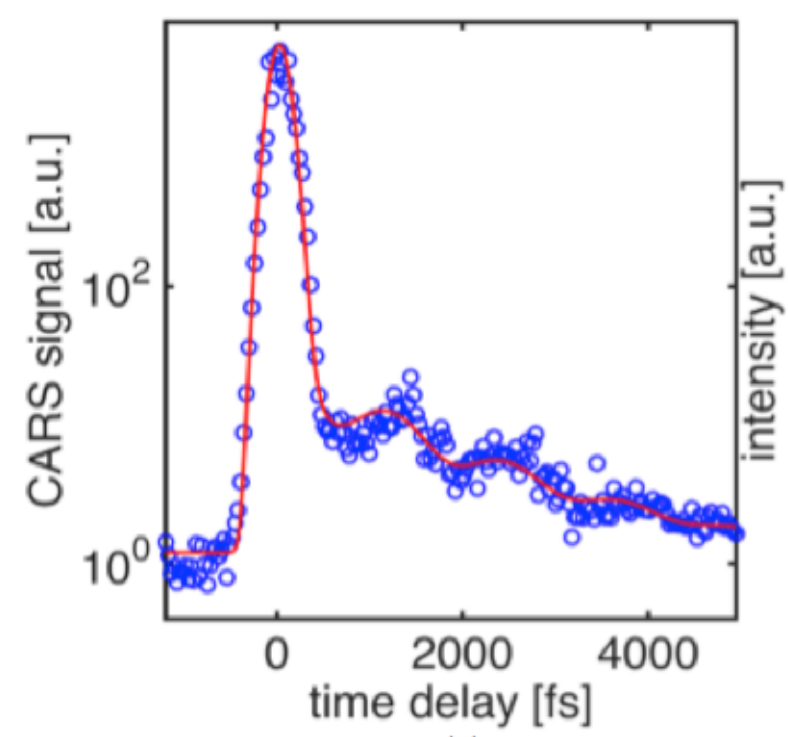

(c)

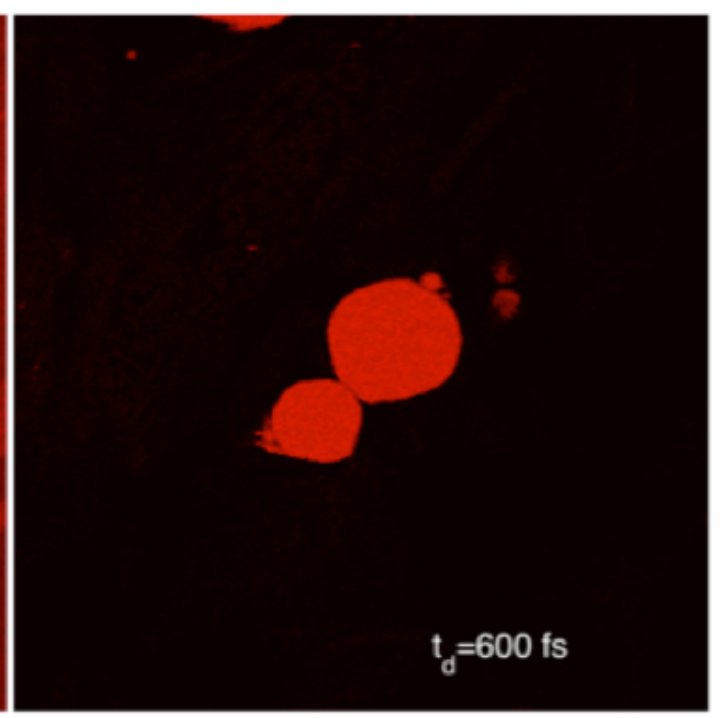

(b)

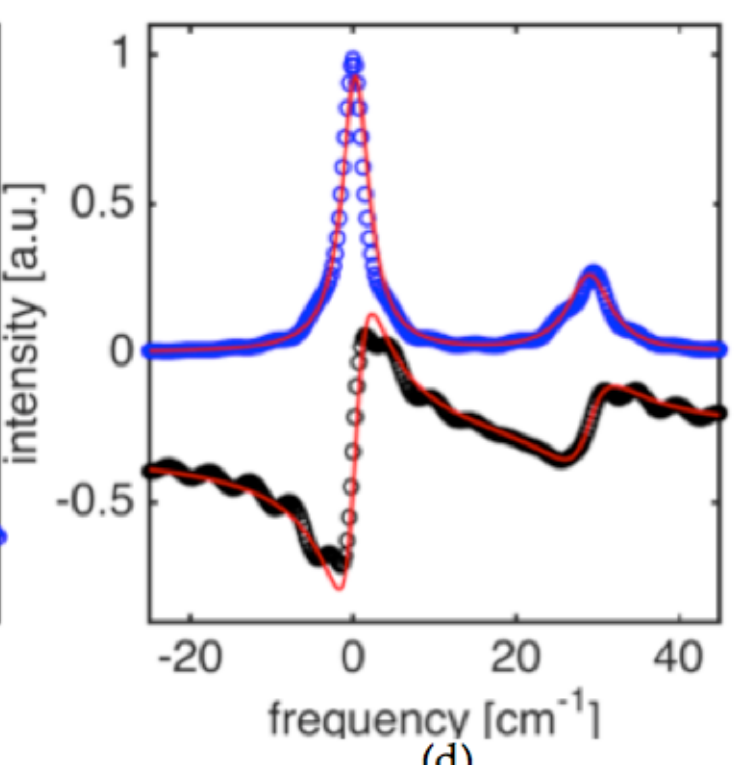

(d)

Figure 3.5: (a) CARS image obtained from the mouse dermis area at zero time delay between the driving $E_{1}, E_{2}$ pulsed fields and the probe field $E_{3}$. Vibration at $1072 \mathrm{~cm}^{-1}$ has been targeted; (b) CARS image the same area when the probe pulse was delayed by $600 \mathrm{fs}$; (c) Time-domain CARS signal obtained from the $\sim 20 \mu \mathrm{m}$ diameter fat area located at the center of the mouse tissue for the image shown in Fig. 5(a). Red line represents the best fit to the data obtained by using Equations (3)-(4) and varying the 
corresponding line parameters (see text); (d) Real (black circles) and imaginary (blue circles) parts of $\chi^{(3)}(\omega)$ obtained using the experimental CARS transient shown in part (c) and Equation (5). Red line is theoretical fit that makes use two-component Lorentzian function ( $\Delta v_{1,2}=4.1,6.2 \mathrm{~cm}-1,28 \mathrm{~cm}^{-1}$ separation between components, $\left.\mathrm{A}_{1} / \mathrm{A}_{2}=13: 4\right)$. 


\section{CHAPTER 4}

Published in American Institute of Physics (AIP) Advances, October 2017

Dispersion of the resonant second order nonlinearity in 2D semiconductors probed by femtosecond continuum pulses

Mohammed Mokim, Adam Card, Bindeshwar Sah, and Feruz Ganikhanov

Department of Physics, University of Rhode Island, 2 Lippitt Road,

Kingston, RI, 02881.

Corresponding Author: $\quad$ Feruz Ganikhanov, Ph. D.

Department of Physics

University of Rhode Island

East Hall, 2 Lippitt Road

Kingston, RI, 02881, USA

Phone: (401) 874-2633

Email address: fganikhanov@uri.edu 
Two-dimensional materials have attracted very strong interest due to their promise in practical applications. For example, monolayer $\mathrm{MoS}_{2}$ has been shown recently as an efficient material for low-power field effect transistors [1]. Strongly enhanced second order optical nonlinearity $\chi^{(2)}$ in monolayers of transition metal dichalcogenides (TMDC) is also predicted due to the lack of an inversion center in their crystalline structure. The reported absolute values of $\chi^{(2)}$ vary though by orders of magnitude $5-10^{5}$ $\mathrm{pm} / \mathrm{V}[2-4]$. Very few studies though address the spectral response of $\chi^{(2)}$. This type of data can provide new information along with the results of traditional optical methods for condensed matter characterization. Wang et al. [5] performed experiments on obtaining $\chi^{(2)}$ dispersion in $\mathrm{WSe}_{2}$ at low temperatures. Multiple peaks in $\chi^{(2)}$ dispersion within SHG photon energy range of 1.7-2.4 eV were clearly attributed to excitons with $n=1-3$. Large variations in the SHG signal have made this point-by-point measurement insensitive to smaller features in the SHG spectra. In addition, the incident photon energy range was limited so that the near bandgap nonlinear optical response of the material could not be obtained.

In this letter, we report on a microspectroscopy method applied to characterize monolayer $\mathrm{WSe}_{2}$ within a photon energy range of $2.4-3.2 \mathrm{eV}$. We detect single-shot second harmonic (SH) spectra from monolayer $\mathrm{WSe}_{2}$ material with better than $0.3 \mathrm{~nm}$ resolution $(\sim 3 \mathrm{meV})$ and low noise $(5-6 \% \mathrm{rms})$ using broadband femtosecond continuum pulses. Fine sub-structure features that can be detected within the main peak of $\chi^{(2)}$ indicate the impact of near bandgap exciton transitions. We retrieve, with a fairly good precision, the fundamental bandgap and exciton binding energy. The absolute values for 
$\chi^{(2)}$ are also provided. We believe that our experimental results will aide in developing refined theoretical models for 2D materials.

The experimental idea is presented in Fig. 4.1(a). Femtosecond continuum pulses with a smooth spectral envelope centered in the near-IR are used to generate SHG signal within an atomically thin semiconductor sample. Spectrum of the second harmonic signal that carries spectral signatures of the sample is then detected with fine $(\sim 3 \mathrm{meV})$ spectral resolution. The laser part of the experimental arrangement, shown in Fig. 4.1(b), generates spectrally smooth shape for the ultra-broad continuum in the near infrared (780-1050 nm) with typical power density of $\sim 50 \mu \mathrm{W} / \mathrm{nm}$. Chromatic dispersion compensated optics were used to deliver the fundamental beam to the sample within less than $400 \mathrm{~nm}$ spotsize. The incident beam can be angle scanned using a galvo-mirror scanner to provide $200 \times 200 \mu \mathrm{m}^{2}$ imaging area. SH signal from the sample was collected through the same objective lens in the backward direction. The SHG signal spectra were resolved using a monochromator (Horiba, Inc. model: iHR320) and a cooled CCD detector (Syncerity-UV/Vis, Horiba Inc.). Single layer $\mathrm{WSe}_{2}$ flakes were prepared by

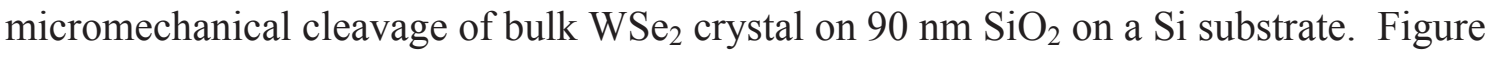
4.2(a) shows SHG image of the flake. The flake was also characterized in a separate photoluminescence measurement. The latter reveals a narrow $(\sim 45 \mathrm{meV})$ peak at $746 \mathrm{~nm}$ $(\sim 1.662 \mathrm{eV})$ that is shown in Fig. 4.2(b) and corresponds to the first exciton line characteristic for single layer. We have checked the SHG signal dependency versus incident power of the fundamental beam to reveal the quadratic increase shown in Fig. 4.2(c). The SHG image shown in Fig. 4.2(a) displays high contrast and an absence on any appreciable signal from interfaces other than the one created by the flake. Fairly large 
SHG signal variations (up to $30 \%$ ) are observed even within the unripped parts of the flake (Fig. 4.2(d)). We believe that this is due to local field variations affecting $\chi^{(2)}$ at the flake/ $\mathrm{SiO}_{2}$ interface. The $\mathrm{SHG}$ spectrum is shown in Fig. 4.3(a) by the blue curve. We have also performed, for comparison purposes, point-by-point wavelength tuning SHG measurements with wavelength tunable Ti:sapphire oscillator (filled circles data in Fig. 4.3(a)). The SHG signal fluctuations are significantly higher $(\sigma= \pm 54 \%)$ for this case. We believe that much higher SHG signal variations versus wavelength observed in the pointby-point measurements are due to couple of additional sources of the variation. Namely, changes in the fundamental beam parameters like pulsewidth and spatial mode while the wavelength is tuned. The lowered precision for the SHG spectra were also observed in the referred point-by-point measurements [5] as authors pointed to the uncertainties in the puslewidths while the wavelength was tuned as being the main reason.

The observed increase in the resonant nonlinearity $\chi_{2 D}^{(2)}$ matches well with splitoff band transitions (i.e. B-exciton) if one considers bandstructure parameters for single layer of $\mathrm{WSe}_{2}$ at K-point such as bandgap $\left(E_{g}\right)$ and split-off energy $\left(\Delta_{S O}\right)[5,6]$. A similar effect has been observed in $\mathrm{MoS}_{2}$ involving a different energy valley that is at $\Gamma$ point [3].

We have obtained $\chi_{2 D}^{(2)}$ spectra using two approaches. The first one exploits the relationship between the fundamental and SH powers. In the other one, we used a comparative approach when a material with known second order nonlinearity is used. We have chosen thin KTP crystal. The crystal is well characterized from many aspects. The second order effective nonlinearity $\left(d_{e f f}\right)$ is between $1.72-2.01 \mathrm{pm} / \mathrm{V}[7]$ for the incoming beam polarization and crystal orientation that we used. By normalizing our $\mathrm{WSe}_{2} \mathrm{SH}$ data 
to the one obtained from the crystal $\left(P_{2}^{B}\right)$, we obtain a ratio $\left(\rho=P_{2}^{2 D} / P_{2}^{B}\right)$ that provides dispersion of the absolute value of $\chi_{2 D}^{(2)}$ and is free from measurement artifacts (e.g., $T_{2 \omega}$, etc.). The result is displayed in Fig. 4.3(b) by blue curve for a comparison. Some difference can be seen at lower photon energies. We note that SHG and fundamental signal have been detected by different spectral devices in the first method. The second method is free from the artifact.

Bloembergen et al [8] were first to consider SHG from thin layers and interfaces. SHG power has been derived by Merano [9] by considering a real experimental situation of a sheet of 2D material on the top of the layered substrate. Following the approach developed in $[9,10]$ and making use of the results by Boyd et al. [11] SHG pulse peak power from 2D material $\left(P_{2}^{2 D}\right)$ and bulk crystal $\left(P_{2}^{B}\right)$ can be expressed as shown in Supplement [12]. Based on that, the absolute value of $\chi_{2 D}^{(2)}$ expressed in terms the crystal's effective nonlinearity $d_{\text {eff }}$, refractive index $\left(n_{b}\right)$, numerical aperture of the objective lens $(\Theta)$ and the measured ratio $(\rho)$ is:

$$
\chi_{2 D}^{(2)}=\frac{16 \lambda_{1} \sqrt{\rho}}{\left|\left(1+r_{\omega}\right)^{2}\left(1+r_{2 \omega}\right)\right|\left(n_{b}+1\right)^{3} \sqrt{n_{b}} \Theta^{2}}\left|d_{e f f}\right|
$$

The above equation is an estimate of the second-order nonlinearity for the atomically thin crystal in terms of the normalization of the two-dimensional second harmonic data compared to the previous data from a reference crystal. Using this approximation eliminates the artifact corresponding to the second-order nonlinearity measurement obtained by the power relationship method.

It is important to note that the two-layered substrate can lead to the enhancement or attenuation of the SHG intensity due to interference effects that depends on the 
thickness of the $\mathrm{SiO}_{2}$ layer. This is taken into account in the factor containing complex field reflectivity $\left(r_{\omega}, r_{2 \omega}\right)$. With the help of Equation (1), we can provide absolute scaling for the result shown in Fig. 4.3(b) (left scale). For KTP material the refractive index $\left(n_{b}\right)$ changes by only few percent within the photon energy range that we have. The median peak value of $\chi_{2 D}^{(2)}$ for $\mathrm{WSe}_{2}$ monolayer at the $\mathrm{SH}$ photon energy of $2.76 \mathrm{eV}$ can be estimated at $7.3 \times 10^{-19} \mathrm{pm}^{2} / \mathrm{V}$. We provide $\pm 15 \%$ range owing to several factors such as the range for $d_{\text {eff }}$ itself and taking into account signal variations across the flake that were discussed earlier. The value is on the same order of magnitude with the one quoted for the measured $\mathrm{MoS}_{2}$ sheet nonlinearity in [13] and factor of 2.7 smaller than the one provided by Merano [9] for $\mathrm{MoS}_{2}$. If we straightforwardly use solutions provided in [8] and assume that the sample still has bulk refractive index [14] and nonlinearity $\chi_{2 D}^{(2)}$ (in units of $\mathrm{m} / \mathrm{V}$ ) the expression for estimate is:

$$
\left|\chi_{2 D}^{(2)}\right|=\sqrt{\rho} \frac{8 \pi \sqrt{f\left(n^{\prime}\right)}}{\sqrt{n}(n+1)^{3} \Theta^{2}|\zeta|}\left|\chi_{B}^{(2)}\right|
$$

The above equation is an estimate for the second-order nonlinearity when the $2 \mathrm{D}$ comparison layer is treated as a bulk-like system. In this approach, one must consider the SHG process in bulk media under tight focusing conditions as well as the treatment of the SHG problem in the single-layer sample.

In the formula above, $f\left(n^{\prime}\right)$ is a factor containing refractive indices of bulk $\mathrm{WSe}_{2}$ and is coming from solutions for the $\mathrm{SH}$ field [17], $\zeta$-factor accounts for the interference effects. Additional details are provided in Supplement [12]. This approach may cause controversy and disagreement. We, however, we would like to provide an estimate just for sake of comparing the material's nonlinearity with the one of other bulk non- 
centrosymmetric semiconductors. The estimated $\chi_{2 D}^{(2)}$ value is in the $932-1233 \mathrm{pm} / \mathrm{V}$ range for peak SH photon energy while the off-peak value is about $440 \mathrm{pm} / \mathrm{V}$. The values are comparable with the ones for well known non-centrosymmetric semiconductors (GaAs, CdTe, ZnSe [15]) that are used in parametric devices and frequency converters in the infrared [16]. Comprehensive theoretical treatment and modeling of the second order nonlinearity is based on rigorous approaches outlined in $[17,18]$. Here we will use an approach based on parabolic bands approximation, accounting for Coulomb effects via exciton continuum states above bandgap in order to estimate dispersion of the absolute value of $\chi^{(2)}$ due to interband transitions first. The expression for $\chi^{(2)}$ along the electric field and induced dipole moment in $x$-direction can be represented by [18]:

$$
\begin{gathered}
\chi_{X X X}^{(2)}(2 \omega)=-\frac{i e^{3}}{2 m^{3} \omega^{3}} \sum_{\vec{k}} \sum_{v c c^{\prime}} M_{v c}^{X} M_{c c^{\prime}}^{X} M_{c^{\prime} v}^{X} F_{v, c, c^{\prime}}(\omega) \\
F_{c c^{\prime} k}(\omega)=D_{c c^{\prime} k}(\omega, 2 \omega)+D_{c c^{\prime} k}(\omega,-\omega)+D_{c c^{\prime} k}(-\omega,-2 \omega) \\
D_{v, c, c^{\prime}}(\omega, 2 \omega)=\frac{1}{\left(E_{c v}(\vec{k})-i \gamma_{c v}(\vec{k})-2 \hbar \omega\right)\left(E_{c^{\prime} v}(\vec{k})-i \gamma_{c^{\prime} v}(\vec{k})-\hbar \omega\right)}
\end{gathered}
$$

$M_{v c}^{X}$ - factors represent momentum matrix elements for valence to conduction, conduction to upper conduction band transitions, $E_{c v}(\vec{k})$ is energy difference between bands, $\gamma_{c v}(\vec{k})$ is the interband polarization dephasing rate. Equations (3) and (4) estimate trends and features in $\chi_{2 D}^{(2)}$ dispersion due to different contributions. Namely, neglecting exciton effects and considering that primary contributions to the nonlinearity are due to interband transitions between two fully occupied valence bands and empty conduction bands. We have considered four parabolic energy bands near $K$-point of the Brillouin zone (BZ). 
Those include two valence bands with split off energy difference of $\Delta_{S O}=0.52 \mathrm{eV}$, with the effective masses of $m_{v}=0.52 m_{e}, m_{s o}=0.51 m_{e}$ and two conduction bands with the effective masses of $m_{c}=0.53 m_{e}, m_{c^{\prime}}=0.92 m_{e}$ and energy difference at K-point $\Delta_{c c^{\prime}}=1.79 \mathrm{eV}[6]$. We have also considered momentum matrix elements and dephasing rates are $k$-, and therefore energy, independent. The summation over $k$-states can be replaced by integral over energy that couples the resonant denominator in Equation (4) and joint density of states factor for the first conduction and top valence bands for continuum excitons $\left.\left(\sigma\left(E_{c v}\right) \approx\left(1-\exp \left(-\left(13.6 e V m_{R} / m_{e}\left(E_{c v}-E_{g}\right)\right)^{1 / 2}\right)\right) ; E_{c v}\right\rangle E_{g}\right)$. Figure 4.4 shows the results of our calculations when $\gamma$ was set to $35 \mathrm{meV}$. It is important to note that the nonlinearity is sensitive to a change of $E_{g}$. This is shown by comparing two simulations when $E_{g}$ is changed from $2.22 \mathrm{eV}$ (green curve) to $2.15 \mathrm{eV}$ (dash-dotted curve). It is apparent that when the bandgap is set at $\sim 2.2 \mathrm{eV}$, a better match with the experimental data around the peak area is obtained. We believe that band nonparabolicity becomes critical to explain the experimental data at higher photon energies. On the lower energy side, one finds that the rise in simulated $\chi^{(2)}$ is noticeably sharper when compared to our experimental data. The discrete exciton contributions can be represented mainly by resonant factors that are similar to the term in Equation (4) with peak energies $E_{n}=E_{0} /(n-1 / 2)^{2}$ used instead of $E(k)$. There are at least two clearly pronounced peaks in the experimental curve at photon energies of $2.64 \mathrm{eV}$ and $2.69 \mathrm{eV}$. The $50 \mathrm{meV}$ energy difference matches well for $n=2$ and $n=3$ excitons when the binding energy $4 E_{0}=0.71 \mathrm{eV}$ is assumed. Figure $4.4(\mathrm{~b})$ shows total nonlinearity due to the interband transitions and multiple exciton lines $(n=1-5)$ below the first conduction band 
states. The best fit is obtained for the exciton binding energy of $0.71 \mathrm{eV}$ while the bandgap $\left(E_{g}\right)$ parameter was set at $2.22 \mathrm{eV}$. The obtained value for the bandgap matches well with the ones reported in [5,6]. Exciton binding energy obtained from our data is also in close agreement with the value of $0.6 \pm 0.2 \mathrm{eV}$ reported by Wang et al. [5].

In conclusion, we have demonstrated precise measurements of the second order nonlinearity in atomically thin layer of semiconducting material within broad range of photon energies. Using ultra-broadband continuum pulses, we were able to detect fine features in the $\chi^{(2)}$ dispersion with high spectral resolution $(<3 \mathrm{meV})$. The nonlinearity onset is primarily due to monolayer $\mathrm{WSe}_{2}$ states that couple valence bands, excitonic levels and the continuum states above the first conduction band. Using our data, we estimate peak nonlinearity range for a sheet of $\mathrm{WSe}_{2}$ at $6.3-8.4 \times 10^{-19} \mathrm{~m}^{2} / \mathrm{V}$ and $932-1233$ $\mathrm{pm} / \mathrm{V}$ if we assume the existence of bulk properties. We also obtained from our data the room temperature bandgap and exciton binding energy that are estimated at $2.2 \mathrm{eV}$ and $0.7 \mathrm{eV}$ correspondingly. Our results show that the experimental $\chi^{(2)}$ dispersion data provide clear resolution of the near band-gap exciton states that are not clearly detected in linear spectroscopy methods.

Funding was provided by the National Science Foundation (NSF) (DBI-1355307) and University of Rhode Island start-up funds.

\section{References:}

${ }^{1}$ B. Radisavljevic, A. Radenovic, J. Brivio, V. Giacometti, and A. Kis, "Single-layer $\mathrm{MoS}_{2}$ Transistors," Nat. Nanotechnol. 6, 147-150 (2011). 
${ }^{2}$ N. Kumar, S. Najmaei, Q. Cui, F. Ceballos, P. M. Ajayan, J. Lou, and H. Zhao, "Second harmonic microscopy of monolayer $\mathrm{MoS}_{2}$," Phys. Rev. B 87, 161403-5 (2013).

${ }^{3}$ L. M. Malard, T. V. Alencar, A.P.M. Barboza, K. F. Mak, and A.M. de Paula, "Observation of intense second harmonic generation from $\mathrm{MoS}_{2}$ atomic crystals," Phys. Rev. B 87, 201401-5 (2013).

${ }^{4}$ E. Mishina, N. Sherstyuk, S. Lavrov, A. Sigov, A. Mitioglu,S. Anghel, and L. Kulyuk, "Observation of two polytypes of $\mathrm{MoS}_{2}$ ultrathin layers studied by second harmonic generation microscopy and photoluminescence," Appl. Phys. Lett. 106, 31901-5 (2015). ${ }^{5}$ G. Wang, X. Marie, I. Gerber, T. Amand, D. Lagarde, L. Bouet, M. Vidal, A. Balocchi, and B. Urbaszek, "Giant Enhancement of the Optical Second-Harmonic Emission of $\mathrm{WSe}_{2}$ Monolayers by Laser Excitation at Exciton Resonances,"Phys. Rev. Lett. 114, 097403-6 (2015).

${ }^{6}$ A. Ramasubramaniam, "Large excitonic effects in monolayers of molybdenum and tungsten dichalcogenides,” Phys. Rev. B 86, 115409-6 (2012).

${ }^{7}$ V. G. Dmitriev, G. G. Gurzadyan, Handbook of Nonlinear Optical Crystals (Springer Series in Optical Sciences), 2d Edition, (1996).

${ }^{8}$ N. Bloembergen, P.S. Pershan , "Light Waves at the Boundary of Nonlinear Media ," Phys. Rev. 128, 606-622 (1962).

${ }^{9}$ M. Merano, "Nonlinear Optical Response of a Two-dimensional atomic crystal," Opt. Lett. 41,187-190 (2016).

${ }^{10}$ M. Merano, "Fresnel coefficients of a two-dimensional atomic crystal," Phy. Rev. A 93, 013832 (2016) 
${ }^{11}$ G.D. Boyd, D.A.Kleinman, "Parametric interaction of Focused gaussinan light beams ," J. Appl. Phys. 39, 3597-3639 (1968).

${ }^{12}$ Supplement

${ }^{13}$ Y.Li, Y.Rao, K.F.Mak, Y.You, S.Wang, C.R.Dean, and T.F.Heinz,“ Probing symmetry properties of Few-layer $\mathrm{MoS}_{2}$ and h-BN by optical second-harmonic generation," Nano Lett. 13, 3329-3333 (2013).

${ }^{14}$ S.M. Eichfeld, C.M.Eichfeld, Y-C. Lin, L. Hossain. J.A.Robinson, "Rapid, nondestructive evaluation of ultrathin WSe2 using spectroscopic ellipsometry," APL Mater. 2, 092508 (2014).

${ }^{15}$ I. Shoji, T. Kondo, A. Kitamoto, M. Shirane, and R. Ito, “Absolute scale of secondorder nonlinear-optical coefficients,'J. Opt. Soc. Am. 14, 2268-2294 (1997 ${ }^{16}$ K. Vodopyanov, O. Levi, P.Kuo, T. Pinguet, J.Harris, M.Fejer, B. Gerard, L. Becouarn, and E. Lallier, "Optical parametric oscillation in quasi-phase-matched GaAs" Opt. Lett. 29, 1912-1914 (2004) .

${ }^{17}$ M. L. Trolle, G. Seifert, T. G. Pedersen ,"Theory of excitonic second harmonic generation in monolyaer $\mathrm{MoS}_{2}$," Phys. Rev B 89, 235410 (2014).

${ }^{18}$ R. Leitsmann, W.G.Schmidt, P.H.Hahn, and F.Bechstedt, "Second-harmonic polarizability including electron-hole attraction from band-structure theory," Phys. Rev. B 71, 195209 (2005). 

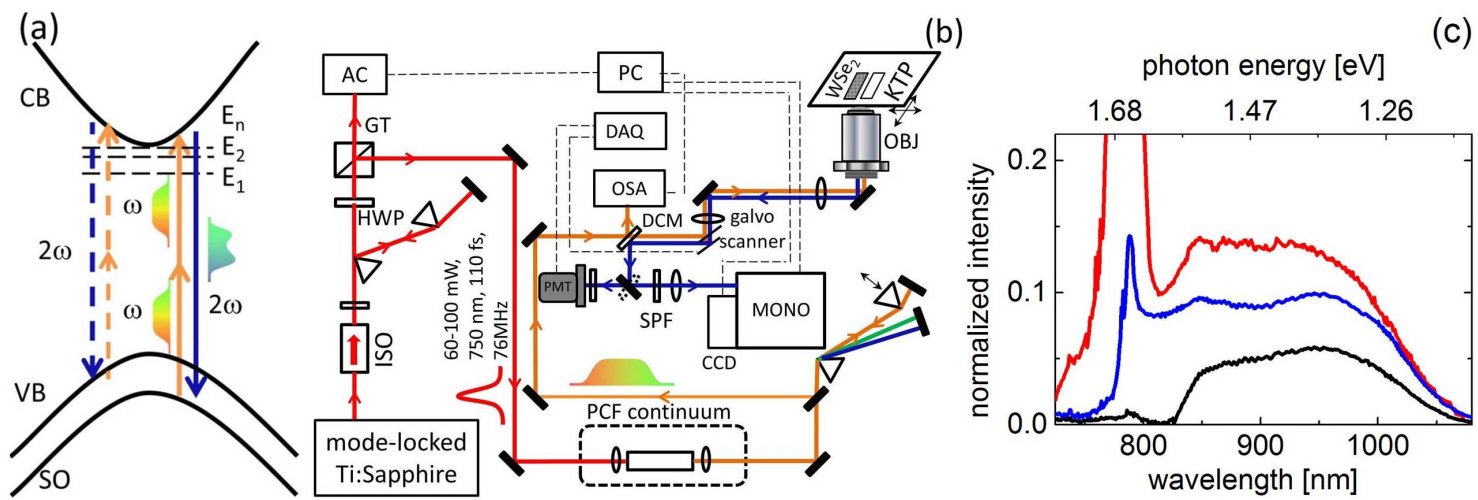

Figure 4.1: (a) Photon diagram SHG process in monolayer semiconductor. (b) Setup: ISO-optical isolator, PCF - photonic crystal fiber, DCM- dichroic mirror, OBJ-high numerical aperture objective lens, SPF-short pass filter. Broadband near-IR pulse derived from femtosecond continuum serves as fundamental beam. Spectra of the generated second harmonic pulses carrying resonant features are analyzed. (c) Fundamental spectra.
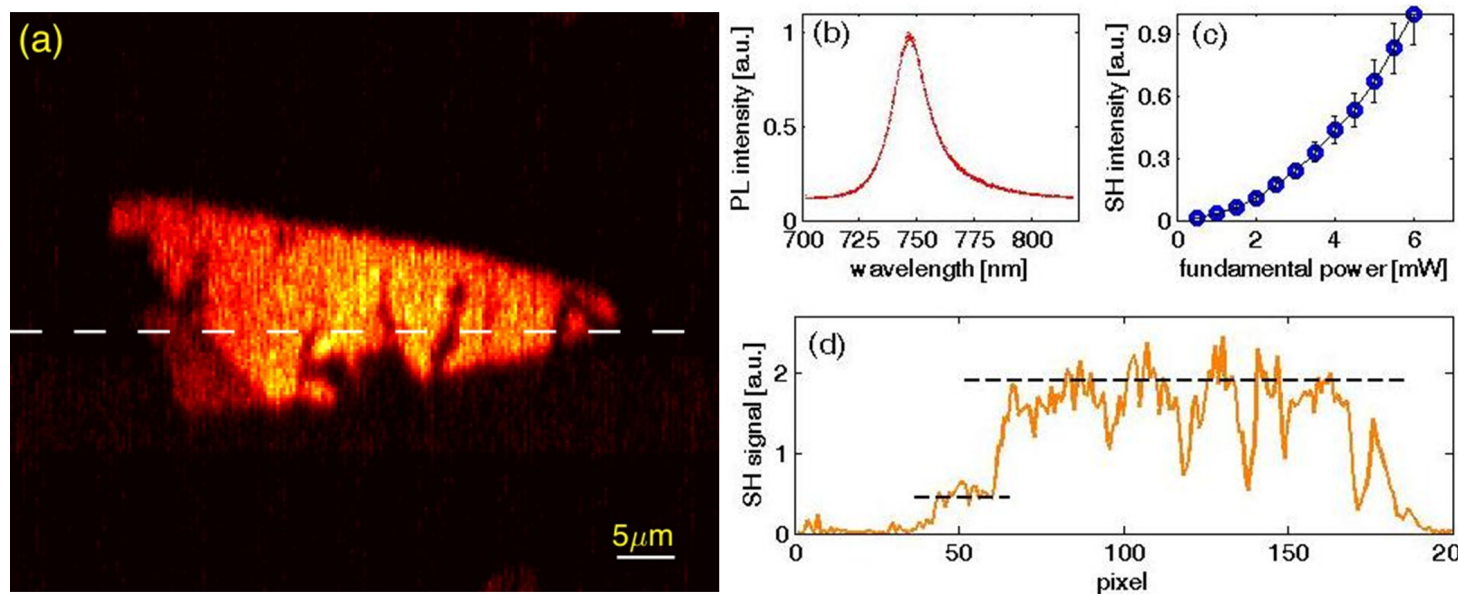

Figure 4.2: (a) SHG image of a single layer $\mathrm{WSe}_{2}$ flake. Lower left corner of the sample contains three $\mathrm{WSe}_{2}$ layers and therefore show four times smaller signal. (b)

Photoluminescence data obtained from the flake when it was excited by $532 \mathrm{~nm}$ continuous wave beam, (c) detected second harmonic beam power versus power of the fundamental beam, (d) SHG signal change across the image along the dashed line. 

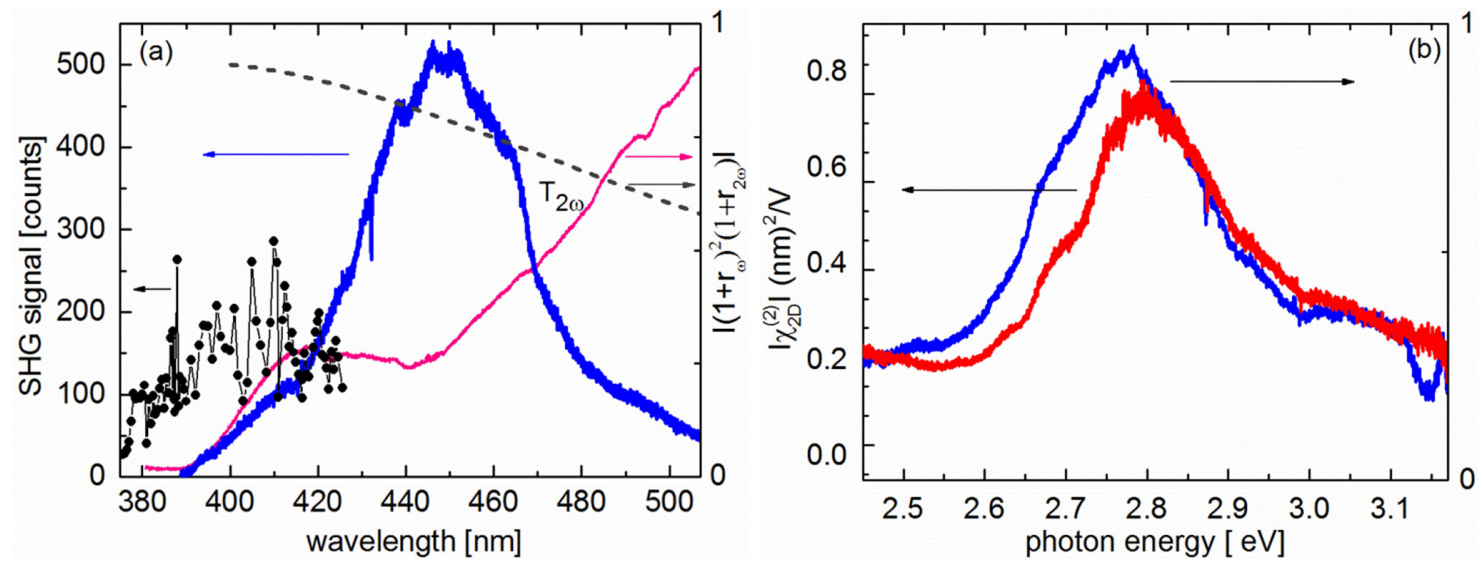

Figure 4.3: (a) Second harmonic signal spectrum $\left(P_{2}^{2 D}\right)$ detected from monolayer part of $\mathrm{WSe}_{2}$ sample (blue), relative transmission ( $\left.T_{\omega}\right)$ of the optical system versus wavelength (cyan), calculated enhancement factor $\zeta$ (black dash); (b) resulting absolute value of the second order nonlinearity $\left(\chi^{(2)}\right)$ obtained by power relationship (blue curve) and the comparative method using KTP crystal (red curve).


Figure 4.4: (a) Normalized $\chi_{2 D}^{(2)}$ versus SH photon energy (black curve). Green solid curve is the result of $\chi_{2 D}^{(2)}$ calculations for $E_{g}=2.21 \mathrm{eV}$ while changing the parameter to $2.15 \mathrm{eV}$ results in the blue dash-dotted curve. (b) Experimental $\chi_{2 D}^{(2)}$ dispersion (black 
line) and result of calculations accounting for contributions due to exciton states with energies $E_{n}$ below the bandgap. Colored (dashed) lines show contributions to the nonlinearity from broadened ( $\Delta v_{n}=35-42 \mathrm{meV}$ ) exciton lines with the main quantum numbers $n=2-5$. 


\section{APPENDIX A.}

\section{SOURCES OF LIGHT AND SUPERCONTINUUM GENERATION}

\section{A.1. Pump laser and seed DBR laser}

In the SBS laser experiment there were two different light sources. The first of which was a 5W 14 pin cooled multimode pump laser diode (EM4). This laser diode had a center wavelength of $976 \mathrm{~nm}$, was fiber coupled with a fiber NA of 0.22 and cladding pumped the Yb-fiber in the laser cavity. A laser diode is an electrically pumped semiconductor laser in which a current-carrying $\mathrm{p}-\mathrm{n}$ junction acts as a gain medium. This type of laser is ideal for several applications where brightness is essential.

The second type of light source in this experiment was a 1064nm 14 pin DBR laser (EM4). This model had a 150mW output power and a laser linewidth of $10 \mathrm{MHz}$. It contains a cooler, thermistor and monitor detector. A distributed Bragg reflector (DBR) is an InGaAs quantum well laser diode and is ideal in applications where mode stability, low RIN and stable PM properties are needed. We used this laser as a seeder for our fiber laser injection setup.

\section{A.2. Mode-locked Ti:sapphire laser}


The primary laser source used in the CARS experiments was the mode-locked Ti:sapphire ultrafast laser (Mira-HP, Coherent) which was tunable from 700 to $1000 \mathrm{~nm}$ and had an output power of $\sim 3-3.7 \mathrm{~mW}$. The mode-locked laser was pumped by a green laser with output power $17 \mathrm{~W}$ and a repetition rate (frequency) of $76 \mathrm{MHz}$. The pulsed output light is passed through a two-cavity prism to compensate the pulse. The majority of the beam is split into two equal parts and synchronously pump the two optical parametric oscillators (OPO) and the remainder of the laser emission is used as the probe pulsed light for the coherent anti-Stokes Raman spectroscopy (CARS) experiments.

\section{A.3. Optical parametric oscillators (OPO)}

Two OPOs were used simultaneously in the aforementioned publications. The first OPO setup involves an optical resonator containing a stoichiometric lithium tantalite (PPSLT) nonlinear crystal as a nonlinear gain media. The nonlinear crystal allows for quasi-phase matching condition. The OPO generates parametric oscillation at the near-IR pump wavelengths for both continuous wave $(\mathrm{CW})$ and pulsed mode. The nonlinear crystal has $1 \mathrm{~mm}$ width, $0.5 \mathrm{~mm}$ thickness and $15 \mathrm{~mm}$ length. The grating period is varied from 17.5 to $24.8 \mu \mathrm{m}$ with $0.6 \mu \mathrm{m}$ differences between consecutive periods. The pump power, delivered by the TiS mode-locked laser, was $1.15 \mathrm{~mW}$ and was focused onto the nonlinear crystal by a $76 \mathrm{~mm}$ focal length lens. The OPO cavity consists of two concave mirrors and three parallel substrate mirrors. A pair of Brewster cut angle prisms was used to compensate for the dispersion. The optimal distance between prisms (apex to 
apex), which generates short pulses within the tuning range $960-1050 \mathrm{~nm}$, was found to be $280 \mathrm{~mm}$. The pulse characteristics are shown in Figure A.1.

The second OPO is based on a periodically pooled lithium niobate (PPLN) nonlinear crystal with a similar setup as the first OPO. This OPO is capable of generating wavelengths ranging from 1050 to $1100 \mathrm{~nm}$.

\section{A.4. Supercontinuum generation}

The broadband continuum pulses needed for the SHG setup are a result of the generation of the supercontinuum from a photonics crystal fiber (PCF). Supercontinuum generation produces ultra-broadband optical spectrum when pumped by a high-power laser source. In our experimental configuration, the pump laser source is composed of femtosecond pulses. A photonic crystal, with core diameter $1.2 \mu \mathrm{m}$ and zero group dispersion at $750 \mathrm{~nm}$, was used in order to produce broadband $(450-1150 \mathrm{~nm})$ continuum. A characteristic spectrum is given in Figure A.2.

The PCF was installed on an XYZ stage (466A XYZ fixture, Newport Corp.), aligning the PCF marked polarization with the polarization of the Ti:sapphire beam. This alignment was completed with the use of a half-wave plate on a rotating mount to change the linear polarization angle and a Glan-Thompson polarizer to make sure the input polarization was vertical. The XYZ stage incorporated a 40x microscopic objective lens to effectively couple light into the fiber. The input power into the PCF did not exceed 50 $\mathrm{mW}$ in order to avoid damaging the fiber during the light coupling adjustments. Once the continuum pulse was successfully observed, the input power was increased to $100 \mathrm{~mW}$ 
and the translational stage and steering mirrors were tuned to optimize the effect. At this time, an objective lens (20x, Newport Corp.) was installed and adjusted to collimate the output beam. It is important to note that the properties of the generated continuum pulses are defined by the amount of coupled power rather than the coupling frequency. The supercontinuum generation setup is shown in Figure A.3. 

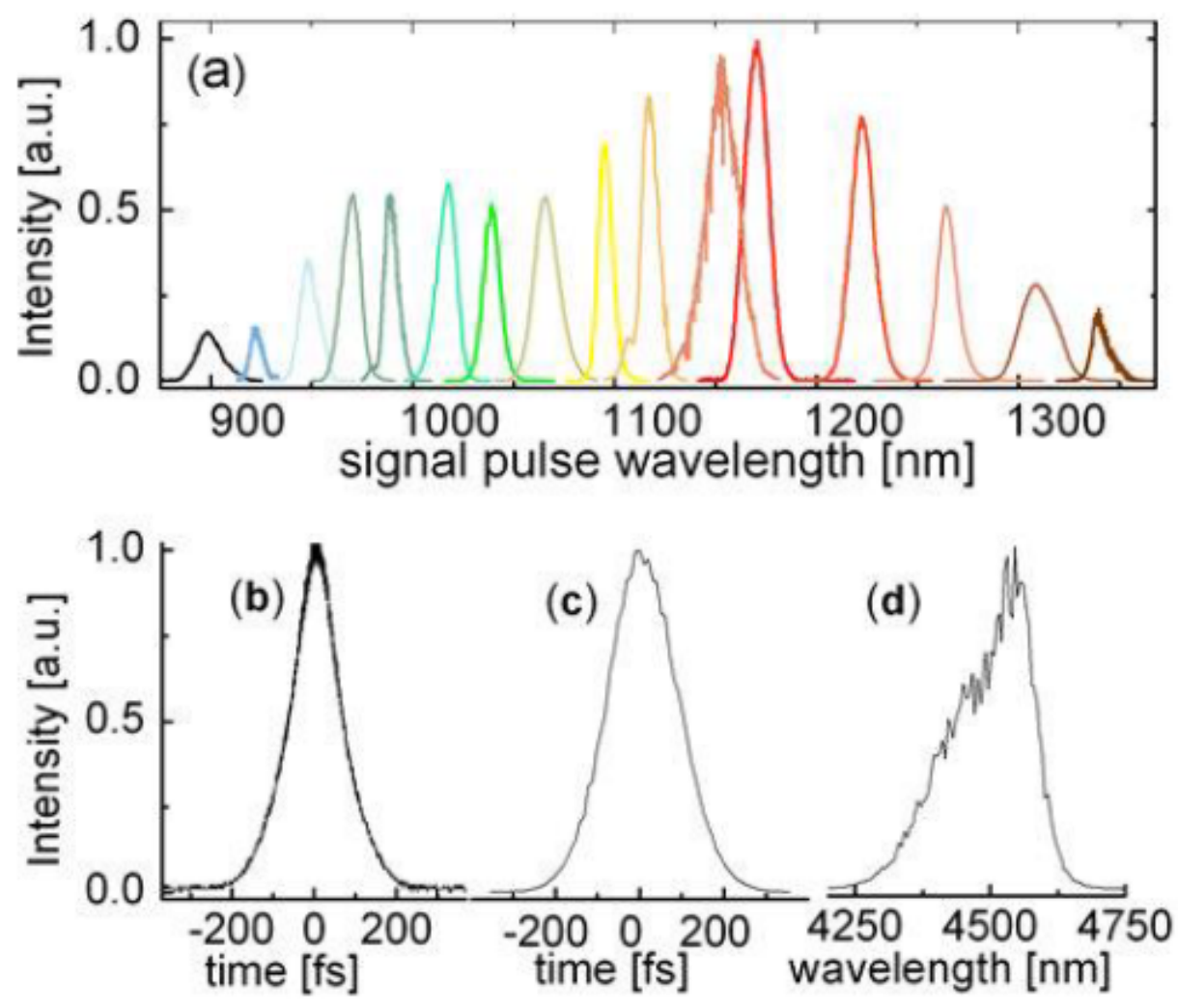

Figure A.1: OPO output pulse characteristics: (a) shows OPO pulse spectra obtained throughout available tuning range for the OPO. (b) (c) Shows typical pulse autocorrelations for signal (b) and idler (c) beams. (d) Shows idler pulse spectrum at $\sim 4.5$ $\mu \mathrm{m}$ while OPO can still deliver $\sim 50 \mathrm{~mW}$ of average power in idler branch. 

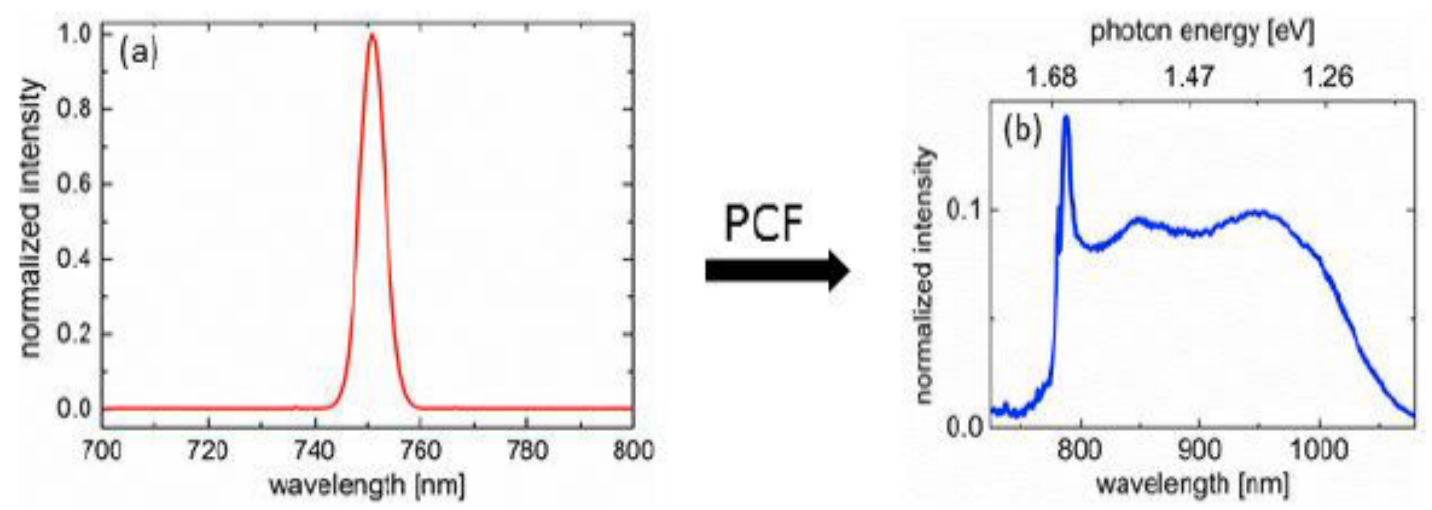

Figure A.2: The characteristic curve of a broadband pulse generated by PCF: (a)

Fundamental pulse of TiS at $750 \mathrm{~nm}$. (b) The output broadband pulse when the $750 \mathrm{~nm}$ pulse with energy $150 \mathrm{~mW}$ passes through PCF.

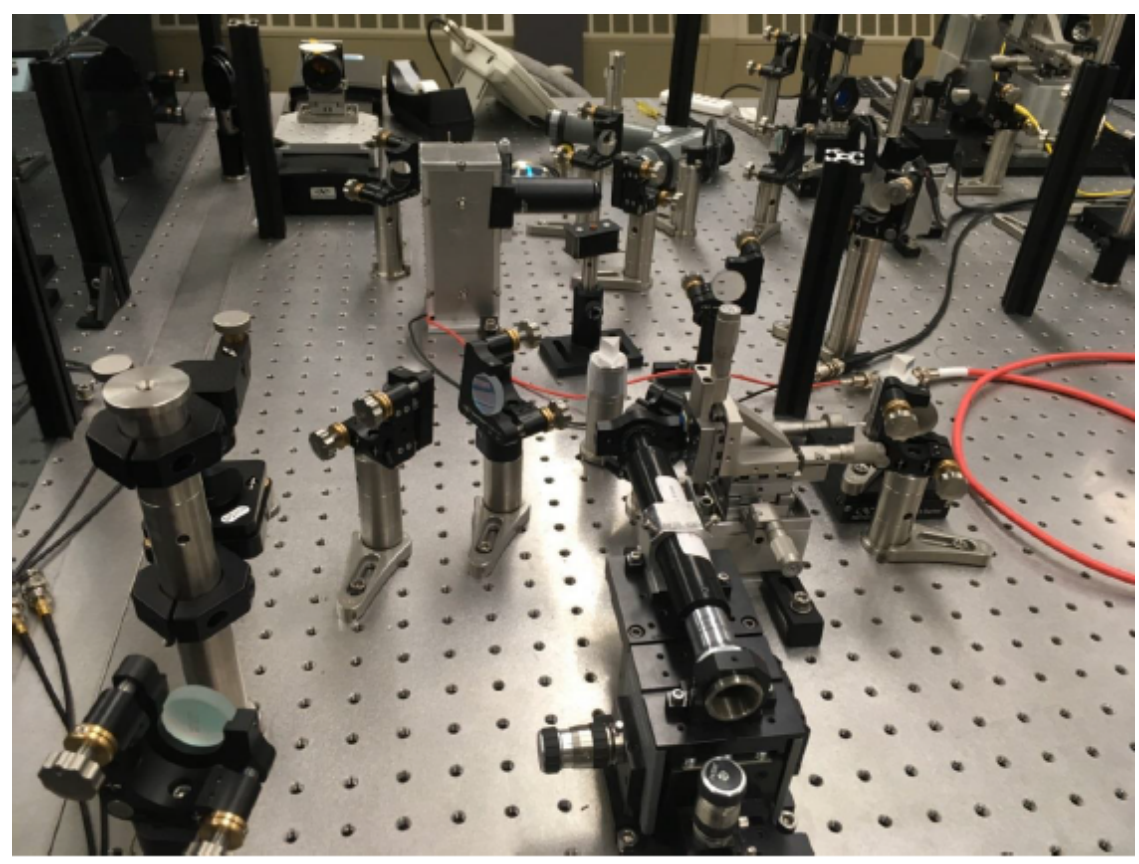

Figure A.3: Actual setup configuration for the supercontinuum generation setup. 


\section{APPENDIX B.}

\section{EXPERIMENTAL TECHNIQUES AND PROCEDURES}

\section{B.1. Chapter 4 Supplement}

The electric field for the incident beam at fundamental frequency is represented by the following expression:

$$
E_{1}=\frac{E_{01}}{2}\left(e^{i \omega t}+e^{-i \omega t}\right)
$$

In this equation, we have used the SI system of units. The intensity is then written as:

$$
\begin{aligned}
& I_{1}=\frac{1}{2} \varepsilon_{0} c\left|E_{01}\right|^{2} \\
& E_{01}(x, y)=E_{0} e^{-\frac{x^{2}+y^{2}}{w_{0}^{2}}}
\end{aligned}
$$

The second harmonic pulse peak power generated in bulk crystal under tight Gaussian focusing conditions can be represented by the expression that follows from formula (2.58) under (2.99) (p.3609 Ref. [11]):

$$
P_{2}=\frac{8 \pi \omega_{1}^{2}}{\varepsilon_{0} c^{3} n_{2}} \frac{1}{\Theta^{2}}\left|d_{e f f}\right|^{2} P_{1}^{2}
$$

$\mathrm{n}_{2}$ - refractive index at $\mathrm{SH}$ frequency, $\mathrm{Q}$ - numerical aperture of the microscope objective. Given the fact that the incident and $\mathrm{SH}$ beams are partially reflected off the entrance and exit facets correspondingly, we write: 


$$
P_{2 \omega}^{B}=\frac{8 \pi \omega_{1}^{2}}{\varepsilon_{0} c^{3} n_{2}} \frac{1}{\Theta^{2}}\left|\chi^{(2)}\right|^{2}\left(\frac{2}{n_{1}+1}\right)^{4}\left(\frac{2}{n_{2}+1}\right)^{2} P_{\omega}^{2} \cong \frac{8 \pi \omega_{1}^{2}}{\varepsilon_{0} c^{3} n}\left(\frac{2}{n+1}\right)^{6} \frac{1}{\Theta^{2}}\left|d_{e f f}\right|^{2} P_{1}^{2}
$$

We have neglected the dispersion in the crystal (i.e. $\mathrm{n}_{2} \approx \mathrm{n}_{1}=$ n) in order to arrive at Equation (4). Also, we have used $w_{0} \approx l / p Q$ for high NA objective.

The solution for the SH field in the 2D layer and the corresponding intensity $\left(I_{2}\right)$ can be obtained by using Equation (12) in Ref. [9]. Both beams (SH and the fundamental) have the Gaussian spatial distribution and the field's amplitude can be represented via fundamental pulse peak power using:

$$
P_{2}^{2 D}=(2)^{-1} n_{2} \varepsilon_{0} c \zeta^{2} \int\left|E_{01}^{2}(x, y)\right|^{2} d x d y
$$

and

$$
\left|E_{0}\right|^{2}=\frac{4 P_{1}}{\pi \varepsilon_{0} c w_{0}^{2}}
$$

The latter relationship is obtained by integrating Equation (2) over space. The complex factor represented by:

$$
\zeta=\left|\left(1+r_{\omega}\right)^{2}\left(1+r_{2 \omega}\right)\right|
$$

accounts for the interference effect in the two-layered substrate for both fundamental and SH beams $[9,10]$.

Following the solution for the electric field at SH generated in 2D material [9] and taking into account Equations (5) and (6), the peak power at $\mathrm{SH}$ frequency $\left(\mathrm{P}_{2}{ }^{2 \mathrm{D}}\right)$ is:

$$
P_{2}^{2 D}=\frac{2 P_{1}^{2} k_{1}^{2}\left|\chi^{(2)}\right|^{2}\left|\left(1+r_{\omega}\right)^{2}\left(1+r_{2 \omega}\right)\right|^{2}}{n_{1}^{2} \pi \varepsilon_{0} c w_{0}^{2}}
$$


Then, the ratio $(\rho)$ of the two peak powers (2D sheet/bulk) is:

$$
\rho=\frac{P_{2}^{2 D}}{P_{2 \omega}}=\frac{\zeta^{2} n(n+1)^{6} \Theta^{2}\left|\chi_{2 D}^{(2)}\right|^{2}}{256 \pi^{2} w_{0}^{2}\left|d_{e f f}\right|^{2}}
$$

And

$$
\chi_{2 D}^{(2)}=\frac{16 \lambda \sqrt{\rho}}{\zeta(n+1)^{3} \sqrt{n} \Theta^{2}}\left|d_{e f f}\right|
$$

This equation is represented as Equation (2) in the manuscript. It is worth noting that in Equation (10), $\chi_{2 D}^{(2)}$ sheet second order nonlinearity is measured in $\mathrm{m}^{2} / \mathrm{V}$.

The following work corresponds to the estimate for the nonlinearity $\chi_{2 D}^{(2)}[\mathrm{m} / \mathrm{V}]$, when the 2D layer is treated as bulk-like system. For a nonlinear interface, the solution for the electric field at SH frequency is given [8] by:

$$
E_{2}=\frac{-4 P^{(2)} \xi_{1}^{\prime}}{\varepsilon_{0}\left(n_{2}+1\right)\left(n_{2}+n_{1}\right)\left(n_{1}+1\right)^{2}}
$$

$\mathrm{n}_{2}, \mathrm{n}_{1}$ stand for the refractive indices (at SH and fundamental frequencies, respectively) of the bulk-like $\mathrm{WSe}_{2}$ thin layer material with the actual numerical values for different wavelengths (optical frequencies) provided in [14]. Furthermore, we write:

$$
\begin{gathered}
P^{(2)}=\varepsilon_{0} \chi_{2 D}^{(2)}{ }^{\prime} E_{1}^{2} \\
\zeta_{1}{ }^{\prime} \cong 1+r_{23}\left(1-r_{W}\right)\left(1-r_{W}{ }^{\prime}\right) e^{-i 2 \phi} \\
r_{23}=\frac{\bar{n}_{3}-\bar{n}_{2}}{\bar{n}_{3}+\bar{n}_{2}} ; r_{W}=\left(\frac{n_{2}-1}{n_{2}+1}\right) ; r_{W}{ }^{\prime}=\left(\frac{n_{2}-\bar{n}_{2}}{n_{2}+\bar{n}_{2}}\right) \\
\phi=\frac{2 \pi}{\lambda_{2}} \bar{n}_{2} t
\end{gathered}
$$


$\bar{n}_{2}, \bar{n}_{3}$ - refractive indices of $\mathrm{SiO}_{2}$ (subscript 2) and $\mathrm{Si}$ (subscript 3 ) at $\mathrm{SH}$ freq, $t$ thickness of $\mathrm{SiO}_{2}$ layer.

$$
\begin{gathered}
P_{2}^{2 D^{\prime}}=\frac{32\left|\chi_{2 D}^{(2)}\right|^{2} P_{1}^{2}\left|\zeta_{1}\right|^{2}}{\pi \varepsilon_{0} c w_{0}^{2} f\left(n^{\prime}\right)} \\
\sqrt{f\left(n^{\prime}\right)}=\left(n_{2}+1\right)\left(n_{2}+n_{1}\right)\left(n_{1}+1\right)^{2}
\end{gathered}
$$

Dividing $P_{2}^{2 D}$ (Equation (16)) into $P_{2}$ (Equation (4)) yields:

$$
\left|\chi_{2 D}^{(2)}\right|=\sqrt{\rho} \frac{8 \pi \sqrt{f\left(n^{\prime}\right)}}{\sqrt{n}(n+1)^{3} \Theta^{2}|\xi|}\left|\chi_{B}^{(2)}\right|
$$

\section{B.2. Seeded SBS Q-switched fiber laser based on SBS procedure}

There were four different procedural components in this setup: the SBS laser operation procedure, the injection waveform generation procedure, the injection/fiber core coupling procedure and the procedure for running the experiment itself. In the SBS laser operation procedure the fiber position first needed to be optimized. This was done by free-space coupling the multi-mode pump laser through an aspheric lens and into the core and inner cladding of the active fiber. The fiber end and the aspheric lens were positioned on an $\mathrm{XYZ}$ translational stage controlled by three spatial micrometers and had a rotating fiber holder used to match the vertical polarization of the injected light mode with the PANDA fiber slow-axis (see Figure B.1). The pump laser diode was initially set to have a low current $(400 \mathrm{~mA})$ and a power meter was put on the opposite side of the fiber measuring how much pump light was propagating through the fiber. The micrometers were adjusted until the power meter measured the maximum throughput 
power. Because the active fiber was double-clad fiber, the pump light didn't have to couple into the narrow fiber core. Instead, the pump light was coupled into the inner cladding waveguide $(125 \mu \mathrm{m}$ diameter) and upon contact with the ytterbium ions in the doped single-mode core, the light was almost immediately absorbed creating population inversion in the active fiber. Cladding-pumping is a critical property of high-power fiber lasers and amplifiers and is shown schematically in Figure B.2 (a). A relevant property of double-clad fiber is that if the fiber termination is perpendicularly cleaved, $4 \%$ Fresnel reflection of the outgoing beam will act as feedback into the single-mode fiber core but if the fiber termination is angle cleaved (typically less than 10 degrees) the reflection will propagate into the inner cladding and not back into the fiber core. This property is illustrated in Figure B.2 (b). If an HR mirror or a partially reflecting mirror is used in the cavity configuration, a power meter is setup after the dichroic mirror, the pump laser current is increased to a current large enough to generate lasing but not large enough to initiate SBS pulsing (typically approximately $1000 \mathrm{~mA}$ ) and the mirror is tuned until the maximum output power is measured. The XYZ translational stage holding an aspheric lens in front of the fiber-coupled fast photodetector is then tuned to see the laser traces on the oscilloscope.

Once the SBS laser has been optimized, the injection components need to be setup. First, the seed DBR laser is turned on with a moderately high current $(220 \mathrm{~mA})$ and the emitted light is free-space coupled into the input fiber of the Mach-Zehnder modulator. This input light coupling is optimized with micrometers until a power meter registers a maximum amount of power exiting the modulator output fiber. The modulator had to then warm up for a half-hour or else the exiting light power would drift. Once 
warmed up, the Mach-Zehnder modulator could operate properly without any drift detected. The arbitrary function generator drove the RF signal into the modulator generating the waveform and the DC bias voltage applied to the modulator was at the quadrature (half max) point for proper waveform injection generation (see Figure B.3). The output fiber is connected to a fiber-collimator and then the collimator is rotated in its mount to assure that the exiting light is vertically polarized. A two-lens system was constructed with a moving translational stage under the far lens to narrow to the beam waist. This light then reflected off of a two-mirror system to direct the beam path and couple as much optical power possible through the free-space isolator. As the injection light exits the isolator and passes through the 50/50 beamsplitter, the beam path is tuned slightly to make the light incident on a photodetector.

In order to effectively couple the injection light into the fiber core (and not the inner cladding), we first setup and tuned a diffraction grating as the cavity back mirror in the Littrow configuration. Tuning the grating required injecting CW light of $1064 \mathrm{~nm}$ wavelength and adjusting the angles of the grating until the output power after the 50/50 beamsplitter was at a maximum level. The grating configuration is schematically depicted in Figure B.4. Once the grating was configured, the injection light beampath and the laser output beampath were overlapped by having both beams pass through two irises separated by approximately two feet of open table space. The pump laser was set to a low current which would generate somewhat inverted fiber but not strong enough to initiate lasing and a $10 \mathrm{kHz}$ sine wave was injected into the fiber. The reflected amplified sine wave was then measured by the fast photodetector and the oscilloscope. The beamsplitter and other mirrors were tuned in order to generate the most amplified sine 
wave on the oscilloscope as possible and then the pump current was adjusted lower until the sine wave was no longer visible to show that the injection successfully passes through the fiber core and not passing through the inner cladding which would not show an amplification property with the varying pump laser current.

Once the injection waveform light was successfully coupled into the fiber core, we could run the experiment. The SBS laser output was blocked and the pump current was set to its maximum current used in the experiment (typically 3.6A to 4A) and the laser warmed up for 10 minutes before any measurements were taken. After this time, the injection light was blocked and the SBS laser was unblocked and free-running SBS oscilloscope traces were recorded. The injection was then unblocked and the SBS laser output was then measured on the oscilloscope, on the optical spectrum analyzer and the power meter. Typical pulse trains and individual SBS pulses were both measured in this fashion.

\section{B.3. Coherent anti-Stokes Raman spectroscopy (CARS) procedure}

In the CARS procedure, two coherent light beams with frequencies $\omega_{1}$ and $\omega_{2}$ can be used to drive a Raman vibrational mode at frequency $\omega_{R}=\omega_{1}-\omega_{2}$. The two beams are overlapped in time and space to effectively generate this effect. A third pulse of frequency $\omega_{3}$ with a variable time delay is then injected into the Raman excited medium generating a CARS signal with an up-shifted, anti-Stokes frequency $\omega_{3}+\omega_{\mathrm{R}}$. The experimental configuration is shown in Figure B.5. The two pulses needed to coherently drive lattice vibrations within a sample's volume were provided by synchronously 
pumped OPOs running at $76 \mathrm{MHz}$. The OPOs were pumped by a split-output highpower mode-locked Ti:sapphire oscillator tuned to $765 \mathrm{~nm}$ and had output frequencies in the ranges of $970-1020 \mathrm{~nm}$ and $1050-1100 \mathrm{~nm}$. The third pulse was a lower intensity probe pulse that consisted of a small portion of the mode-locked laser and had a variable time delay. These three pulses were intrinsically synchronized and focused by a high numerical aperture (NA 1.25) objective lens. An image of the actual CARS setup is shown in Figure B.6.

The CARS measurement technique used a high numerical aperture (NA $\sim 0.9$ ) condenser (Olympus, model UplanSApo-60x/1.20 W IR) followed by a diffraction grating and a set of bandpass filters. This equipment allowed for the efficient detection of the signal of interest on the background of other signal light generated within the focal volume. A photomultiplier tube (PMT) with high gain and quantum efficiency (Hamamatsu, model R10699) was used to detect anti-Stokes signal photons at selected wavelengths and the PMT current output was digitized by a high-speed data acquisition card (DAQ, model NI-6361). Using this experimental arrangement, we can routinely detect CARS signal versus probe pulse delay times within five decades. An image of the signal detection system for the CARS procedure is shown in Figure B.7.

Special attention had to be paid to making an initial zero time delay for the CARS signal whereby the three light sources had spatial and temporal overlap. In this effort, two irises were placed after the dichroic mirror and the beams were guided through the center of both irises. To setup the correct timing between the beam overlap, the optical path lengths of each beam was first measured taking care to include the thickness of any optic along the beam path and the translational stages for the delay line. 
The method of finding the zero-delay point involved the use of a Beta-Barium Borate (BBO) nonlinear optical crystal to observe the sum frequency generation (SFG) signal resulting from the overlap of the input beams. Initially, both OPOs were blocked and the Ti:sapphire laser was focused through the BBO crystal. Then, the crystal was vertically rotated and the output light was the wavelength corresponding to the color purple and the crystal was slightly rotated to optimize the signal. Once this has been accomplished, the Ti:sapphire light was blocked and the two OPOs were unblocked. The crystal is then rotated displaying two light beams resulting from the second harmonic signals of the OPOs at two different crystal positions. The crystal position is then set between the two second harmonic signal spots. The mirror on the translational stage was moved backward and forward in order to detect a mixing signal. If one of the OPOs is blocked, the signal disappears. This implies that there is zero time delay for the two OPOs. At this point, one OPO is blocked and the Ti:sapphire light is unblocked. The same procedure as was used for the alignment of the two OPOs was employed to line up the Ti:sapphire and either OPO light. At this point, the three beams are in zero time delay. To test this outcome, if we block one of the OPO light sources one of the output spots will disappear and if the other OPO is blocked, the other spot will disappear. During this procedure, the power of OPO- 1 was set at $56 \mathrm{~mW}$ and $200 \mathrm{~mW}$ for OPO-2 thus yielding a combined power of $256 \mathrm{~mW}$ after DCM1.

\section{B.4. Second harmonic generation (SHG) microspectroscopy procedure}


SHG is the nonlinear process where an input wave composed of a short optical pulse with frequency $\omega$ passes through a nonlinear material lacking inversion symmetry and generates a pulse wave with twice the initial optical frequency $2 \omega$. SHG is also known as frequency doubling and is a special type of the nonlinear process sum frequency generation (SFG). A schematic diagram of SHG is given in Figure B.8.

The experimental setup used in the SHG microspectroscopy procedure employed a high-repetition rate femtosecond Ti:sapphire laser with a central wavelength tuned to $750 \mathrm{~nm}$. This light was coupled into a photonic crystal fiber (PCF) with a core diameter of $1.2 \mu \mathrm{m}$ utilized a $40 \mathrm{x}$ objective lens $(\mathrm{NA}=0.75)$ to generate a spectrally stable continuum in the range of $\sim 450 \mathrm{~nm}$ to $\sim 1200 \mathrm{~nm}$ (UV to IR light spectrum) with a total power of approximately $45 \mathrm{~mW}$. The power of the incident beam coupled into the PCF was approximately $100 \mathrm{~mW}$. The generated continuum was dispersed by a pair of prisms and then a portion of the spectrum, having a fairly smooth envelope in the wavelength range of interest, was selected as the fundamental beam. The beam was angle scanned with a scanning area of approximately $200 \times 200 \mu \mathrm{m}^{2}$ in the image plain of the objective. Samples in this procedure were positioned using a micrometer driven translational stage. A schematic diagram of the SHG experimental setup is shown in Figure 4.1 in Chapter 4.

The SHG microspectroscopy measurement procedure began with taking the spectral and power measurements of the incoming fundamental beam before the objective. This was done with a power meter and an optical spectrum analyzer (Anritsu, model MS 9710C). After these measurements, the second-harmonic signal from the sample was collected through the same objective in the backward direction and filtered out with a dichroic mirror and shortpass filter (SPF) before entering a calibrated grating 
monochromator (Horiba, iHR320). The monochromator had a cooled, sensitive CCD detector (Horiba, model Syncerity-356399). The SHG signal beam was additionally sent to a photomultiplier tube (PMT) to enable imaging of the sample. Data acquisition was performed using a data acquisition card as well as with the monochromator's USB interface. Both experimental components were controlled with the LabVIEW interface software. 


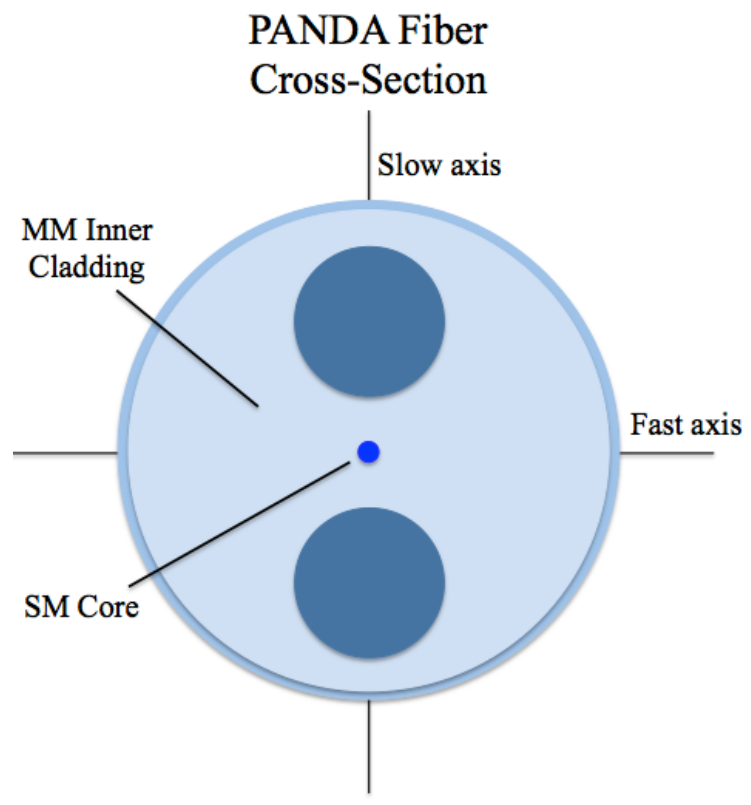

Figure B.1: Typical cross-sectional geometry of a PANDA fiber. The core is a singlemode waveguide and the inner cladding is multi-mode waveguide. The stress induced birefringence (dark blue) allows for polarization maintaining properties for linearly polarized light aligned with the slow-axis.

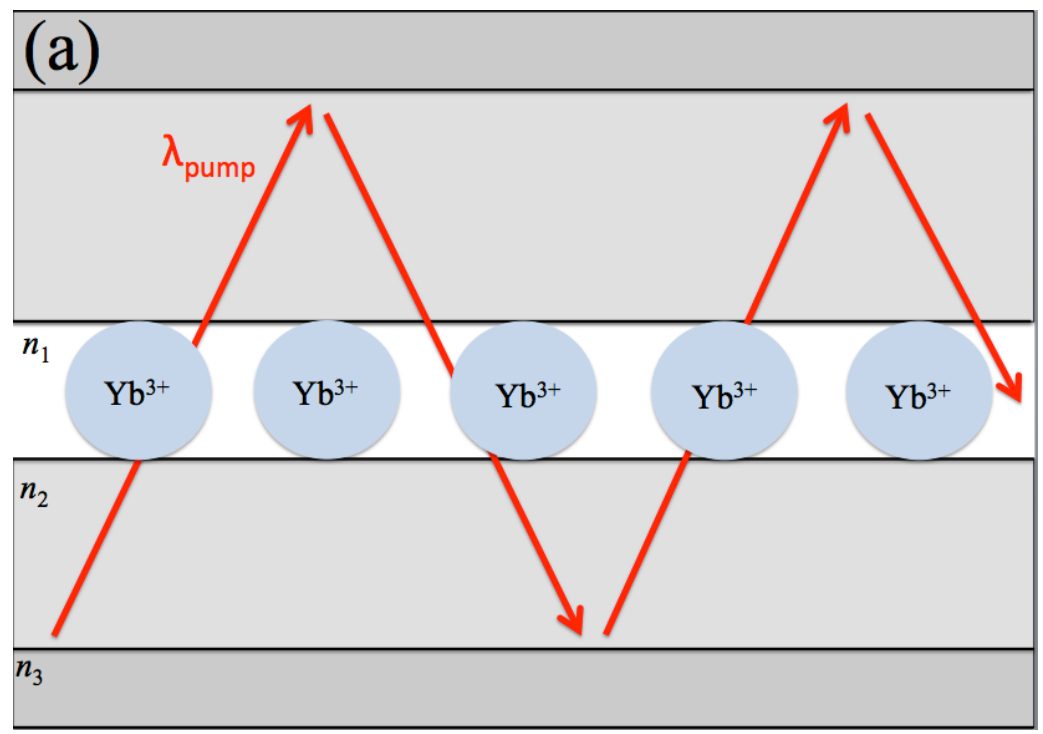




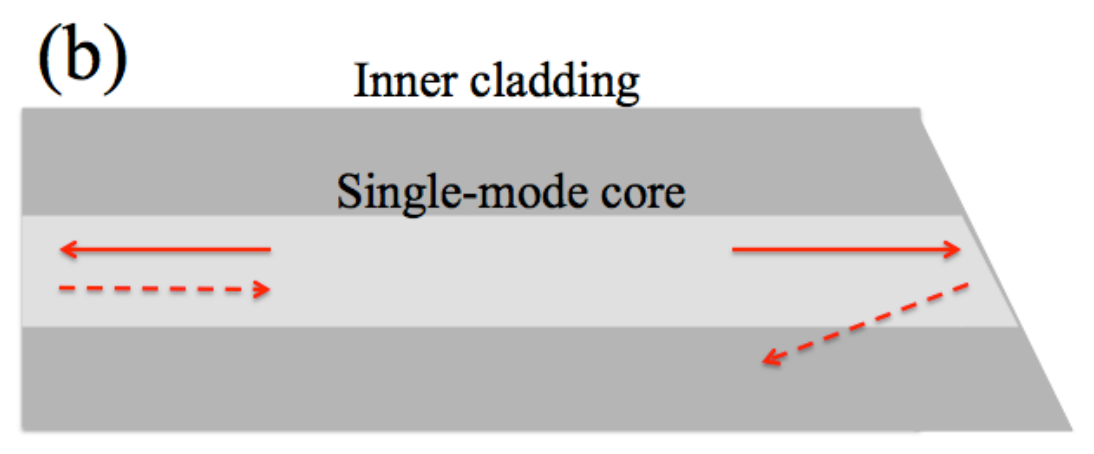

Figure B.2: Schematic diagram of cladding-pumping. (a) Pump light propagates through multi-mode inner cladding waveguide and is absorbed by $\mathrm{Yb}$ ions upon contact increasing population inversion. (b) Light reflection in double-clad fiber at a perpendicular fiber termination and at an angled fiber termination. Incident light is in solid red and reflected light dotted red.

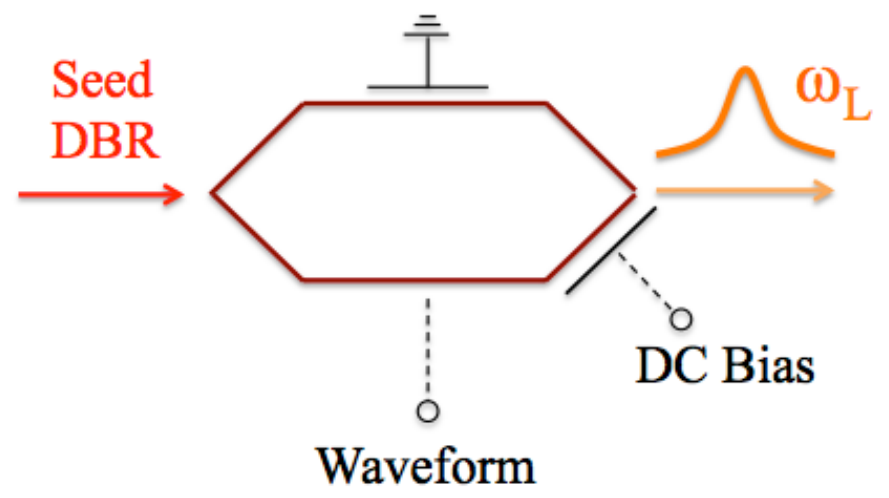

Figure B.3: Schematic diagram of a Mach-Zehnder modulator. Seed DBR laser light (red) enters modulator, the waveguide splits into to two paths and recombined onto an output waveguide generating intensity modulation at output. Generated waveguide (orange) driven by arbitrary function generator and DC bias voltage at quadrature point. 


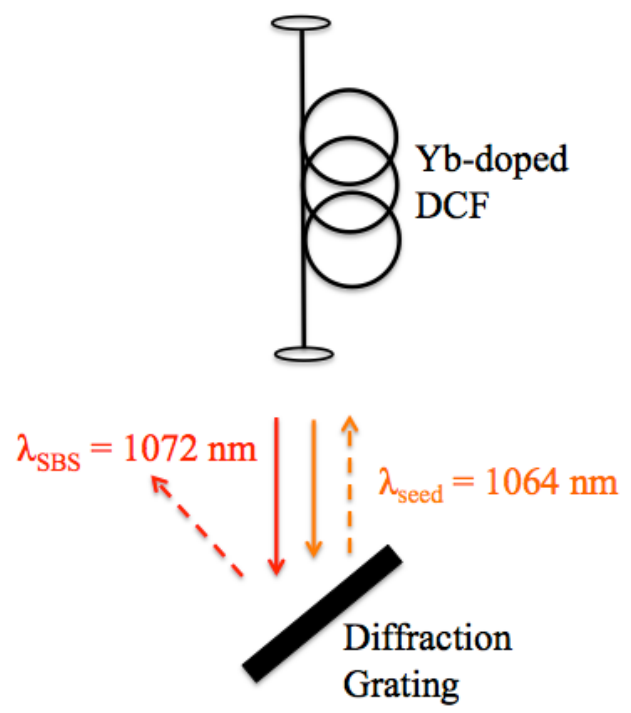

Figure B.4: Schematic diagram of the grating in the Littrow configuration. Free-running lasing mode at $1072 \mathrm{~nm}$ (red) are incident on the grating and the reflection (dotted red) are scattered and not spectrally selected to propagate back into the active fiber. The seeded mode at $1064 \mathrm{~nm}$ (orange) is reflected back off of the grating and tuned to propagate back into the fiber (dotted orange).

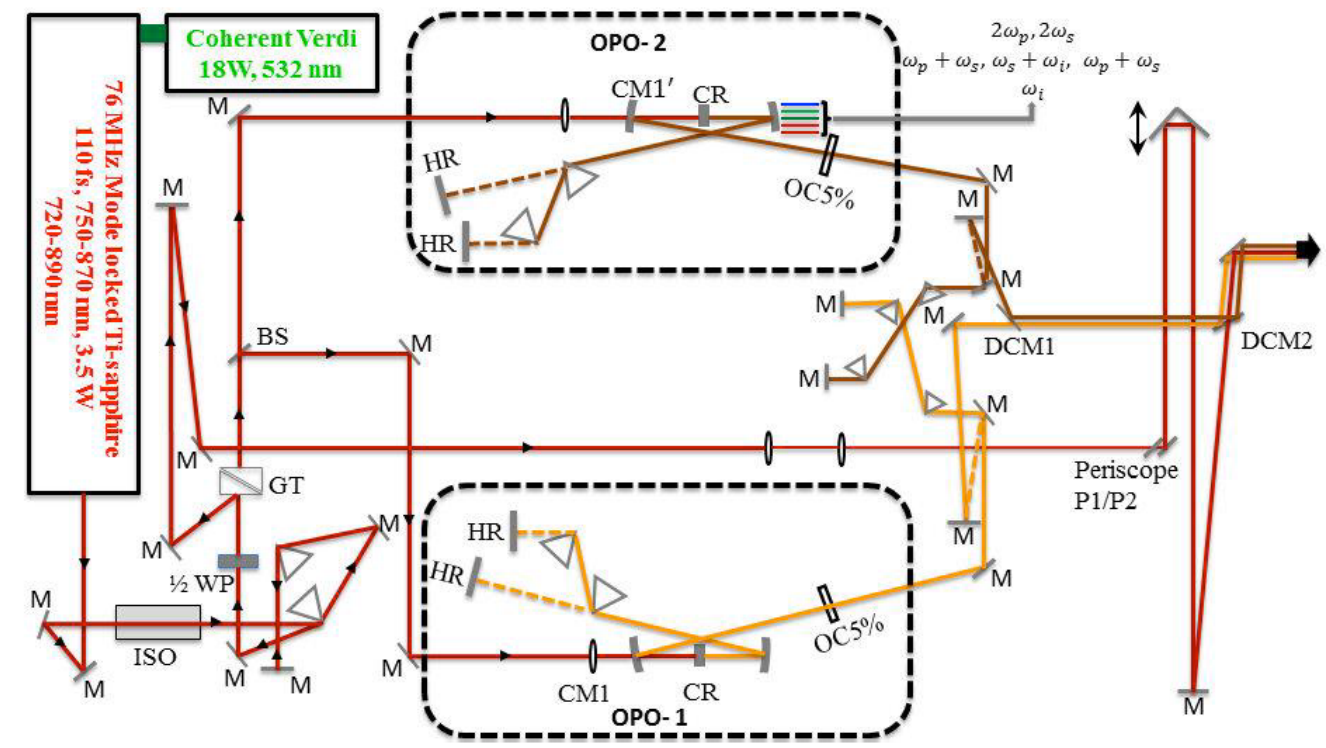


Figure B.5: Schematic of experimental CARS setup. ISO: isolator; WP: wave plate; GT: Glan-Thompson polarizer; BS: beamsplitter; HR: high reflector mirror; M: mirror; CR: crystal; OC: output coupler; DCM: dichroic mirror.

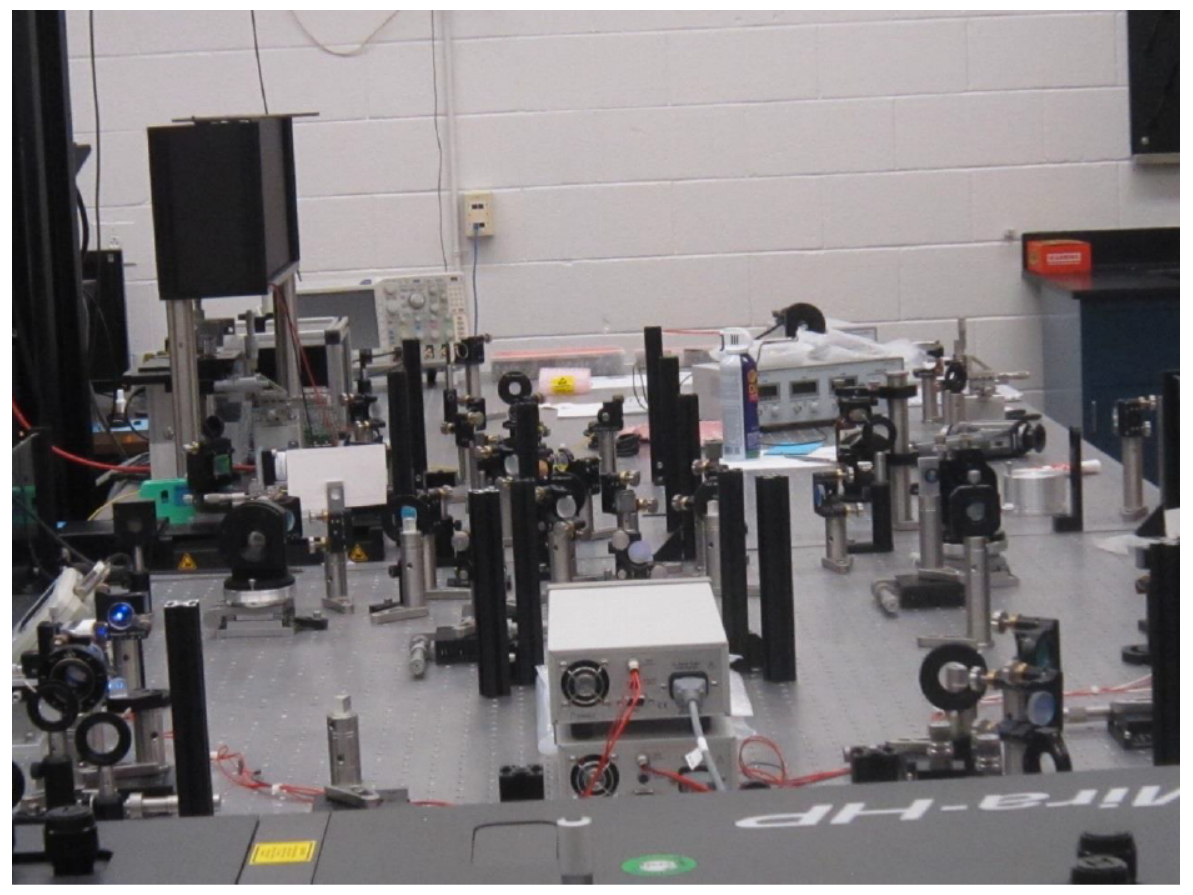

Figure B.6: Image of actual CARS experimental setup. 


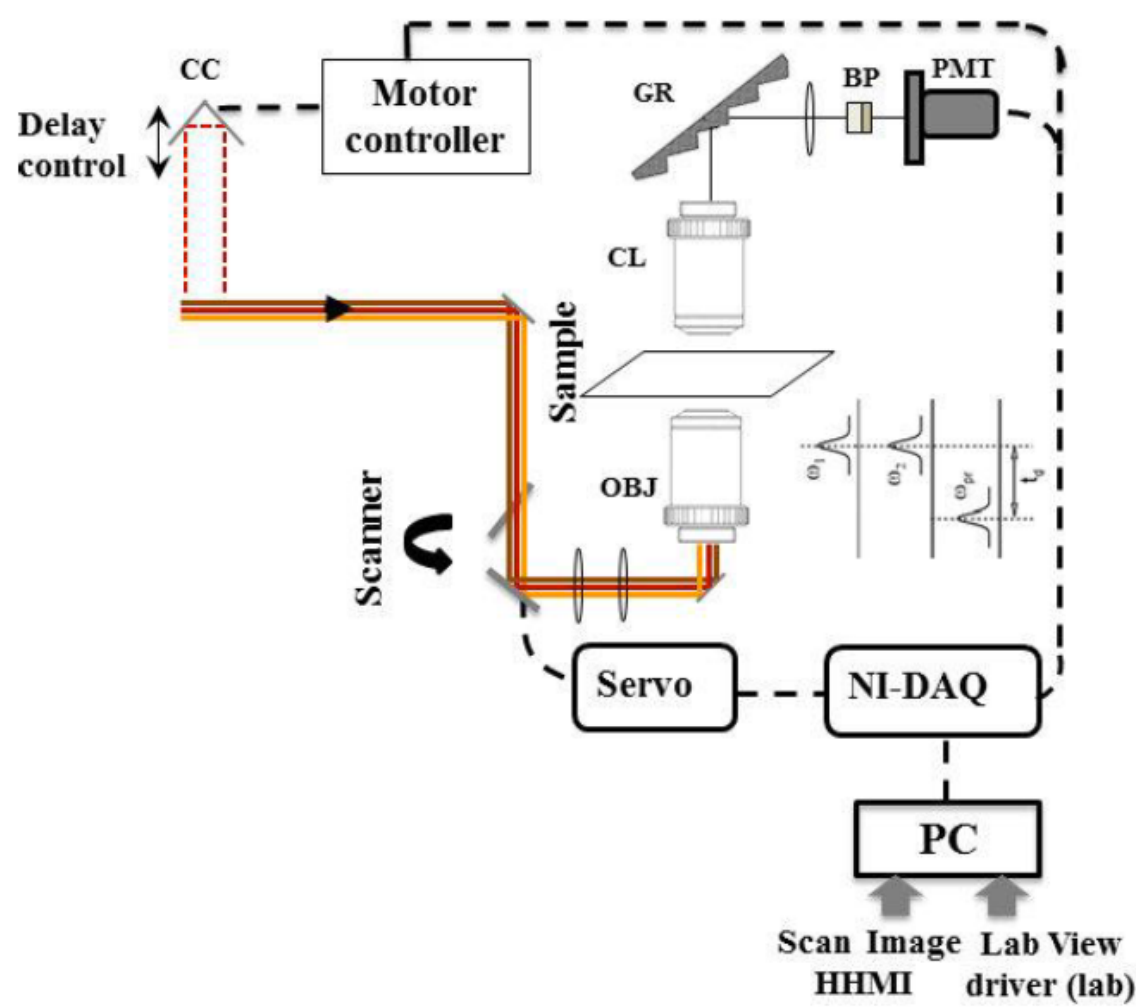

Figure B.7: Schematic diagram of image/data acquisition system. GR: grating; OBJ: objective; CC: corner cube; CL: collimating lens; BP: bandpass filter

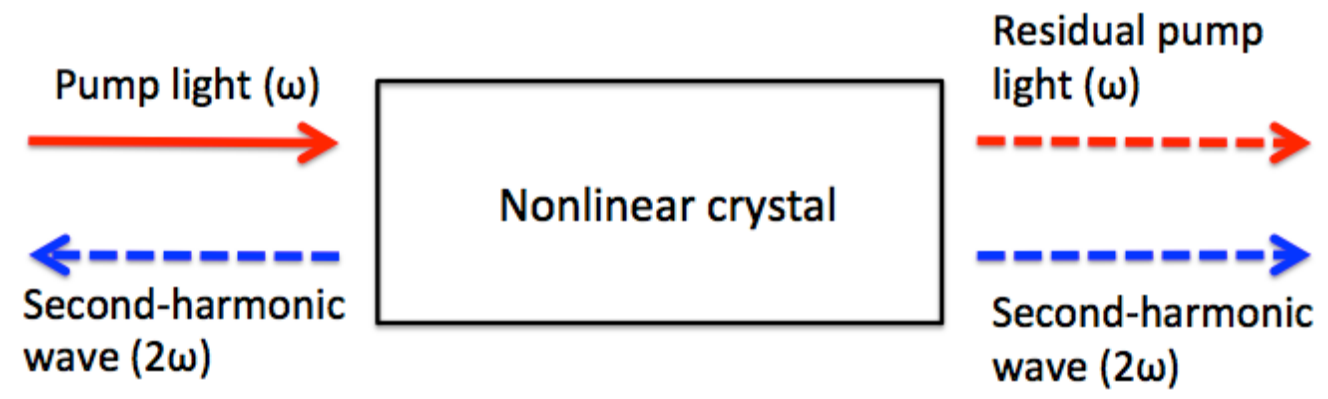

Figure B.8: Schematic diagram of a SHG process. An incident pump pulse (solid red) with optical frequency $\omega$ passes through a nonlinear crystal generating forward- and 
backward-propagating light (dotted blue) with frequency doubled $2 \omega$ and residual pump light (dotted red). 


\section{BIBLIOGRAPHY}

S. V. Chernikov, Y. Zhu, and J.R. Taylor, "Supercontinuum self-Q-switched ytterbium fiber laser," Opt. Lett. 22, 298-300 (1997).

E. Garmire, "Perspectives on stimulated Brillouin scattering," New Journal of Physics 19, 011003 (2017).

Y.O. Barmenkov, A.V. Kir'yanov, and M.V. Andres, "Nonlinear dynamics of Ytterbiumdoped fiber laser Q-switched using acousto-optical modulator,” The Eur. Phys. J. Special Topics 223, 2775-2788 (2014).

M. Laroche, H. Gilles, S. Girard, N. Passilly, and K. Ait-Ameur, “IEEE Photonics Technol. Lett. 18, 764-766 (2006).

L. Pan, I. Utkin, R. Lan, Y. Godwal, and R. Fedosejevs, "High-peak-power subnanosecond passively Q-switched ytterbium-doped fiber laser,” Opt. Lett. 35, 895897 (2010).

Z.J. Chen, A.B. Grudinin, J. Porta and J.D. Minelly, "Enhanced Q switching in doubleclad fiber lasers," Opt. Lett. 23, 454-456 (1998). 
Y. Fan, F. Lu, S. Hu, K. Lu, H. Wang, X. DOng, and G. Zhang, "105-kW Peak-Power Double-Clad Fiber Laser,” IEEE Photonics Technol. Lett. 15, 652-654 (2003).

M. Salhi, A. Hideur, T. Chartier, M. Brunel, G. Martel, and C. Ozkul, "Evidence of Brillouin scattering in an ytterbium-doped double-clad fiber laser," Opt. Lett. 27, 12941296 (2002).

A. Hideur, T. Chartier, M. Brunel, M. Salhi, C. Ozkul, and F. Sanchez, "Mode-lock, Qswitch and CW operation of an Yb-doped double-clad fiber ring laser,” Opti. Comm. 198, 141-146 (2001).

Y. Fan, F. Lu, S. Hu, K. Lu, H. Wang, and G. Zhang, "Narrow-linewidth widely tunable hybrid Q-switched double-clad fiber laser,” Opti. Lett. 28, 537-539 (2003).

B. Ortac, A. Hideur, T. Chartier, M. Brunel, G. Martel, M. Salhi, F. Sanchez, "Influence of cavity losses on stimulated Brillouin scattering in a self-pulsing side-pumped ytterbium-doped double-clad fiber laser," Opti. Comm. 215, 389-395 (2003).

R. W. Boyd, and K. Rzazewski, "Noise initiation of stimulated Brillouin scattering," Phys. Rev. A 42, 5514-5521 (1990).

A. L. Gaeta, and R. W. Boyd, "Stochastic dynamics of stimulated Brillouin scattering in an optical fiber,” Phys. Rev. A 44, 3205-3209 (1991). 
Z. Pan, L. Meng, Q. Ye, H. Cai, Z. Fang, and R. Qu, "Repetition rate stabilization of the SBS Q-switched fiber laser by external injection,” Opti. Expr. 17, 3124-3129 (2009).

L. Pan, I. Utkin, and R. Fedosejevs, "Experiment and Numerical Modeling of HighPower Passively Q-Switched Ytterbium-Doped Double-Clad Fiber Lasers,” IEEE Jour. of Quan. Elect. 46, 68-75 (2010).

B. N. Upadhyaya, A. Kuruvilla, U. Chakravarty, M. R. Shenoy, K. Thyagarajan, and S. M. Oak, "Effect of laser linewidth and fiber length on self-pulsing dynamics and output stabilization of single-mode Yb-doped double-clad fiber laser,” Appl. Opti. 49, 23162325 (2010).

Y. Tang, X. Li, and Q. J. Wang, "High-power passively Q-switched thulium fiber laser with distributed stimulated Brillouin scattering," Opti. Lett. 38, 5474-5477 (2013).

A. A. Fotiadi, P. Megret, and Michel Blondel, "Dynamics of a self-Q-switched fiber laser with a Rayleigh-stimulated Brillouin scattering ring mirror,” Opti. Lett. 29, 1078-1080 (2004).

Y. Wang, A. Martinez-Rios, and Hong Po, "Experimental study of stimulated Brillouin and Raman scatterings in a Q-switched cladding-pumped fiber laser," Opti. Fiber Tech. $10,201-214$ (2004). 
G. L. Keaton, M. J. Leonardo, M. W. Byer, and D. J. Richard, "Stimulated Brillouin scattering of pulses in optical fibers," Opti. Expr. 13351-13365 (2014).

Andrey Kobyakov, Michael Sauer, and Dipak Chowdhury, "Stimulated Brillouin scattering in optical fibers," Advances in Optics and Photonics 2, 1-59 (2010).

C. Ye, P. Yan, L. Huang, Q. Liu, and M. Gong, "Stimulated Brillouin scattering phenomena in a nanosecond linearly polarized Yb-doped double-clad fiber amplifier," Laser Phys. Lett 4, 376-381 (2007).

Boyd, Robert W. Nonlinear Optics. San Diego: Academic Press, 2003. Print.

X. D. Wang, P. Basseras, R.J.D. Miller, H. Vanherzelle, Appl. Phys. Lett. 59, 519 (1991).

Savatinova, I. Savova, E. Liarokapis, C.C. Ziling, V.V. Atuchin, M.N. Armenisek, V.M.N. Passrok, J. Phys.D: Appl. Phys. 31, 1667 (1998).

V. Pasiskevicius, A.Fragemann, F. Laurell, R. Butkus, V. Smilgevicius, A. Piskarskas, App. Phys. Lett. 82, 325 (2003).

V. Pasiskevicius, C. Canalias, F. Laurell, , App. Phys. Lett. 88, 041110 (2006). 
Y.F. Chen, Opt. Lett. 30,400 (2005).

L.L.Losev, J. Song, J.Xia, D. Strickland, and V. Brukhanov, Opt. Lett. 27, 2100 (2002).

M. Zhi, A. Sokolov, Opt. Lett. 32 , 2251 (2007).

E. Matsubara, T. Sekikawa, and M. Yamashita, App. Phys. Lett. 92, 071104 (2008).

S. Baker, I. Walmsley, J.W.G. Tisch, and J.P.Marangos, Nature Photonics 5, 664 (2011).

G. E. Kugel, F. Brehatt, B. Wyncket, M. D. Fontanat, G. Marniers, C. CarabatosNedelect, J. Mangin, J. Phys. C: Solid State Phys. 21, 5565 (1988).

G.H. Watson, J. Raman Spectrosc. 22, 705 (1991).

K. Vivekanandan, S. Setvasekarapandian, P. Kolandaivel, M.T. Sebastian, S. Suma, Mater. Chem. Phys. 49, 204 (1997).

G.A Massey, T.M. Loehr, L.J. Willis, J.C. Johnson, Appl. Optics 19, 4136 (1980).

V.M Garmash, D.N. Govorun, P.A. Korotkov, V.V Obukhovskii, N.I. Pavlova, I.S Rez, Opt. Spectrosc. 58, 424 (1985). 
G.A. Kourouklis, A. Jayaraman, A.A. Ballamn, Solid State Commun. 62, 379 (1987).

Y.K. Voronki. V.A. Dyahov, A.B. Kudryavtsev, V.V. Osiko, A.A. Sobol, E.V. Sorokin,Sov.Phys.-Solid State 31, 1736 (1989).

S.Fursawa, H. Hayasi, Y. Ishibashi, A. Miyamoto, T.Sasaki, J.Phys.Soc.Jpn. 60, 2470 (1991).

C.S. Tu, A.R. Guo, R. Tao, R.S. Katiyar, R. Guo, A.S. Bhalla, J. Appl. Phys. 79, 3235 (1996).

S.Yang, R.Wysolmerski, and F. Ganikhanov, Opt. Lett. 36, 3849 (2011).

S.Yang, S.Adhikari, L. Zhang, R.Wysolmerski, G, Spirou, and F. Ganikhanov, Appl. Phys B: Lasers and Optics 111, 617 (2013).

K.Bhupathiraju, A.Seymour, F.Ganikhanov, Opt. Lett. 34, 2092 (2009).

J.Rowley, S.Yang, F. Ganikhanov, J. Opt. Soc. Am. B 28, 1026 (2011).

A. Laubereau, D. von der Linde, and W. Kaiser, Phys. Rev. Lett. 28, 1162 (1972). 
A. Laubereau and W. Kaiser, Rev. Mod. Phys. 50, 607 (1978).

S. A. Malinovskaya, Opt. Lett. 33, 2245(2008).

A.Jerry, Introduction to Integral Equations With Applications, (2d edition, John Willey and Sons, Inc.,1999).

S. Yang, F. Ganikhanov, Opt. Lett. 38, 4754 (2013).

Ch. Ferrer, A. Segura, M.V. Andres, V. Munoz, J. Pellicer, J. App. Phys. 79,3200 (1996).

X. Liwen, C. Dawei, N. Hongda, Chinese Phys. Lett. 1.6,225 (1989).

M.J. Bushiri, V.P. M. Pillai, R. Ratheesh, V.U. Nayar, J. Phys. Chem. Solids 60, 1983 (1999).

M.J. Bushiri, V.U. Nayar, J. Nonlinear Optic. Phys. Mat. 10, 345 (2001).

Qu, K. ; Barth, I. ; Fisch, N.J. Plasma wave seed for Raman amplifiers. Phys. Rev. Lett. 2017, 118, 164801-6. 
Jin, H. ; Liu, F.M. ; Xu, P. ; Xia, J.L. ; Zhong, M.L. ; Yuan, Y. ' ; Zhou, J.W. ; Gong, Y.X. ; Wang, W. ; Zhu, S.N. On-chip Generation and Manipulation of Entangled Photons Based on Reconfigurable Lithium-niobate Waveguide Circuits. Phys. Rev. Lett. 2014, 113, 103601-5.

Bhaskar, M.K. ; Sukachev, D.D. ; Sipahigil, A. ; Evans, R.E. ; Burek, M.J. ; Nguyen, C.T. ; Rogers, L.J. ; Siyushev, P. ; Metsch, M.H. ;Park, H. ; Jelezko, F. ; Lonc`ar, M. ; Lukin, M.D. Quantum nonlinear optics with a germanium-vacancy color center in a nanoscale diamond waveguide. Phys. Rev. Lett. 2017, 118, 223603-6.

Nguyen, H.A. ; Grange, T. ; Reznychenko, B. ; Yeo, I. ; de Assis, P.-L. ; Tumanov, D. ; Fratini, F. ; Malik, N.S. ; Dupuy, E. ; Gregersen, N. ; Auffe'ves, A. ; Ge'rard, J.M. ; Claudon, J. ; Poizat, J.-P. Giant nonlinear interaction between two optical beams via a quantum dot embedded in a photonic wire. Phys. Rev. B. 2018, 97, $201106-5$.

Cicchi, R. ; Mattha“us, C. ; Meyer, T. ; Lattermann, A. ; Dietzek, B. ; Brehm, B.R. ; Popp, J. ; Pavone, F.S. Characterization of collagen and cholesterol deposition in atherosclerotic arterial tissue using non-linear microscopy. J. Biophotonics 2014, 7, 13543.

Denk, W.; Strickler, J.H.; Webb, W.W. Two-photon laser scanning microscopy. Science 1990, 248, 73-76. 
Campagnola, P.J.; Millard, A.C.; Terasaki, M.; Hoppe, P.E.; Malone, C.J.; Mohler, W.A. Three-dimensional high-resolution second-harmonic generation imaging of endogenous structural proteins in biological tissues. Biophys. J. 2002, 82, 493-508.

Débarre, D.; Suppato, W.; Pena, A.M.; Fabre, A.; Tordjmann, T.; Combettes, L.; Schanne-Klein, M.-C.; Beaurepaire, E. Imaging lipid bodies in cells and tissues using third-harmonic generation microscopy. Nat. Meth. 2006, 3, 47-53.

Evans, C.L.; Potma, E.O.; Puoris'haag, M.; Coté, D.; Lin, C.P.; Xie, X.S. Chemical imaging of tissue in vivo with video-rate coherent anti-Stokes Raman scattering microscopy. Proc. Natl. Acad. Sci. USA 2005, 102, 16807-16812.

Freudiger, C.W.; Min, W.; Saar, B.G.; Lu, S.; Holtom, G.R.; He, C.; Tsai, J.C.; Kang, J.X.; Xie, X.S. Label-free biomedical imaging with high sensitivity by stimulated Raman scattering microscopy. Science 2008, 322, 1857.

Widulle, F.; Ruf, T.; Schonherr, E.; Cardona, M. Investigation of the anomalous Raman line shape of the TO phonon in GaP. Phys. Status Solidi B 1999, 215, 131-6.

Joshi, J. ; Stone, I.R.; Beams, R.; Krylyuk, S.; Kalish, I.; Davydov, A.V.; Vora, P.M. Phonon anharmonicity in bulk $\mathrm{T}_{\mathrm{d}}-\mathrm{MoTe}_{2}$. Appl. Phys. Lett. 2016,109, 031903-5. 
Kaminskii, A.A. ; Lux, O.; Rhee, H.; Eichler, H.J.; Yoneda, H.; Shirakawa, A.; Ueda, K.; Zhao, B.; Chen, J.; Dong, J.;Zhang, J. Crystal-host $\mathrm{Gd}_{0.5} \mathrm{Lu}_{0.5} \mathrm{VO}_{4}$ for $\mathrm{Ln}^{3+}$-lasants: a new high-gain many-phonon $\chi^{(3)}$-active tetragonal vanadate-SRS spectroscopy and nonlinear-laser effects. Appl. Phys. B 2012,109, 649-58.

Kaminskii, A.A. ; Lux, O.; Rhee, H.; Kaltenbach, A.; Eichler, H.J.; Zhang, J.; Tang, D.Y.; Yu, H.; Zhang, H.; Wang, J.;Yoneda, H.; Ueda, K.; Shirakawa, A. Lowtemperature stimulated Raman scattering spectroscopy of tetragonal $\mathrm{GdVO}_{4}$ single crystals. Phys. Status Solidi B 2014, 251, 1045-62.

Liu, W.; Wang, H.; Du, J; Jing, C. Raman microspectroscopy of nucleus and cytoplasm for human colon cancer diagnosis. Biosens. Bioelectron 2017, 97, 70.

Eberhardt, K.; Beleites, C.; Marthandan, S.; Matth€aus, C.; Diekmann, S.; Popp, J. Raman and Infrared Spectroscopy Distinguishing Replicative Senescent from Proliferating Primary Human Fibroblast Cells by Detecting Spectral Differences Mainly Due to Biomolecular Alterations. Anal. Chem. 2017, 89, 2937.

Thomas, G.J. Raman spectroscopy of protein and nucleic acid assemblies. Annu. Rev. Biophys. Biomol. Struct. 1999, 28, 1. 
Korifi, R.; Le Dreau, Y.; Molinet, J.; Artaud, J.; Dupuy, N. Composition and authentication of virgin olive oil from French PDO regions by chemometric treatment of Raman spectra. J. Raman Spectrosc. 2011, 42, 1540.

Potma, E.O.; Evans, C.L.; Xie, X.S. Heterodyne coherent anti-Stokes Raman scattering (CARS) imaging. Optics Letters 2006, 31(2), 241-243.

Saar, B.G.; Freudiger, C.W.; Reichman, J.; Stanley, C.M.; Holtom, G. R.; Xie, X.S. Video-Rate Molecular Imaging in Vivo with Stimulated Raman Scattering. Science 2010, 330(6009), 1368-1370.

Racu, A.-M.; Menzel, D.; Schoenes, J.; Doll, K. Crystallographic disorder and electronphonon coupling in $\mathrm{Fe}_{1-\mathrm{x}} \mathrm{Co}_{\mathrm{x}} \mathrm{Si}$ single crystals: Raman spectroscopy study. Phys. Rev. B. 2007, 76, 115103-7.

Giarola, M. ; Sanson, A.; Rahman, A.; Mariotto, G. ; Bettinelli, M. ; Speghini, A. ; Cazzanelli, E. Vibrational dynamics of $\mathrm{YPO}_{4}$ and $\mathrm{ScPO}_{4}$ single crystals: An integrated study by polarized Raman spectroscopy and first-principles calculations. Phys. Rev. B. 2011, 83, 224302-8.

Pavillon, N. ; Hobro, A.J.; Smith, N.I. Cell optical density and molecular composition revealed by simultaneous multimodal label-free imaging. Biophys. J. 2013, 105, 1123-32. 
Corsetti, S. ; Rabl, T.; McGloin, D.; Nabi, G. Raman spectroscopy for accurately characterizing biomolecular changes in androgen-independent prostate cancer cells. $J$. Biophotonics 2018, 11, 1-8.

Giordmaine, J. A.; Kaiser, W. Light scattering by coherently driven lattice vibrations. Physical Review 1966, 144(2), 676.

Laubereau, A.; Kaiser, W. Vibrational dynamics of liquids and solids investigated by picosecond light pulses. Reviews of Modern Physics 1978, 50(3), 607.

Jerry, A. Introduction to Integral Equations With Applications, $2^{\text {nd }}$ ed.; John Wiley \& Sons, Inc., 1999.

Malinovskaya, S.A. Prevention of decoherence by two femtosecond chirped pulse trains. Optics letters 2008, 33(19), 2245-2247.

Rowley, J.; Yang, S.; Ganikhanov, F. Power and tuning characteristics of a broadly tunable femtosecond optical parametric oscillator based on periodically-poled stoichiometric lithium tantalate. JOSA B 2011, 28, 1026-1036.

Yang, S.; Adhikari, S.; Dobbala, M.; Adusumilli, S.; Rowley, J.; Zhang, L.; Marrs, G.; Wysolmerski, R.; Spirou, G.; Ganikhanov, F. Multi-color ultrafast laser platform for 
nonlinear optical imaging based on independently tunable optical parametric oscillators. Appl. Phys. B: Lasers and Optics 2013, 111, 617-625.

Kugel, G. E.; Brehat, F.; Wyncke, B.; Fontana, M. D.; Marnier, G.; Carabatos-Nedelec, C.; Mangin, J. The vibrational spectrum of a KTiOPO4 single crystal studied by Raman and infrared reflectivity spectroscopy. Journal of Physics C: Solid State Physics $\mathbf{1 9 8 8 ,}$ 21(32), 5565 .

Vivekanandan, K.; Selvasekarapandian, S.; Kolandaivel, P.; Sebastian, M. T.; Suma, S. Raman and FT-IR spectroscopic characterisation of flux grown KTiOPO4 and KRbTiOPO4 non-linear optical crystals. Materials chemistry and physics 1997, 49(3), 204-210.

Watson, G. H. Polarized Raman spectra of ktioaso4 and isomorphic nonlinear-optical crystals. J. Raman Spectrosc. 1991, 22(11), 705-713.

D'yakov, Y. E. Dephasing in steady-state and time-varying spectroscopy. JETP Lett. $1983,37,15$.

El-Abassy, R.M.; Donfack, P.; Materny, A. Visible Raman spectroscopy for the discrimination of olive oils from different vegetable oils and the detection of adulteration. J. Raman Spectrosc 2009, 40, 1284. 
B. Radisavljevic, A. Radenovic, J. Brivio, V. Giacometti, and A. Kis, "Single-layer $\operatorname{MoS}_{2}$ Transistors," Nat. Nanotechnol. 6, 147-150 (2011).

N. Kumar, S. Najmaei, Q. Cui, F. Ceballos, P. M. Ajayan, J. Lou, and H. Zhao, "Second harmonic microscopy of monolayer $\mathrm{MoS}_{2}, "$ Phys. Rev. B 87, 161403-5 (2013).

L. M. Malard, T. V. Alencar, A.P.M. Barboza, K. F. Mak, and A.M. de Paula, "Observation of intense second harmonic generation from $\mathrm{MoS}_{2}$ atomic crystals," Phys. Rev. B 87, 201401-5 (2013).

E. Mishina, N. Sherstyuk, S. Lavrov, A. Sigov, A. Mitioglu,S. Anghel, and L. Kulyuk, "Observation of two polytypes of $\mathrm{MoS}_{2}$ ultrathin layers studied by second harmonic generation microscopy and photoluminescence," Appl. Phys. Lett. 106, 31901-5 (2015).

G. Wang, X. Marie, I. Gerber, T. Amand, D. Lagarde, L. Bouet, M. Vidal, A. Balocchi, and B. Urbaszek, "Giant Enhancement of the Optical Second-Harmonic Emission of $\mathrm{WSe}_{2}$ Monolayers by Laser Excitation at Exciton Resonances,"Phys. Rev. Lett. 114, 097403-6 (2015).

A. Ramasubramaniam, "Large excitonic effects in monolayers of molybdenum and tungsten dichalcogenides," Phys. Rev. B 86, 115409-6 (2012). 
V. G. Dmitriev, G. G. Gurzadyan, Handbook of Nonlinear Optical Crystals (Springer Series in Optical Sciences), 2d Edition, (1996).

N. Bloembergen, P.S. Pershan , "Light Waves at the Boundary of Nonlinear Media ," Phys. Rev. 128, 606-622 (1962).

M. Merano, "Nonlinear Optical Response of a Two-dimensional atomic crystal," Opt. Lett. 41,187-190 (2016).

M. Merano, "Fresnel coefficients of a two-dimensional atomic crystal," Phy. Rev. A 93, $013832(2016)$.

G.D. Boyd, D.A.Kleinman, "Parametric interaction of Focused gaussinan light beams ," J. Appl. Phys. 39, 3597-3639 (1968).

Y.Li, Y.Rao, K.F.Mak, Y.You, S.Wang, C.R.Dean, and T.F.Heinz,“ Probing symmetry properties of Few-layer $\mathrm{MoS}_{2}$ and h-BN by optical second-harmonic generation," Nano Lett. 13, 3329-3333 (2013).

S.M. Eichfeld, C.M.Eichfeld, Y-C. Lin, L. Hossain. J.A.Robinson, "Rapid, nondestructive evaluation of ultrathin WSe2 using spectroscopic ellipsometry," APL Mater. 2, 092508 (2014). 
I. Shoji, T. Kondo, A. Kitamoto, M. Shirane, and R. Ito, "Absolute scale of second-order nonlinear-optical coefficients,’J. Opt. Soc. Am. 14, 2268-2294 (1997).

K. Vodopyanov, O. Levi, P.Kuo, T. Pinguet, J.Harris, M.Fejer, B. Gerard, L. Becouarn, and E. Lallier, "Optical parametric oscillation in quasi-phase-matched GaAs" Opt. Lett. 29, 1912-1914 (2004).

M. L. Trolle, G. Seifert, T. G. Pedersen,"Theory of excitonic second harmonic generation in monolyaer $\mathrm{MoS}_{2}, "$ Phys. Rev B 89, 235410 (2014).

R. Leitsmann, W.G.Schmidt, P.H.Hahn, and F.Bechstedt, "Second-harmonic polarizability including electron-hole attraction from band-structure theory,” Phys. Rev. B 71, 195209 (2005). 